\title{
Stellar populations of galaxies in the ALHAMBRA survey up to $\mathrm{z} \sim 1$ \\ II. Stellar content of quiescent galaxies within the dust-corrected stellar mass-colour and the UVJ colour-colour diagrams ${ }^{\star, \star \star}$
}

\author{
L. A. Díaz-García ${ }^{1,2}$, A. J. Cenarro ${ }^{3}$, C. López-Sanjuan ${ }^{3}$, I. Ferreras ${ }^{4}$, M. Cerviño ${ }^{5,6}$, A. Fernández-Soto ${ }^{7,8}$, \\ R. M. González Delgado ${ }^{5}$, I. Márquez ${ }^{5}$, M. Pović ${ }^{5}$, I. San Roman ${ }^{1}$, K. Viironen ${ }^{3}$, M. Moles ${ }^{1}$, D. Cristóbal-Hornillos ${ }^{3}$, \\ A. López-Comazzi ${ }^{1}$, E. Alfaro ${ }^{5}$, T. Aparicio-Villegas ${ }^{9}$, N. Benítez ${ }^{5}$, T. Broadhurst ${ }^{10,11}$, J. Cabrera-Caño ${ }^{12}$, \\ F. J. Castander ${ }^{13}$, J. Cepa ${ }^{6,14}$, C. Husillos ${ }^{5}$, L. Infante ${ }^{15,16}$, J. A. L. Aguerri ${ }^{6,14}$, V. J. Martínez ${ }^{17,18,8}$, J. Masegosa ${ }^{5}$, \\ A. Molino ${ }^{19}$, A. del $\mathrm{Olmo}^{5}$, J. Perea ${ }^{5}$, F. Prada ${ }^{5}$, and J. M. Quintana ${ }^{5}$
}

(Affiliations can be found after the references)

Received 7 February 2018 / Accepted 27 August 2019

\begin{abstract}
Aims. Our aim is to determine the distribution of stellar population parameters (extinction, age, metallicity, and star formation rates) of quiescent galaxies within the rest-frame stellar mass-colour diagrams and $U V J$ colour-colour diagrams corrected for extinction up to $z \sim 1$. These novel diagrams reduce the contamination in samples of quiescent galaxies owing to dust-reddened galaxies, and they provide useful constraints on stellar population parameters only using rest-frame colours and/or stellar mass.

Methods. We set constraints on the stellar population parameters of quiescent galaxies combining the ALHAMBRA multi-filter photo-spectra with our fitting code for spectral energy distribution, MUlti-Filter FITting (MUFFIT), making use of composite stellar population models based on two independent sets of simple stellar population (SSP) models. The extinction obtained by MUFFIT allowed us to remove dusty star-forming (DSF) galaxies from the sample of red $U V J$ galaxies. The distributions of stellar population parameters across these rest-frame diagrams are revealed after the dust correction and are fitted by LOESS, a bi-dimensional and locally weighted regression method, to reduce uncertainty effects.

Results. Quiescent galaxy samples defined via classical $U V J$ diagrams are typically contaminated by a $\sim 20 \%$ fraction of DSF galaxies. A significant part of the galaxies in the green valley are actually obscured star-forming galaxies $(\sim 30-65 \%)$. Consequently, the transition of galaxies from the blue cloud to the red sequence, and hence the related mechanisms for quenching, seems to be much more efficient and faster than previously reported. The rest-frame stellar mass-colour and $U V J$ colour-colour diagrams are useful for constraining the age, metallicity, extinction, and star formation rate of quiescent galaxies by only their redshift, rest-frame colours, and/or stellar mass. Dust correction plays an important role in understanding how quiescent galaxies are distributed in these diagrams and is key to performing a pure selection of quiescent galaxies via intrinsic colours.
\end{abstract}

Key words. galaxies: stellar content - galaxies: photometry - galaxies: evolution - galaxies: formation

\section{Introduction}

Galaxies exhibit a well-known bimodal distribution of colours; they are usually referred to as red and blue galaxies, with welldifferentiated properties (e.g. Bell et al. 2004; Baldry et al. 2004; Williams et al. 2009; Ilbert et al. 2010; Peng et al. 2010; Arnouts et al. 2013; Moresco et al. 2013; Pović et al. 2013; Fritz et al. 2014; Schawinski et al. 2014). Red galaxies present more evolved stellar populations with lower star formation levels, and so are usually called passive or quiescent. In contrast, blue galaxies exhibit younger stellar populations, typically with signatures of strong star formation processes. Understanding the formation and evolution of the so-called quiescent or passive galaxies is still a challenge today; these galaxies started to form stars at very

^ Full Table 1 and the stellar population properties are only available at the CDS via anonymous ftp to cdsarc.u-strasbg.fr (130.79.128.5) or via http://cdsarc.u-strasbg.fr/viz-bin/ cat/J/A+A/631/A156

$\star \star$ Based on observations collected at the Centro Astronómico Hispano Alemán (CAHA) at Calar Alto, operated jointly by the MaxPlanck Institut für Astronomie and the Instituto de Astrofísica de Andalucía (CSIC). early epochs, to later shut down their star formation by mechanisms that are a matter of debate even today (see references in Peng et al. 2015).

One of the most extended diagrams for disentangling different types of galaxies involves rest-frame colours and absolute magnitudes (e.g. Bell et al. 2004; Baldry et al. 2004; Brown et al. 2007). These diagrams, usually referred to as colour-magnitude diagrams (CMDs), clearly show the bimodal distribution of galaxies previously mentioned. Within this diagram, galaxies are usually referred to as red-sequence galaxies (predominantly old and including very massive galaxies in the nearby Universe) and blue cloud galaxies (numerous and with a prominent on-going star formation). In addition, some authors have proposed that there is a third group of galaxies lying in the colour region between these two dominant groups of galaxies, which we call the green valley. Interestingly, the presence of active galactic nuclei (AGNs) in green valley galaxies is remarkable (Nandra et al. 2007; Bundy et al. 2008; Georgakakis et al. 2008; Silverman et al. 2008; Hickox et al. 2009; Schawinski et al. 2009; Pović et al. 2012). Owing to this overdensity of galaxies hosting an AGN in the green valley, some authors have proposed that the presence of AGNs may be related to a quenching 
mechanism that produces a transition of galaxies from the blue cloud to the red sequence (e.g. Faber et al. 2007; Schawinski et al. 2007). However, there is also evidence to show that galaxies belonging to the green valley are mainly dust-reddened starforming (hereafter DSF) galaxies (Bell et al. 2005; Cowie \& Barger 2008; Brammer et al. 2009; Cardamone et al. 2010).

During the last decade, the rest-frame colour-colour diagrams, especially the $U V J$ diagram, have become one of the most extended methods used to select quiescent and star-forming galaxies (e.g. Daddi et al. 2004; Williams et al. 2009; Arnouts et al. 2013). The advantage of these diagrams is that they use two colours, one in the blue part of the spectral energy distribution (SED), $U-V$ or NUV $-r$, the other in the red part, $V-J$ or $r-K$; the two colours reduce the contamination of DSF galaxies in the quiescent sample with predominant red colours and low star formation rates (e.g. Williams et al. 2009; Arnouts et al. 2013; Ilbert et al. 2013; Moresco et al. 2013). These colour-colour diagrams are efficient because the galaxies empirically present more separated loci between the red and blue populations, whereas in CMDs the two galaxy populations appear largely overlapped (Wyder et al. 2007; Cowie \& Barger 2008; Brammer et al. 2009). Even though $U V J$ diagrams have been demonstrated to be a reliable method for splitting quiescent from star-forming galaxies, these diagrams present a level of contamination in the selection of quiescent galaxies that depends on redshift and stellar mass (Williams et al. 2009; Moresco et al. 2013). In some cases the number of star-forming interlopers may reach $30 \%$ of galaxies at certain redshift and mass regimes, or at least $15 \%$ after imposing a more restrictive $U-V$ colour limit than the one defined originally (Moresco et al. 2013).

Alternative methods for selecting galaxies by spectral type comprise colours and stellar masses (Peng et al. 2010), but these are almost equivalent to CMDs because the absolute magnitudes or luminosities are tightly linked to stellar mass. Therefore, colour-stellar mass diagrams present similar and non-negligible contamination of DSF galaxies in the red part since they are not able to disentangle the reddening by dust. Other works (e.g. Noeske et al. 2007; Whitaker et al. 2012; Moustakas et al. 2013; Fumagalli et al. 2014) suggest introducing star formation rates (SFRs) along with stellar masses as a criterion to separate quiescent and star-forming galaxies. The disadvantage of this diagram is that we need a previous estimation of the mentioned stellar population parameters, which in many cases is not available or is hard to calculate. In some cases, SFRs and specific star formation rates (sSFRs, defined as the star formation rate per unit of stellar mass, i.e. SFR $/ M_{\star}$ ) can also be used as an additional (or the unique) criterion to select quiescent galaxies below a predefined threshold (e.g. Ilbert et al. 2010; Pozzetti et al. 2010; Domínguez Sánchez et al. 2011, 2016).

Recent studies show that some stellar population parameters are related to the range of colours that galaxies occupy in $U V J$ colour-colour diagrams (Belli et al. 2015; Domínguez Sánchez et al. 2016; Martis et al. 2016; Pacifici et al. 2016; Yano et al. 2016; Fang et al. 2018). There is a general agreement amongst the results, which indicates that certain ranges of SFR and SSFR values are well constrained in precise colour ranges within $U V J$ diagrams. This means that galaxies in the typical colour ranges enclosing quiescent galaxies exhibit low sSFR values, whereas the higher SSFR values lie on the lower parts of this kind of diagrams. There is evidence to support a continuous SSFR gradient perpendicular to the empirical colour limit separating quiescent from star-forming galaxies in $U V J$ diagrams (see e.g. Belli et al. 2015; Fang et al. 2018). In addition, and as expected from extinction laws, the most dust-reddened galaxies populate the reddest parts of these diagrams (see e.g. Martis et al. 2016; Fang et al. 2018). This suggests that rest-frame $U V J$ diagrams can be used as stellar population estimators to predict, or at least constrain, the stellar population properties of galaxies. However, this potential idea, especially for quiescent galaxies, has not been extensively exploited in detail yet. Usually stellar populations are only plotted on these diagrams with the unique purpose of a sanity check. Large-scale multi-filter surveys can provide a large number of galaxies to largely populate the full range of colours for any kind of rest-frame colour-colour diagrams at wide redshift ranges and stellar masses.

In this series of papers, our aim is to improve our knowledge of the evolution of quiescent galaxies since $z \sim 1$ through the use of spectrophotometric data from the Advanced Large Homogeneous Area Medium Band Redshift Astronomical survey (ALHAMBRA; Moles et al. 2008). In this second paper in the series we focus on the distribution of the stellar population parameters of quiescent galaxies within the $U V J$ diagram. For the first time, we dissected the loci of age, metallicity, extinction, and sSFR simultaneously for a complete and numerous sample of quiescent galaxies from the ALHAMBRA survey. In addition, we extended this study to the stellar mass-colour diagram, which illustrates the influence of stellar mass more clearly and complements the reliability around the selection of quiescent galaxies.

This paper is organised as follows. In Sect. 2 we briefly present the main features of the ALHAMBRA survey. In Sect. 3, we summarise the SED fitting methodology embedded in MUFFIT $^{1}$ and the set of simple stellar population (SSP) models used to constrain the stellar populations of galaxies. Section 4 details the process to build a reliable sample of quiescent galaxies, and to determine the stellar mass completeness of the sample. The techniques used to obtain SFRs, and therefore sSFRs, from the SED fitting results provided by MUFFIT are shown in Sect. 5. The main result of this work, which is the distribution of the stellar population parameters of quiescent galaxies within stellar mass-colour and colour-colour rest-frame diagrams, is given in Sect. 6. Implications from our results are discussed in Sect. 7. The conclusions of this work are summarised in Sect. 8.

Throughout this paper we adopted a Lambda cold dark matter $(\Lambda \mathrm{CDM})$ cosmology with $H_{0}=71 \mathrm{~km} \mathrm{~s}^{-1}, \Omega_{\mathrm{M}}=0.27$, and $\Omega_{\Lambda}=0.73$. All magnitudes are in the AB-system (Oke \& Gunn 1983). Stellar masses are quoted in solar mass units $\left[M_{\odot}\right]$. In this work, we assumed a Chabrier (2003) and Kroupa Universal (Kroupa 2001) initial stellar mass functions (IMF, more details in Sect. 3).

\section{Photometric data from the ALHAMBRA survey}

The ALHAMBRA survey ${ }^{2}$ provides flux in 23 photometric bands ${ }^{3}$ in AB-magnitudes (corrected for point spread function, PSF; Coe et al. 2006), 20 in the optical range $\lambda \lambda$ 3500-9700 $\AA$ (Aparicio Villegas et al. 2010, top hat medium-band filters, $\sim 300 \AA$ width, overlapping close to zero between contiguous bands) and 3 in the near-infrared (NIR) spectral window $\lambda \lambda 1.0$ $2.3 \mu \mathrm{m}$ ( $J, H$, and $K_{s}$ bands), for each source in seven noncontiguous fields along the northern hemisphere. The current effective area is $\sim 2.8 \mathrm{deg}^{2}$ and the total on-target exposure time is $\sim 700 \mathrm{~h}(\sim 608 \mathrm{~h}$ for bands in the optical range, and $\sim 92 \mathrm{~h}$ for the NIR). Magnitude limits for optical bands ( $5 \sigma$ level and $3^{\prime \prime}$

\footnotetext{
MUlti-Filter FITting for stellar population diagnostics.

http: //www . alhambrasurvey . com

http://svo2.cab.inta-csic.es/theory/fps3/
} 
aperture) range from $m_{\mathrm{AB}} \sim 24$ for the 14 bluer filters, decreasing towards the red, reaching $m_{\mathrm{AB}} \sim 22$ in the reddest one (Molino et al. 2014). For the NIR range, magnitude limits are equal to $J \sim 22.4, H \sim 21.3$, and $K_{s} \sim 20.0$ (CristóbalHornillos et al. 2009, 50\% of recovery efficiency depth and point-like sources). The observations in the optical range were carried out with the wide-field camera LAICA ${ }^{4}$ (four CCDs of $4096 \times 4096$ pixels and pixel scale $0.225^{\prime \prime}$ pixel $^{-1}$ ), whereas in the NIR regime they were performed with the Omega- $2000^{5}$ (one CCD of $2048 \times 2048$ pixels and plate scale $0.45^{\prime \prime}$ pixel $^{-1}$ ), both at the $3.5 \mathrm{~m}$ telescope in the Calar Alto Observatory ${ }^{6}$ (CAHA).

We adopted the ALHAMBRA Gold catalogue ${ }^{7}$ (Molino et al. 2014) as the reference photometric catalogue. This catalogue provides accurate photometry (non-fixed apertures) for developing stellar population studies of galaxies (Díaz-García et al. 2015) for $\sim 95000$ galaxies. This catalogue also contains precise photo- $z$ predictions $\left(\sigma_{z} \sim 0.012\right)$ obtained from the Bayesian Photometric Redshift (BPZ2.0; Benítez 2000; Molino et al. 2014). Our galaxy set comprises all the sources in the Gold catalogue classified as galaxies (STAR/GALAXY discriminator parameter lower than Stellar_flag $\leq 0.5$ ) and with a minimum photometric weight of $70 \%$ on the detection image (PercW $\geq 0.7$ ). This constraint removes sources that are close to the image edges. This catalogue also provides the synthetic AB-magnitude $m_{\mathrm{F} 814 \mathrm{~W}}$, which is mainly employed for detection purposes. The synthetic band $m_{\mathrm{F} 814 \mathrm{~W}}$ was not used during the SED fitting process and it sets the Gold catalogue selection as $m_{\mathrm{F} 814 \mathrm{~W}} \leq 23$ (see Molino et al. 2014).

\section{Stellar population parameters in ALHAMBRA}

Throughout this work we focus on the distribution of the stellar population parameters of quiescent galaxies within the stellar mass-colour and colour-colour diagrams. To explore this topic, we employed the photometric data of each galaxy in ALHAMBRA and the code MUFFIT (MUlti-Filter FITting for stellar population diagnostics; Díaz-García et al. 2015). Briefly, MUFFIT is a SED fitting code carefully developed to set constraints on stellar population parameters of galaxies and it is specifically optimised to deal with multi-band photometric data. MUFFIT is based on an error-weighted $\chi^{2}$-test using composite models of stellar populations (mixtures of two SSPs) and it has proved to be a powerful tool with high capabilities for constraining stellar population properties of galaxies from ALHAMBRA-like surveys (see e.g. Díaz-García et al. 2015).

As our analysis is based on galaxy colours, we included the iterative process to remove bands affected by strong emission lines, meaning all the stellar population properties reported in this work were obtained from the stellar continuum even when strong nebular or AGN emission lines are present. Moreover, MUFFIT performs Monte Carlo simulations to explore errors in the stellar population properties owing to degeneracies and photon-noise uncertainties. As a result of the SED fitting analysis performed by MUFFIT, we obtained age and metallicity (both luminosity- and mass-weighted), photo $z$ (treated as another free parameter within the $1 \sigma$ confidence level reported in the Gold catalogue), stellar mass, rest-frame luminosities, and extinction for all the galaxies in ALHAMBRA.

For the analysis, we separately built two sets of composite stellar population models from two sets of SSP models to assess

\footnotetext{
4 http://www . caha.es/CAHA/Instruments/LAICA

http://www . caha.es/CAHA/Instruments/02000

6 http://www. caha.es

http://cosmo.iaa.es/content/alhambra-gold-catalog
}

potential systematics from the use of a given population synthesis model. Firstly, we selected the Bruzual \& Charlot (2003) SSP models (hereafter BC03; Padova 1994 tracks, ages from 0.06 to $14 \mathrm{Gyr}$, and metallicities $[\mathrm{M} / \mathrm{H}]=-1.65,-0.64,-0.33,0.09$, 0.55) with a Chabrier (2003) IMF. The BC03 spectral coverage, $\lambda \lambda 91 \AA-160 \mu \mathrm{m}$, allowed us to perform our analysis in an extensive redshift range. This is relevant for this SED fitting analysis because the population of galaxies in ALHAMBRA easily extends up to redshift $z \sim 1.5$. Secondly, the other set of models comprises the Vazdekis et al. (2016, EMILES $^{8}$ ) SSP models, which includes empirical stellar spectra and is an extension of the Vazdekis et al. (2003, 2010, 2012, MIUSCAT) SSP models, where the spectral coverage extends up to $\lambda \lambda 1680 \AA-5 \mu \mathrm{m}$. It is noteworthy that this set includes the optical MIUSCAT SSP predictions (Vazdekis et al. 2012; Ricciardelli et al. 2012), the NIR predictions of MIUSCATIR (Röck et al. 2015), and the UV extension from the New Generation Stellar Library (NGSL; Koleva \& Vazdekis 2012). This updated version of the models also includes two sets of theoretical isochrones: the scaledsolar isochrones of Girardi et al. (2000; hereafter Padova00) and Pietrinferni et al. (2004, BaSTI in the following), which were taken into account for the analysis. In particular, for EMILES we took 22 ages in the range $0.05-14 \mathrm{Gyr}$, and metallicities $[\mathrm{M} / \mathrm{H}]$ $=-1.31,-0.71,-0.40,0.00,0.22$ for Padova00 and $[\mathrm{M} / \mathrm{H}]=$ $-1.26,-0.96,-0.66,-0.35,0.06,0.26,0.40$ for BaSTI, both with a Kroupa (2001) Universal IMF.

Previously to the SED fitting analysis, the photometry of the ALHAMBRA galaxies was also corrected for Milky Way dust using MUFFIT, the Schlegel et al. (1998) dust maps, and the extinction law of Fitzpatrick (1999). This extinction law covers a wider spectral range than that observed in ALHAMBRA since $z \sim 2$, and it is suitable for dereddening any photospectroscopic data. In addition, Fitzpatrick (1999) provides robust estimations of uncertainties for dereddening methodologies, which were also included in the error budget during the photometric analysis. Along this work, we only use SSP models with ages that are cosmologically relevant to build the sample of composite models of stellar populations; in other words, they cannot be much older than the age of the Universe at any redshift assuming a $\Lambda C D M$ cosmology. However, this constraint on age did not alter our results significantly. For the whole set of SSP models, extinctions were added as a foreground $\operatorname{screen}^{9}$ with values in the range $A_{V}=0.0-3.1$ (assuming a constant $R_{V}=3.1$ and the same dust attenuation in each of the composite model components), also following the extinction law of Fitzpatrick (1999) for consistency.

As there are discrepancies among the CCDs of the LAICA camera, each galaxy was analysed according to the precise photometric system or CCD in which it was imaged. For the Omega2000 camera, this process is not necessary because it only contains a unique CCD. Throughout this work, the massweighted age and metallicity $\left(\mathrm{Age}_{\mathrm{M}}\right.$ and $[\mathrm{M} / \mathrm{H}]_{\mathrm{M}}$, respectively) were preferred to the luminosity values. The mass-weighted parameters are more representative of the total stellar content of the galaxy and they are not linked to a definition of luminosity weight, although luminosity-weighted parameters were also estimated. A young population may dominate the luminosity of a galaxy, and consequently its luminosity-weighted age, even when its contribution in mass is very low (Trager et al. 2000; Conroy 2013; Vazdekis et al. 2016).

\footnotetext{
8 http://miles.iac.es

9 Throughout this work there is no distinction between extinction and attenuation law.
} 
Table 1 illustrates part of the stellar population parameters and uncertainties derived by MUFFIT using BC03 for a subset of quiescent galaxies (see Sect. 4 below) used throughout this research. The complete catalogue ${ }^{10}$ with additional data is available at the CDS.

\section{Definition of the quiescent sample}

The definition of a reliable sample of quiescent galaxies with low levels of contamination is a sensitive and tricky process because both DSF galaxies and cool stars in ground-based surveys present colours similar to those of the quiescent galaxy population. The contamination of these sources can represent a substantial part of the sample at certain redshift and mass ranges and their effects should be removed or minimised (see Sects. 4.1-4.4). Even in multi-filter photometric surveys, which are not biased by selection effects other than the photometric depth, the definition of a complete sample in stellar mass (Sect. 4.5) with accurate enough photo- $z$ predictions (Sect. 4.6) is key to reliably driving this study because the stellar mass is tightly related to the star formation history of each galaxy (e.g. Cowie et al. 1996; Trager et al. 2000; Tremonti et al. 2004; Gallazzi et al. 2005, 2006; Jimenez et al. 2007; Panter et al. 2008; González Delgado et al. 2014a,b).

\subsection{Dust-corrected UVJ diagram}

In this section we present how to diminish the contamination of DSF galaxies in our sample of quiescent galaxies using a $U V J$ diagram. Otherwise, the sample would contain a subset of younger and obscured galaxies that differs from the largely evolved quiescent galaxies. Instead of using the rest-frame $U-V$ and $V-J$ colours to build the $U V J$-diagram, we took the bands F365W, F551W, and $J$ from ALHAMBRA to compute the restframe colours $m_{\mathrm{F} 365}-m_{\mathrm{F} 551}$ and $m_{\mathrm{F} 551}-J$. We note that the effective wavelengths of these ALHAMBRA bands are the closest ones to $U, V$, and $J$. To make a reliable sample selection, we took advantage of the stellar population results provided by MUFFIT. We used both the $k$-corrected magnitudes, along with the extinction values provided by MUFFIT to build a new and improved version of the $U V J$ diagram free of dust effects, which allowed us to clean the quiescent sample of obscured star-forming galaxies. In brief, the $k$-corrected magnitudes are computed from the SED fitting analysis by convolving the ALHAMBRA photometric system with the same combination of rest-frame spectroscopic SSP models (in a general case age, metallicity, IMF, overabundances, stellar mass, and the weight of each SSP component in the mixture) that reproduces the SED of each galaxy. The uncertainties in the $k$-corrected magnitudes are also included in the analysis and they were obtained using the results from the Monte Carlo approach (for further details, see Díaz-García et al. 2015). From these mixtures, it is also straightforward to rebuild the same combination of rest-frame models with null extinction $\left(A_{V}=0.0\right)$, and therefore corrected for extinction.

In Fig. 1 we present the $U V J$ diagram corrected for reddening obtained for ALHAMBRA with the bands previously mentioned, which includes all the galaxies at $0.1 \leq z \leq 1.1$ and down to $m_{\mathrm{F} 814 \mathrm{~W}}=23$. By looking at the distribution of the rest-frame intrinsic colour $\left(m_{\mathrm{F} 365}-m_{\mathrm{F} 551}\right)_{\text {int }}$ (see insets in Fig. 1), we can easily set the limit for quiescent galaxies as $\left(m_{\mathrm{F} 365}-m_{\mathrm{F} 551}\right)_{\text {int }} \geq 1.5$, which is roughly constant with redshift

\footnotetext{
${ }^{10}$ See also http://archive.cefca.es/catalogues/alhambra_
} quiescent_galaxies-dr1 up to $z \sim 1.1$. Although this colour limit is not strictly located in the minimum between the red and blue peaks (corresponding to the quiescent and star-forming populations, respectively), its value was defined to agree with the limit established in Moresco et al. (2013, see Eq. (1) below) and to be slightly higher to avoid the now poorly populated green valley, whose limits are difficult to define due to the small number of sources. After the definition of a quiescent sample complete in stellar mass (Sect. 4.5), the sample remains almost unaltered. For comparison reasons, we present the same $U V J$ diagram without the extinction correction in Fig. 1. The range of colours defined by Moresco et al. (2013) to select quiescent galaxies (see Eq. (1)) is also shown in this diagram, which is less contaminated by obscured star-forming galaxies than the range originally proposed by Williams et al. (2009). Formally,

$$
\left\{\begin{array}{l}
\left(m_{\mathrm{F} 365}-m_{\mathrm{F} 551}\right)>0.88 \times\left(m_{\mathrm{F} 551}-J\right)+0.69, \text { for } z \leq 0.5 \\
\left(m_{\mathrm{F} 365}-m_{\mathrm{F} 551}\right)>0.88 \times\left(m_{\mathrm{F} 551}-J\right)+0.66, \text { for } z>0.5
\end{array},\right.
$$

where $m_{\mathrm{F} 365}-m_{\mathrm{F} 551}>1.6\left(m_{\mathrm{F} 365}-m_{\mathrm{F} 551}>1.5\right)$ at $z \leq 0.5(z>$ $0.5)$ and $m_{\mathrm{F} 551}-J<1.6$, all quantities at rest frame and in ABmagnitudes.

Independently of the SSP model set used, BC03 and EMILES, the extinction correction applied on the $U V J$ colours yielded striking results. Firstly, and as expected, removing dust effects makes the colour bimodality of galaxies clearer in the $U V J$ diagram (see Fig. 1). This occurs because a great part of the galaxies in the green valley (the bridge between the red and blue galaxies) are obscured star-forming galaxies. If we define the green valley as those galaxies whose colours are close to the limiting values expressed by Eq. (1), more precisely $\pm 0.1 \mathrm{mag}$, we find that a $\sim 30 \%$ fraction of the galaxies are actually obscured star-forming galaxies. For other definitions of the green valley, this amount can be even higher. Their intrinsic colour $\left(m_{\mathrm{F} 365}-m_{\mathrm{F} 551}\right)_{\text {int }}$ reveals that these galaxies really lie within the star-forming population, although they also present a large dust content that reddens their observed colours (Brammer et al. 2009; Whitaker et al. 2010, obtained a similar result for $U-V)$. This means that the green valley is largely depopulated after accounting for extinction (Fig. 1; see also Bell et al. 2005; Cowie \& Barger 2008; Brammer et al. 2009; Cardamone et al. 2010).

Secondly, and for a sample complete down to $m_{\mathrm{F} 814 \mathrm{~W}}=$ 23 , the histogram of the $\left(m_{\mathrm{F} 365}-m_{\mathrm{F} 551}\right)_{\text {int }}$ colour exhibits a local minimum at $\sim 1.45$, which can be imposed as the bluest colour limit to fairly select quiescent galaxies in ALHAMBRA. This limit to separate quiescent from star-forming galaxies also remains roughly constant since $z \leq 1.1$, and it does not present any remarkable evolution. In spite of the presence of a bridge between the bulk of intrinsic red and blue galaxies at $\left(m_{\mathrm{F} 365}-m_{\mathrm{F} 551}\right)_{\text {int }} \sim 1.45$, this colour range is not populated by many galaxies.

Thirdly, those galaxies labelled as quiescent by Eq. (1) and that belong to the star-forming sample after the dust correction (intrinsic $m_{\mathrm{F} 365}-m_{\mathrm{F} 551}<1.5$, see Fig. 1) are typically concentrated close to the edges of Eq. (1), supporting the reliability of the extinction values provided by MUFFIT. Otherwise, the distribution of DSF galaxies would uniformly populate the red part of the $U V J$ diagram as a consequence of degeneracies, where the effects of age, metallicity, and extinction on the stellar continuum would not be properly differentiated.

Finally, DSF galaxies comprise $\sim 20 \%$ of the quiescent sample defined through Eq. (1) (see Fig. 1). Our results clearly establish that more massive quiescent galaxies are less biased 
L. A. Díaz-García et al.: Stellar populations of galaxies within diagrams involving colours

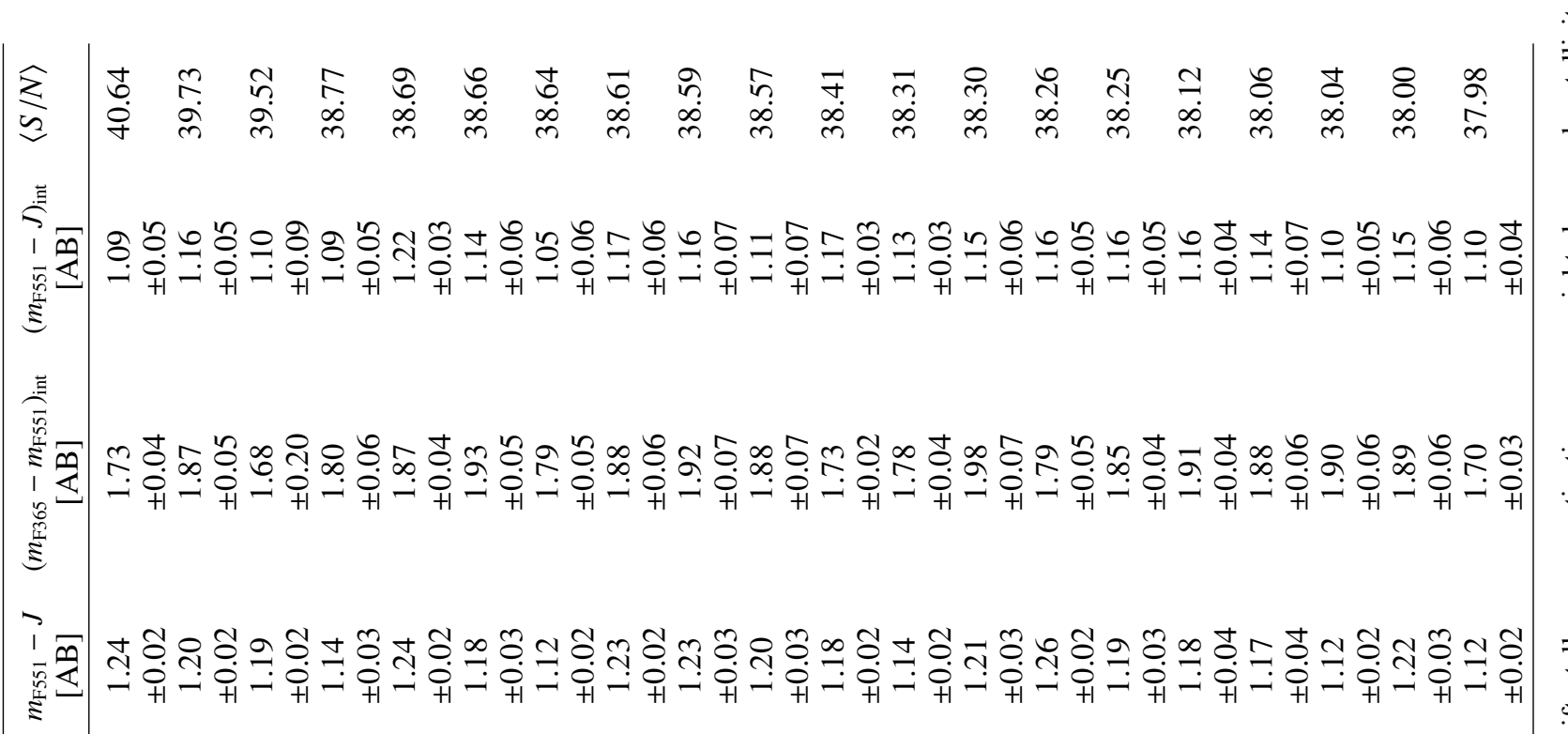

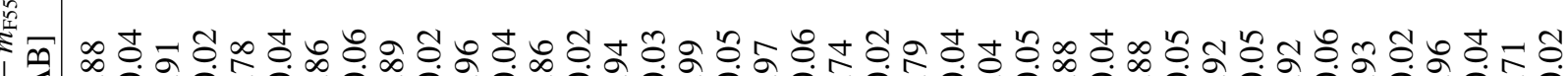

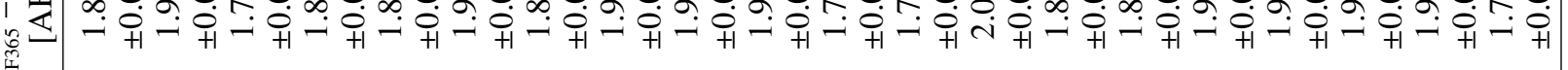

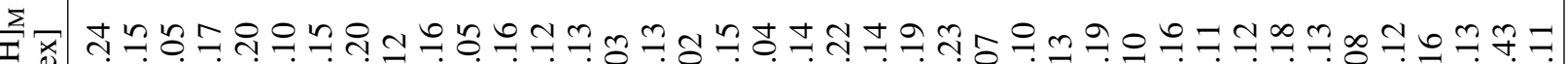

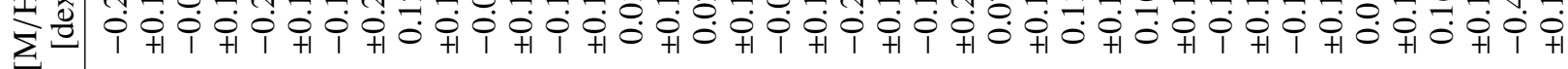

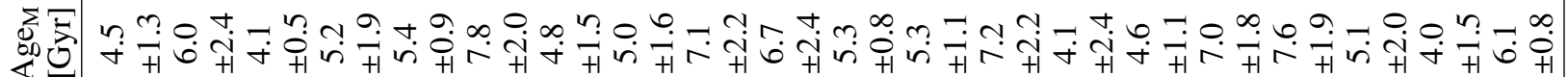

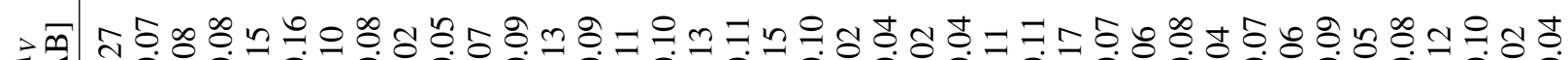

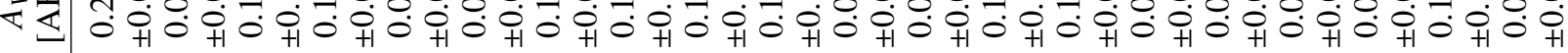

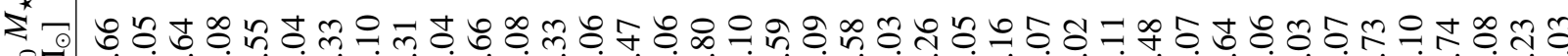

on 2000

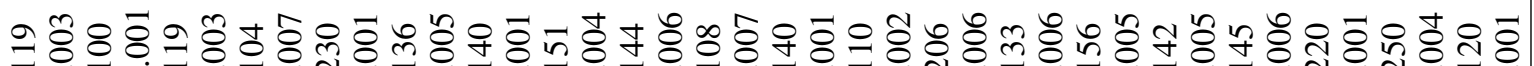
0

ชา ㅈำกำㅇำ

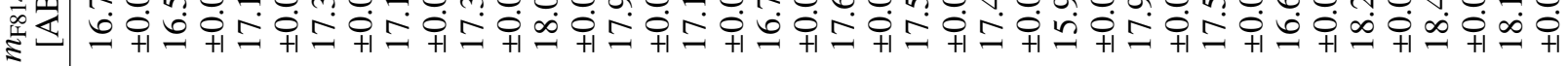



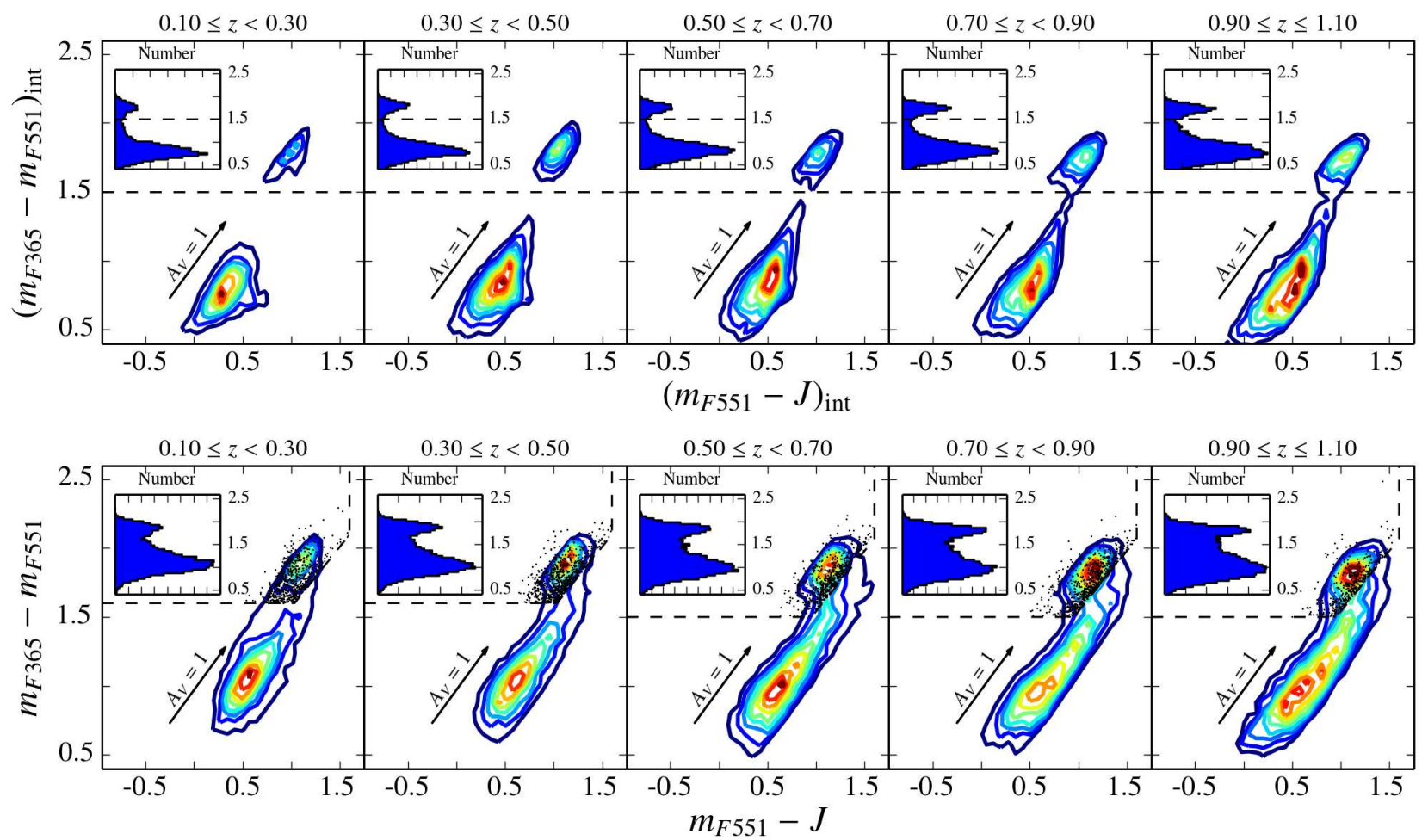

Fig. 1. Density surface and distribution of rest-frame colours for galaxies from the ALHAMBRA survey obtained using BC03 SSP models. Top: rest-frame intrinsic colours $\left(m_{\mathrm{F} 551}-J\right)_{\text {int }}(X$-axis $)$ and $\left(m_{\mathrm{F} 365}-m_{\mathrm{F} 551}\right)_{\text {int }}(Y$-axis $)$ after correcting for extinction at different redshifts. Bottom: restframe colours without removing dust effects. Redder (bluer) density-curve colours are related to higher (lower) densities. Inner panels, histograms of the intrinsic (top) and observed (bottom) rest-frame colour $m_{\mathrm{F} 365}-m_{\mathrm{F} 551}$. Dashed lines in the top panels illustrate our limiting value ( $m_{\mathrm{F} 365}-$ $\left.m_{\mathrm{F} 551}\right)_{\text {int }}=1.5$ for quiescent galaxies, and in the bottom panels the quiescent $U V J$ sample defined by Moresco et al. (2013, Eq. (1)). Black dots are galaxies labelled as quiescent by the $U V J$ criteria of Moresco et al. (2013) that lie in the star-forming region after removing extinction effects. We illustrate the colour variations owing to a reddening of $A_{V}=1$ (black arrow), assuming the extinction law of Fitzpatrick (1999).

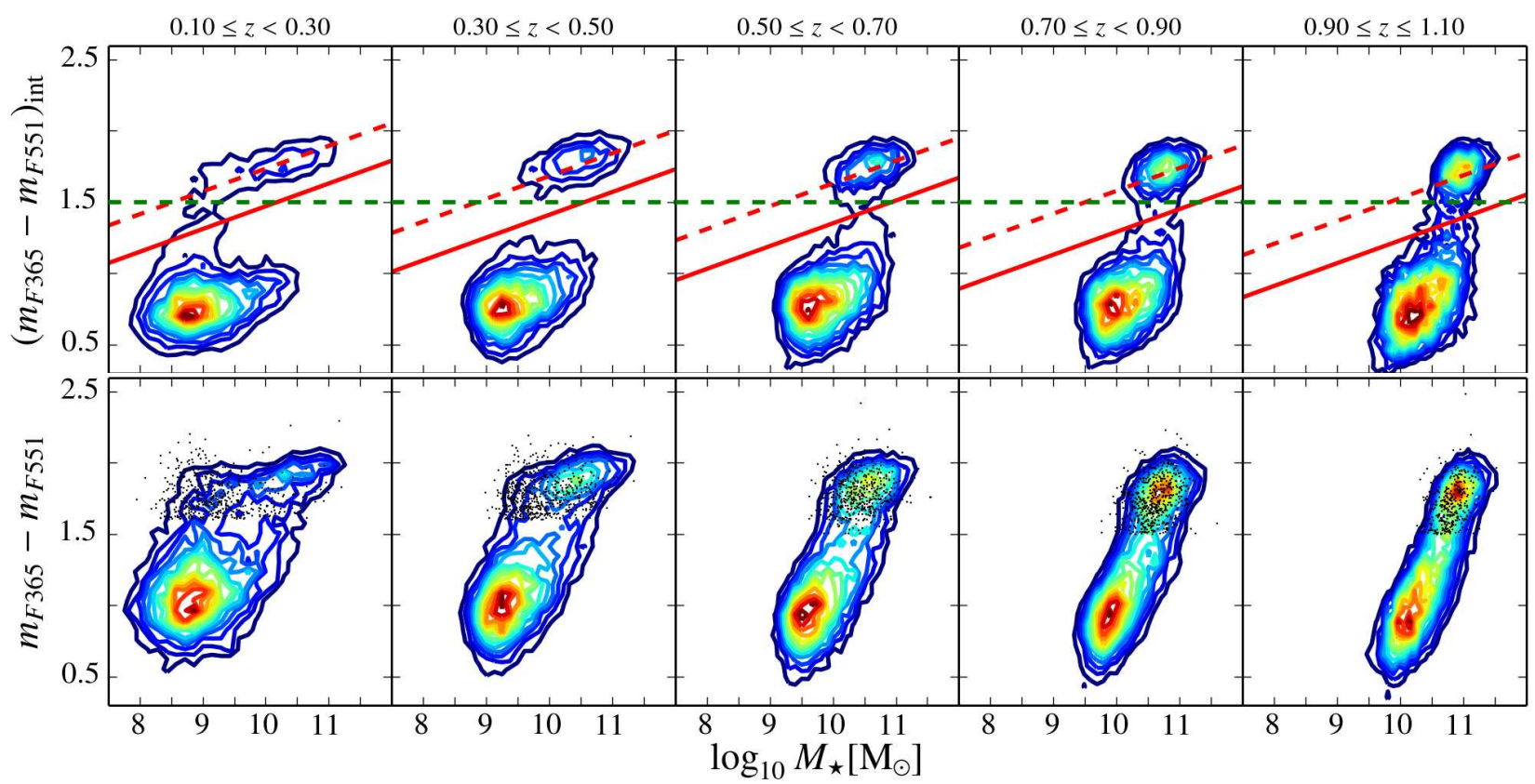

Fig. 2. Density surface and distribution of stellar mass vs. rest-frame colour $\left(m_{\mathrm{F} 365}-m_{\mathrm{F} 551}\right)$ for galaxies from the ALHAMBRA survey using BC03 SSP models. Top: rest-frame intrinsic colour $\left(m_{\mathrm{F} 365}-m_{\mathrm{F} 551}\right)_{\text {int }}(Y$-axis $)$ after correcting for extinction at different redshift. Bottom: rest-frame colours without removing dust effects. Redder (bluer) density-curve colours are related to high (low) densities. Dashed green lines in the top panels illustrate the limiting value $\left(m_{\mathrm{F} 365}-m_{\mathrm{F} 551}\right)_{\text {int }}=1.5$ used for selecting quiescent galaxies in Sect. 4.1. Dashed and solid red lines show the fit to the quiescent sequence, $\left(m_{\mathrm{F} 365}-m_{\mathrm{F} 551}\right)_{\mathrm{int}}^{\mathrm{Q}}$, and the limiting intrinsic colours, $\left(m_{\mathrm{F} 365}-m_{\mathrm{F} 551}\right)_{\mathrm{int}}^{\lim }$, values of quiescent galaxies respectively (see details in Sect. 4.2). Black dots in the bottom panels are galaxies labelled as quiescent with the $U V J$ criteria of Moresco et al. (2013) that lie in the star-forming region after removing extinction effects. 
Table 2. Percentage of red $U V J$ galaxies (defined by Eq. (1)) in ALHAMBRA with a bluer intrinsic colour than $\left(m_{\mathrm{F} 365}-m_{\mathrm{F} 551}\right)_{\text {int }}<1.5$ after a dust correction at different redshift and stellar mass bins.

\begin{tabular}{rccccc}
\hline \hline & $0.1 \leq z<0.3$ & $0.3 \leq z<0.5$ & $0.5 \leq z<0.7$ & $0.7 \leq z<0.9$ & $0.9 \leq z \leq 1.1$ \\
\hline $9.6 \leq \log _{10} M_{\star}<10.0$ & 24.8 & - & - & - & - \\
$10.0 \leq \log _{10} M_{\star}<10.4$ & 15.3 & 14.8 & - & - & - \\
$10.4 \leq \log _{10} M_{\star}<10.8$ & 5.9 & 8.1 & 19.2 & - & - \\
$10.8 \leq \log _{10} M_{\star}<11.2$ & 5.1 & 2.8 & 11.2 & 11.6 & - \\
$\log _{10} M_{\star} \geq 11.2$ & 7.3 & 6.0 & 5.4 & 4.0 & 6.2 \\
\hline
\end{tabular}

by DSF galaxies than the less massive ones (see Fig. 2 as well). More precisely, the most massive part of the sample is weakly contaminated by DSF galaxies when they are defined by Eq. (1) $\left(\log _{10} M_{\star} \geq 11,4-10 \%\right.$ from $z \sim 0.1$ to $\left.z \sim 1.1\right)$, while the lowmass ones may be significantly biased by them (e.g. $\sim 40 \%$ for $9.2 \leq \log _{10} M_{\star} \leq 9.6$ at $0.1 \leq z \leq 0.3$ ). In Table 2 , we illustrate the number of galaxies labelled as DSF galaxies at different redshift and stellar mass bins, meaning the level of contamination that we would expect when we build samples of quiescent galaxies via red $U V J$ colour-colour diagrams such as Eq. (1) (see also Moresco et al. 2013).

In addition to the colour cut $\left(m_{\mathrm{F} 365}-m_{\mathrm{F} 551}\right)_{\text {int }} \geq 1.5$, we restrict this study to the redshift interval $0.1 \leq z \leq 1.1$ because the stellar mass completeness constraint largely reduces the number of quiescent galaxies further than $z>1.1$ and because at $z<0.1$ the number of quiescent galaxies in ALHAMBRA is also very low, especially for the most massive ones, which are substantially less frequent. The total number of quiescent galaxies at this point is $\sim 15500$, where discrepancies in number owing to the use of BC03 and EMILES are lower than $2 \%$.

\subsection{Stellar mass-colour diagram corrected for extinction}

One of the main goals of $U V J$-like diagrams is to reduce the number of DSF galaxies in quiescent galaxy samples (see e.g. Moresco et al. 2013). We show above that removing dust effects makes the colour bimodality of galaxies clearer in the $U V J$ diagram (Fig. 1). However, we did not know whether the selection in this diagram is creating a stellar-mass bias in the quiescent sample. For this reason, we explored the rest-frame stellar masscolour diagram corrected for extinction (MCDE). This diagram was conceived to both unmask the DSF galaxies and include the stellar mass of galaxies (physical parameter) avoiding any bias in the sample selection. The MCDE diagram is actually a step forward or improvement with respect to classical CMD diagrams (e.g. Bell et al. 2004; Baldry et al. 2004; Brown et al. 2007) or the stellar mass-colour diagram (e.g. Peng et al. 2010; Schawinski et al. 2014, see Fig. 2), which are typically contaminated by DSF galaxies.

As expected, the MCDE makes the colour bimodality of galaxies clearer (see Fig. 2), where the dust-corrected colour $\left(m_{\mathrm{F} 365}-m_{\mathrm{F} 551}\right)_{\text {int }}$ is the key to disentangling quiescent galaxies from the red sources that are actually DSF galaxies (see Fig. 2). Otherwise, disentangling DSF galaxies via colour-colour diagrams or CMD is not completely reliable. Again, the presence of galaxies in the green valley is much rarer, pointing out the large presence of DSF galaxies in the green valley colour range when a dust correction is not performed. Figure 2 shows that the colour cut $\left(m_{\mathrm{F} 365}-m_{\mathrm{F} 551}\right)_{\text {int }} \geq 1.5$ defined in Sect. 4.1 properly selects quiescent galaxies at first order up to $z \sim 1$ and $m_{\mathrm{F} 814} \leq 23$, although only for this work. For a general case, we deal with this concern in more detail in Sect. 7.2. However, to better define the colour limit of the quiescent galaxy population (see Fig. 2) we follow a process of three steps:

(i) From the distribution of ALHAMBRA galaxies on the MCDE, we defined a set of straight lines to separate the two populations by eye. As in Sect. 4.1, we focus on the redshift range at $0.1 \leq z \leq 1.1$

(ii) We stated the representative intrinsic colour $\left(m_{\mathrm{F} 365}-\right.$ $\left.m_{\mathrm{F} 551}\right)_{\text {int }}$ of quiescent galaxies as a function of stellar mass and redshift, denoted as $\left(m_{\mathrm{F} 365}-m_{\mathrm{F} 551}\right)_{\mathrm{int}}^{\mathrm{Q}}$. To do this, we performed a least-squares fitting with all the quiescent galaxies above the subsample defined by eye in the previous step, that is, the red galaxies in the MCDE. For the fit we assumed a linear relation between the intrinsic colour $\left(m_{\mathrm{F} 365}-m_{\mathrm{F} 551}\right)_{\text {int }}$ and stellar mass;

(iii) We set colour limits for quiescent galaxies, $\left(m_{\mathrm{F} 365}-\right.$ $\left.m_{\mathrm{F} 551}\right)_{\text {int }}$ as a function of redshift. To set this limit, we assumed that it exhibits the same dependence on stellar mass as $\left(m_{\mathrm{F} 365}-m_{\mathrm{F} 551}\right)_{\text {int }}^{\mathrm{Q}}$. Nevertheless, the ordinate depends on the intrinsic colour spread, $\left(m_{\mathrm{F} 365}-m_{\mathrm{F} 551}\right)_{\mathrm{int}}^{\mathrm{rot}}$, of quiescent galaxies,

$$
\left(m_{\mathrm{F} 365}-m_{\mathrm{F} 551}\right)_{\mathrm{int}}^{\mathrm{rot}}=\left(m_{\mathrm{F} 365}-m_{\mathrm{F} 551}\right)_{\mathrm{int}}-\left(m_{\mathrm{F} 365}-m_{\mathrm{F} 551}\right)_{\mathrm{int}}^{\mathrm{Q}} \text {, }
$$

which is properly described by a log-normal distribution (see Appendix A). As this distribution is affected by uncertainties, which also vary with redshift, we adopted the maximum likelihood method (MLE) developed by LópezSanjuan et al. (2014, further details in Appendix A) to remove uncertainty effects and reveal the intrinsic distribution of values. We define the maximum $\left(m_{\mathrm{F} 365}-m_{\mathrm{F} 551}\right)_{\text {int }}$ value of quiescent galaxies as the $3 \sigma$ limit of the log-normal distribution revealed from the MLE.

As a result, we obtain that the representative intrinsic colours $\left(m_{\mathrm{F} 365}-m_{\mathrm{F} 551}\right)_{\mathrm{int}}^{\mathrm{Q}}$ and the limiting values $\left(m_{\mathrm{F} 365}-m_{\mathrm{F} 551}\right)_{\mathrm{int}}^{\lim }$ of quiescent galaxies are both properly expressed by an equation of the form

$\left(m_{\mathrm{F} 365}-m_{\mathrm{F} 551}\right)_{\mathrm{int}}^{\mathrm{Q}, \lim }=a \cdot\left(\log _{10} M_{\star} / M_{\odot}-10\right)+b \cdot(z-0.1)+c$,

where $a, b$, and $c$ depend on the SSP model set used. In Table 3 , we show the values $a, b$, and $c$ obtained for $\left(m_{\mathrm{F} 365}-\right.$ $\left.m_{\mathrm{F} 551}\right)_{\mathrm{int}}^{\mathrm{Q}}$ and $\left(m_{\mathrm{F} 365}-m_{\mathrm{F} 551}\right)_{\mathrm{int}}^{\mathrm{lim}}$ using BC03, EMILES+BaSTI, and EMILES+Padova00 SSP models.

From this analysis and the distribution of colours of quiescent galaxies in the MCDE, we find that (i) the slope of the quiescent sequence in this diagram is compatible with a constant value of $\sim 0.16$ since $z \sim 1$; (ii) the colour spread of values $\left(m_{\mathrm{F} 365}-m_{\mathrm{F} 551}\right)_{\text {int }}$ has not changed significantly since $z \sim 1$, which exhibits a value in the range $\sim 0.26-0.28$ (AB magnitudes, $3 \sigma$ limit; see Table 3); (iii) the selection of green valley galaxies is subject to a higher contamination of DSF galaxies when making use of the CMD; and (iv) this contamination of DSF galaxies 
Table 3. Parameters $a, b$, and $c$ that best fit the representative intrinsic colours $\left(m_{\mathrm{F} 365}-m_{\mathrm{F} 551}\right)_{\mathrm{int}}^{\mathrm{Q}}($ top panel $)$ and the limiting values $\left(m_{\mathrm{F} 365}-\right.$ $\left.m_{\mathrm{F} 551}\right)_{\text {int }}^{\lim }$ (bottom panel) of quiescent galaxies using Eq. (3) and the SSP models of BC03 and EMILES (BaSTI and Padova00 isochrones).

\begin{tabular}{lccc}
\hline \hline$\left(m_{\mathrm{F} 365}-m_{\mathrm{F} 551}\right)_{\mathrm{int}}^{\mathrm{Q}}$ & $a$ & $b$ & $c$ \\
\hline BC03 & $0.16 \pm 0.01$ & $-0.26 \pm 0.12$ & $1.76 \pm 0.15$ \\
BaSTI & $0.15 \pm 0.01$ & $-0.24 \pm 0.12$ & $1.73 \pm 0.15$ \\
Padova00 & $0.15 \pm 0.01$ & $-0.19 \pm 0.11$ & $1.75 \pm 0.15$ \\
\hline$\left(m_{\mathrm{F} 365}-m_{\mathrm{F} 551}\right)_{\mathrm{int}}^{\mathrm{lim}}$ & $a$ & $b$ & $c$ \\
\hline BC03 & $0.16 \pm 0.01$ & $-0.30 \pm 0.26$ & $1.50 \pm 0.18$ \\
BaSTI & $0.15 \pm 0.01$ & $-0.24 \pm 0.17$ & $1.46 \pm 0.15$ \\
Padova00 & $0.15 \pm 0.01$ & $-0.13 \pm 0.06$ & $1.47 \pm 0.13$ \\
\hline
\end{tabular}

depends on the SSP model set used and amounts to 50-75\%. For further results concerning green valley galaxies, see Sect. 7.1.

\subsection{Visual inspection}

To increase the purity of the sample, we also carried out an individual inspection of the $\sim 15000$ galaxies with intrinsic red colours obtained in Sects. 4.1 and 4.2 at $0.1 \leq z \leq 1.1$. We removed from the sample spurious detections and those galaxies that were compromised by very nearby sources and/or were imaged in bad CCD regions. To develop this process, we simultaneously checked one-by-one all the galaxy stamps, the adequacy of the photometric aperture (mainly the efficiency on the detection-deblending of sources and surroundings), and that the photo-spectrum did not present strong irregularities such as time-variable sources or sources close to stellar spikes, which cannot be reproduced by stellar population models. In the end we removed $\sim 5 \%$ of sources from the quiescent sample after the visual inspection.

\subsection{Removal of cool stars}

The ALHAMBRA Gold catalogue provides a statistical star/galaxy classification to understand whether each source in the catalogue is a galaxy or a star (Stellar_flag, see details in Molino et al. 2014). This parameter was originally used to define our sample of galaxies (see Sect. 2) and it simultaneously accounts for the morphology, apparent magnitude, and two colours to provide a statistical approach. Unfortunately, this parameter is less effective at decreasing the signal-to-noiseratio, and consequently this classification is uncertain for sources fainter than $m_{\mathrm{F} 814 \mathrm{~W}}=22.5$, and a value Stellar_flag $=0.5$ is assigned. Although we do not expect a large degree of contamination from stars at $22.5 \leq m_{\mathrm{F} 814 \mathrm{~W}}<23$ for the full sample, the ALHAMBRA fields may contain stars from the Milky Way halo, which are mainly composed of cool red stars. Our quiescent sample, composed of red sources, may therefore be partly contaminated by stars that were treated as galaxies.

This problem was faced through a new MUFFIT module devoted to analysing stars. This performs a SED fitting process that is similar to the version for galaxies, but using the Coelho et al. (2005) star models instead. This methodology reduced the contamination of cool stars in ALHAMBRA from $24 \%$ to $4 \%$ after comparing our faint star detection predictions with the star/galaxy classification provided by the Cosmological Evolution Survey (COSMOS, classified as point-like sources thanks to its tiny PSF; Leauthaud et al. 2007) in a shared subsample

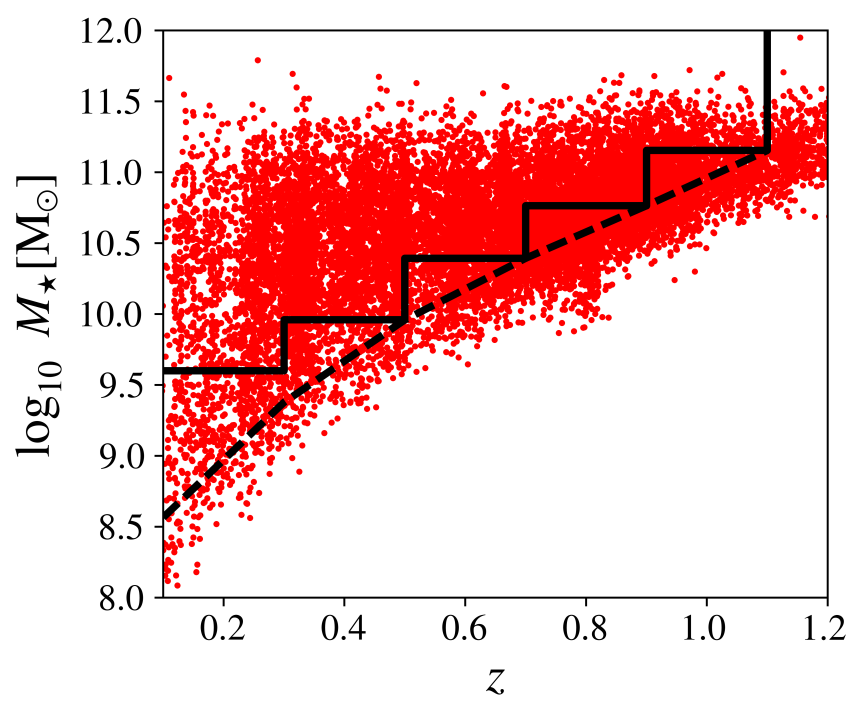

Fig. 3. Redshifts ( $X$-axis) and stellar masses ( $Y$-axis) of ALHAMBRA quiescent galaxies. The dashed line illustrates the 95\% stellar mass completeness level of quiescent galaxies from the ALHAMBRA Gold catalogue (complete down to $m_{\mathrm{F} 814 \mathrm{~W}}=23$ ). The solid line illustrates the limits assumed in this work to define our sample of galaxies complete in stellar mass at different redshift bins (see Sect. 4).

of red sources at $22.5 \leq m_{\mathrm{F} 814 \mathrm{~W}}<23$. It is worth mentioning that owing to the spectral coverage of the Coelho et al. (2005) stellar models ( $300 \mathrm{~nm}-1.8 \mu \mathrm{m})$, the flux from the ALHAMBRA $K_{s}$ band is not used for the stellar SED fitting analysis. A more extended explanation of the whole process, as well as the comparison with COSMOS to check the reliability of the methodology, is detailed in Appendix B. In the end, we removed 439 star candidates from the sample at $22.5 \leq m_{\mathrm{F} 814 \mathrm{~W}}<23$.

\subsection{Stellar mass completeness}

We modelled the stellar mass completeness, $C$, through a FermiDirac distribution (see Appendix C) that is parametrised by two redshift-dependent parameters, $M_{F}$ and $\Delta_{F}$. The parameter $M_{F}$ reflects the stellar mass value (in dex units) for which the completeness is equal to $50 \%(C=0.5)$, while $\Delta_{F}$ is related to the rate of decrease in the number of galaxies. We found that this kind of distribution properly reproduces the decay on the less massive galaxies of our flux-limited sample $\left(m_{\mathrm{F} 814 \mathrm{~W}} \leq 23\right)$ at any redshift. The process used to derive these parameters takes advantage of stellar mass functions from deeper surveys (for further details, see Appendix C), in particular from the COSMOS survey, which specifically provides them for quiescent galaxies (Ilbert et al. 2010, computed using BC03 SSP models) and partly overlaps with ALHAMBRA. In this process, we assumed that any discrepancy between the low-mass end of the ALHAMBRA stellar mass function and the COSMOS value was caused by the mass incompleteness. These discrepancies allowed us to determine both $M_{F}$ and $\Delta_{F}$, which are directly related to the stellar mass limit, $\log _{10} M_{C}$, at redshift $z$ and completeness level by

$\log _{10} M_{C}(z)=M_{F}(z)+\Delta_{F}(z) \ln \left[\frac{C}{1-C}\right]$.

For this work, we required a conservative stellar mass completeness of at least $C=0.95$ at any redshift bin (see Fig. 3 and values in Table 4). From Table 4 we derive that ALHAMBRA is complete up to $\log _{10} M_{\star} \geq 9.4$ dex at $z=0.3$. However, to develop this work, we increased this mass limit to 
Table 4. Parameters $M_{F}$ and $\Delta_{F}$ at different redshifts, and the stellar mass limit at the completeness levels $C=0.7,0.8,0.9$, and 0.95 for the quiescent sample and BC03 SSP models (details in text).

\begin{tabular}{lccccc}
\hline \hline Redshift & 0.3 & 0.5 & 0.7 & 0.9 & 1.1 \\
\hline$M_{F}$ & 8.99 & 9.64 & 10.08 & 10.44 & 10.84 \\
$\Delta_{F}$ & 0.132 & 0.108 & 0.105 & 0.110 & 0.108 \\
$\log _{10} M_{C=0.7}$ & 9.10 & 9.73 & 10.17 & 10.53 & 10.93 \\
$\log _{10} M_{C=0.8}$ & 9.17 & 9.79 & 10.23 & 10.59 & 10.99 \\
$\log _{10} M_{C=0.9}$ & 9.28 & 9.88 & 10.31 & 10.68 & 11.07 \\
$\log _{10} M_{C=0.95}$ & 9.38 & 9.96 & 10.39 & 10.76 & 11.15 \\
\hline
\end{tabular}

Notes. Parameters for EMILES SSP models are obtained adding 0.15 and 0.11 dex to $M_{F}$ for BaSTI and Padova00 isochrones, respectively. For EMILES, $\Delta_{F}$ is equal to the $\mathrm{BC} 03$ values.

$\log _{10} M_{\star} \geq 9.6$ dex, with the only aim of having a set of equalsized stellar mass bins of $\sim 0.4$ dex. In Appendix $C$, there is a more detailed and complete explanation of the full process used to determine the ALHAMBRA completeness. When stellar masses are obtained using the MUFFIT and EMILES SSP models, their values differ with respect to those obtained using BC03 SSP models. EMILES stellar masses are systematically higher by about 0.15 and 0.11 dex for BaSTI and Padova00 isochrones, respectively. To determine the stellar mass completeness of EMILES predictions, we add 0.15 (EMILES+BaSTI) and 0.11 dex (EMILES+Padova00) to the $M_{F}$ values provided in Table 4, whereas $\Delta_{F}$ remains unaltered. We checked that this shift analytically reproduces the observed stellar masses properly.

Finally, the total number of galaxies for this study, including the stellar mass completeness constraint, comprises a total of $\sim 8500$ quiescent galaxies for BC03 SSP models (all the galaxies in our sample are illustrated in Fig. 3). The number of quiescent galaxies per stellar mass and redshift bin is detailed in Table 5. For EMILES SSP models, the final number of quiescent galaxies for this work is $\sim 8100$.

\subsection{Photo-z accuracy of ALHAMBRA quiescent galaxies}

An accurate photo- $z$ determination is essential to properly drive a stellar population study, otherwise any stellar population prediction may be erroneous. As we mention in Sect. 3, we initially used the photo- $z$ constraints provided in the ALHAMBRA Gold catalogue (computed using BPZ2.0) as input for the SED fitting analysis. Therefore, we ran MUFFIT in the photo$z$ intervals provided in this catalogue, treating the redshift as another free parameter to determine during our stellar population analysis.

In this section we describe how we determined the photo- $z$ precision obtained for the sample of quiescent galaxies studied in this work. To this end, we took advantage of the same subsample of spectroscopic galaxies used by Molino et al. (2014; priv. comm.), which contains publicly available spectroscopic redshifts from surveys that overlap with ALHAMBRA (zCOSMOS; Lilly et al. 2009; AEGIS $^{11}$, Davis et al. 2007; and GOODS-N ${ }^{12}$, Cooper et al. 2011). This spectroscopic subsample comprises galaxies at $0.0<z<1.5$ and magnitudes $18<m_{\mathrm{F} 814 \mathrm{~W}}<25$ (mean values of $\langle z\rangle \sim 0.77$ and $\left\langle m_{\mathrm{F} 814 \mathrm{~W}}\right\rangle \sim 22.3$ ). Because there is no standard method for setting numerical values of the photo$z$ accuracy, we turned to various statistical estimators already

\footnotetext{
11 All-wavelength Extended Groth strip International Survey.
}

12 The Great Observatories Origins Deep Survey-North. used in the literature. The most extended one is probably the normalised median absolute deviation $\left(\sigma_{\mathrm{NMAD}}\right.$, Brammer et al. 2008) since this estimator is less affected by catastrophic errors or outliers. Formally,

$\sigma_{\mathrm{NMAD}}=1.48 \times \operatorname{median}\left(\frac{|\Delta z-\operatorname{median}(\Delta z)|}{1+z_{\mathrm{spec}}}\right)$,

where $\Delta z=z_{\text {phot }}-z_{\text {spec }}$. Moreover, we propose here another photo- $z$ accuracy estimator: the root mean square (rms) of the Gaussian distribution built from the values $\Delta z /\left(1+z_{\text {spec }}\right)$, which in the following we denote as $\sigma_{\text {photo-z }}$. The number of catastrophic outliers is also an important factor to take into account, so we propose two definitions for it:

$\eta_{1}=\frac{|\Delta z|}{1+z_{\text {spec }}}>0.2$, and
$\eta_{2}=\frac{|\Delta z|}{1+z_{\text {spec }}}>5 \times \sigma_{\mathrm{NMAD}}$.

Amongst the $\sim 8500$ quiescent galaxies in our sample complete in stellar mass (see Sect. 4.5 above), there are 576 quiescent galaxies with spectroscopic redshifts. Figure 4 illustrates the one-to-one comparison of these 576 quiescent galaxies, showing the excellent agreement between our photometric predictions and the spectroscopic predictions. For this subsample of 576 galaxies and according to Eqs. (5)-(7), we obtained $\sigma_{\mathrm{NMAD}}=$ $0.0064, \eta_{1}=0.5 \%$, and $\eta_{2}=6.6 \%$, respectively. Regarding the $\Delta z /\left(1+z_{\mathrm{spec}}\right)$ distribution, we fitted this distribution adopting a Gaussian-like function (see Fig. 4). The Gaussian fit is centred at 0.0006 , which is closely centred to zero or negligible shift, and exhibits an rms of $\sigma_{\text {photo-z }}=0.0053$.

If we compare the photo- $z$ values provided by BPZ2.0 in the ALHAMBRA Gold catalogue with the spectroscopic values, we obtain an accuracy of $\sigma_{\mathrm{NMAD}}=0.0080, \sigma_{\text {photo-z }}=0.0062, \eta_{1}=$ $0.5 \%$, and $\eta_{2}=7.1 \%$. Therefore, adopting photo- $z$ constraints from external dedicated codes during the MUFFIT SED fitting analysis may improve the photo- $z$ accuracy by $\sim 15-20 \%$. As checked by Díaz-García et al. (2015), a photo- $z$ uncertainty at this level $(0.6 \%)$ has a minimum effect on the stellar population parameters that are determined via SED fitting in ALHAMBRA: stellar mass, age, metallicity, and extinction.

\section{Star formation rates via SED fitting results}

Our SED fitting code MUFFIT uses two SSPs for the fitting, which by definition has null SFR since it involves two SSPs of non-zero age (unlike other SED fitting analyses based on $\tau$ models or in more complex SFHs, see e.g. Cid Fernandes et al. 2005; Moustakas et al. 2013). In order to obtain SFRs from the SED fitting results provided by MUFFIT, it is necessary to define a tracer or parameter that allows us to estimate them. Although the SFR of quiescent galaxies is typically low, it can also be used to complement and reinforce the reliability of the results obtained in Sects. 4.1 and 4.2. If red galaxies labelled as DSF galaxies after the dust correction (see Eq. (1)) also show SFRs proper of star-forming galaxies, we can be more confident that these galaxies were removed from the quiescent sample properly. In this section we propose a methodology for providing SFR values of galaxies based on the SED fitting results provided by MUFFIT via the $2800 \AA$ luminosity (SFR tracer, see e.g. Kennicutt 1998; Madau et al. 1998).

The UV continuum in the range $\lambda \lambda 1500-2800 \AA$ is a good tracer of SFR in galaxies with ongoing star formation, because 
Table 5. Number of quiescent galaxies per stellar mass and redshift bin.

\begin{tabular}{rrcccccc}
\hline \hline & & $0.1 \leq z<0.3$ & $0.3 \leq z<0.5$ & $0.5 \leq z<0.7$ & $0.7 \leq z<0.9$ & $0.9 \leq z \leq 1.1$ & Total \\
\hline $9.6 \leq \log _{10}$ & $M_{\star}<10.0$ & 289 & - & - & - & - & 289 \\
$10.0 \leq \log _{10}$ & $M_{\star}<10.4$ & 433 & 996 & - & - & - & 1429 \\
$10.4 \leq \log _{10}$ & $M_{\star}<10.8$ & 403 & 1035 & 1088 & - & - & 2526 \\
$10.8 \leq \log _{10}$ & $M_{\star}<11.2$ & 238 & 663 & 800 & 1480 & - & 3181 \\
$\log _{10}$ & $M_{\star} \geq 11.2$ & 51 & 140 & 157 & 357 & 417 & 1122 \\
& Total & 1414 & 2834 & 2045 & 1837 & 417 & 8547 \\
\hline
\end{tabular}

Notes. The last column gives the total number of galaxies per stellar mass bin. The last row shows the total number of quiescent galaxies per redshift bin. All the cells include redshift and stellar mass bins complete at the level $C=0.95$, otherwise there is a dash.
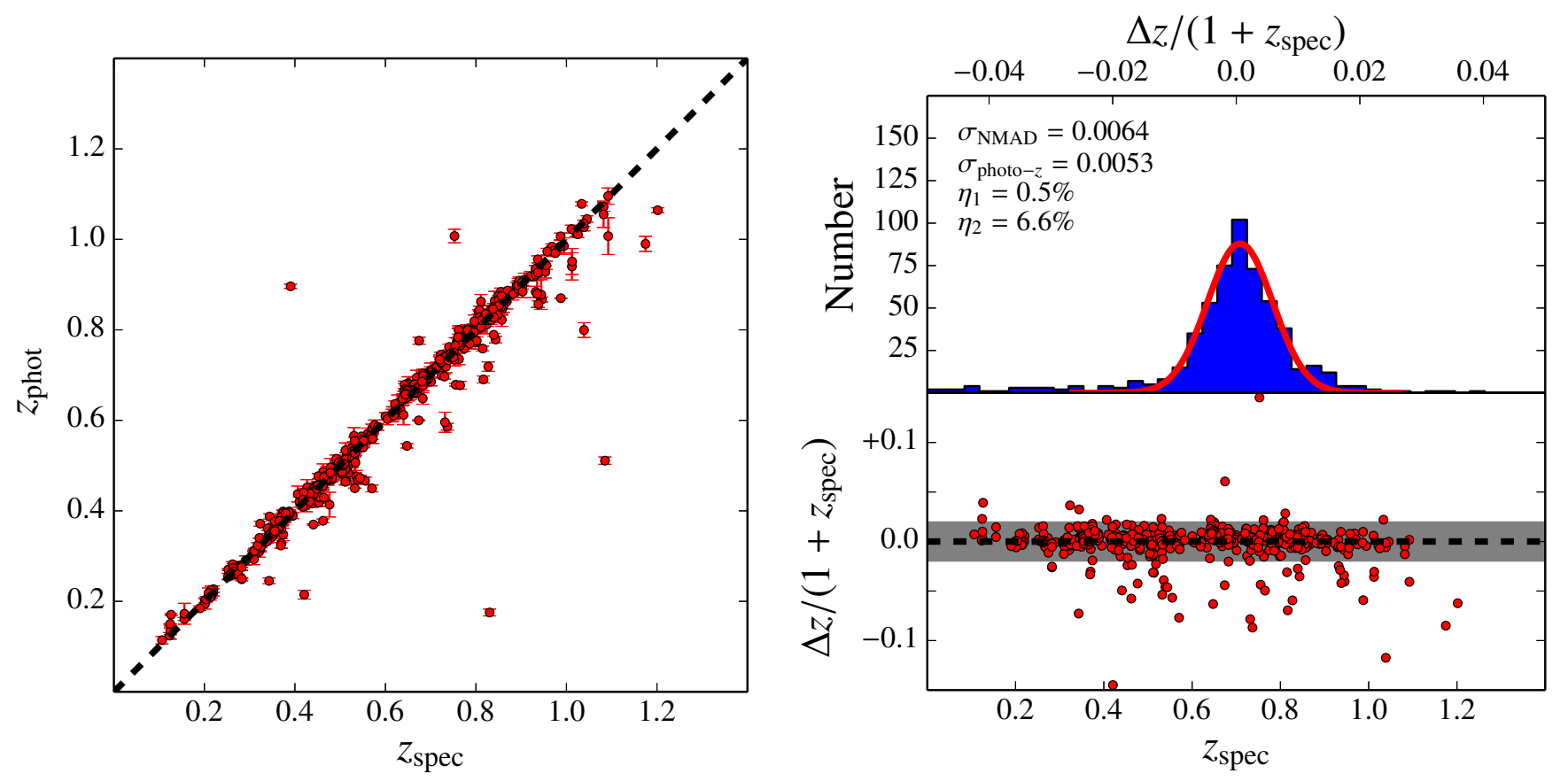

Fig. 4. Comparison between the photo- $z$ provided by MUFFIT $\left(z_{\text {phot }}\right)$ and their spectroscopic values $\left(z_{\text {spec }}\right)$ for 576 shared quiescent galaxies. Left panel: one-to-one comparison of redshifts, where dashed black line is the one-to-one relation. Top right: histogram of values $\Delta z /\left(1+z_{\text {spec }}\right)$, along with the Gaussian that best fits this distribution (red solid line) and the photo-z accuracy estimators defined in the text (Eqs. (5)-(7)). Bottom right: differences $\Delta z /\left(1+z_{\text {spec }}\right)$ as a function of the spectroscopic redshift $\left(X\right.$-axis). The shaded region illustrates the $3 \times \sigma_{\mathrm{NMAD}}$ uncertainty.

this range is mainly dominated by the light emitted by late-O and early-B stars (see e.g. Madau et al. 1998). In particular, we chose the SFR tracers proposed by Madau et al. (1998), which are based on stellar population models with exponentially declining SFRs, or $\tau$ models, and Salpeter (1955) IMF. Even though the SFR derived from luminosities at $1500 \AA$ and $\tau$ models is slightly less dependent on the duration of the star formation burst, the SFRs derived throughout this section were computed from the rest-frame luminosity at $2800 \AA, L_{2800 \AA}^{z=0}$. Formally,

$\mathrm{SFR}_{2800 \AA}=1.27 \times 10^{-28} \times L_{2800 \AA}^{z=0}$,

where $L_{2800 \AA}^{z=0}$ is in units of $\operatorname{ergs~s}^{-1} \mathrm{~Hz}^{-1}$, and $\mathrm{SFR}_{2800 \AA}$ in $M_{\odot} \mathrm{yr}^{-1}$. We note that Eq. (8) does not take dust effects into account. The choice of the SFR tracer at $2800 \AA$ is motivated by the observational wavelength-frame of ALHAMBRA because this starts at $\sim 3500 \AA$ (band F365W, full width at half maximum of $\sim 300 \AA$, and effective wavelength $\lambda_{\text {eff }}=3650 \AA$ ). Therefore, the SFR tracer based on the luminosity at $2800 \AA$ is only directly observed at redshift $z>0.25$, whereas for $1500 \AA$ at $z>1.3$, which would reduce our sample drastically. Actually, we are able to obtain a prediction of the luminosity at $2800 \AA$ at $z<0.25$, but this prediction would be an extrapolation of the SED fitting carried out by MUFFIT. For this section, we preferred a more conservative treatment in which the rest-frame flux at $2800 \AA$ must be included in the observational frame $(z \gtrsim 0.25)$.

The rest-frame luminosity $L_{2800 \AA}^{z=0}$, also free of dust attenuation, was calculated from the SED fitting results provided by MUFFIT. Similarly to the process of removing the dust effects on colours (Sect. 4.1), we rebuilt the combination of bestfitting models obtained during the Monte Carlo approach without extinction and for all the galaxies in ALHAMBRA. From this combination of models, we integrated the flux emitted in the rest-frame range $\lambda \lambda 2750-2850 \AA$ in order to compute $L_{2800 \AA}^{z=0}$ and subsequently $\mathrm{SFR}_{2800 \AA}$ via Eq. (8).

In order to characterise the range of $\mathrm{SFR}_{2800 \AA}$ values of star-forming galaxies, as well as their dependence on redshift and stellar mass, we studied the distribution of $\mathrm{SFR}_{2800 \AA}$ as a function of the stellar mass. When viewing the full sample of ALHAMBRA galaxies in the SFR vs. stellar-mass plane (see Fig. 5) we again differentiate between two groups of galaxies: star-forming and passive galaxies (e.g. Ilbert et al. 2010; 

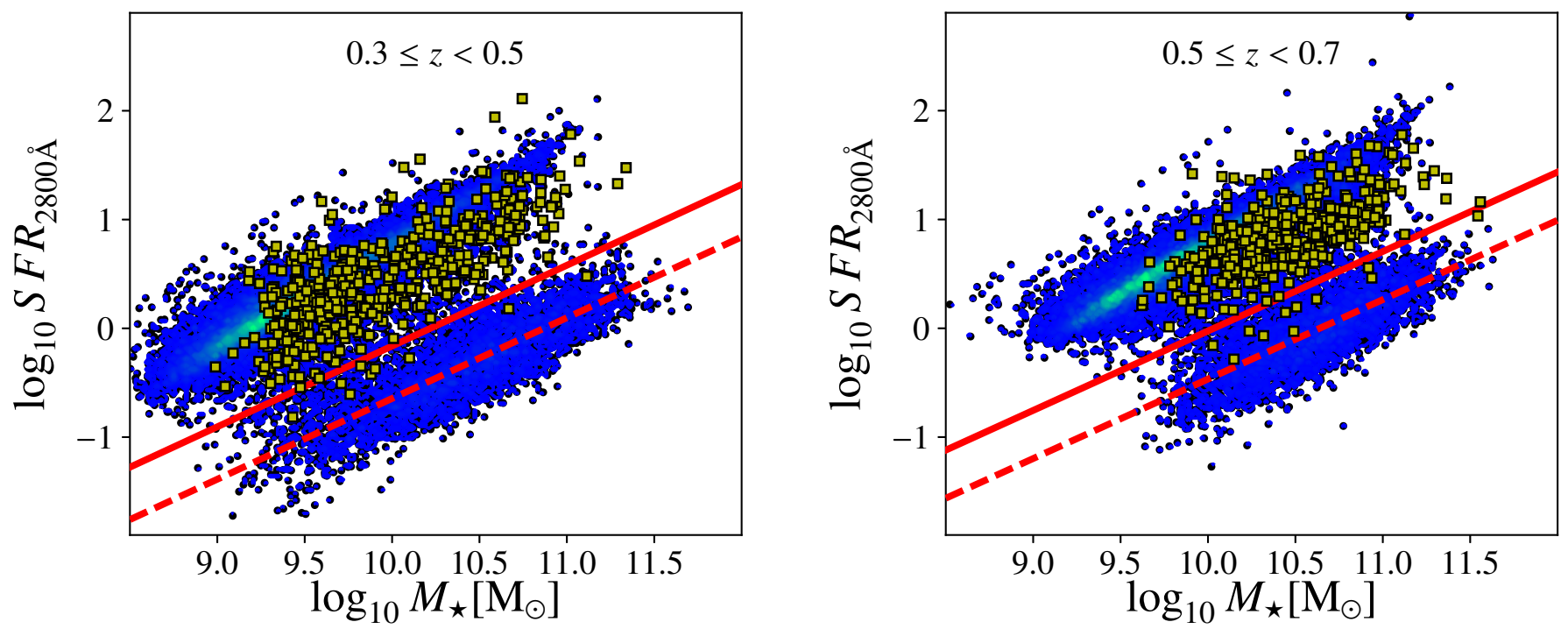

Fig. 5. Stellar mass $\left(X\right.$-axis) vs. star formation rate tracer $\operatorname{SFR}_{2800 \AA}(Y$-axis) defined by Madau et al. (1998, see Eq. (8)) of ALHAMBRA galaxies at redshift $0.3 \leq z<0.5$ (left panel) and $0.5 \leq z<0.7$ (right panel). High densities are presented in green, and lower densities in blue. The dashed

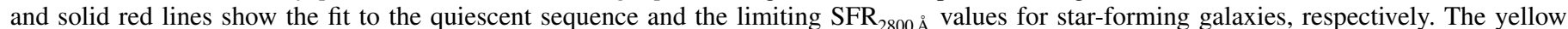
markers illustrate galaxies labelled as dusty star-forming galaxies by the rest-frame stellar mass-colour diagram corrected for extinction (MCDE).

Moustakas et al. 2013; Choi et al. 2014). This figure illustrates a tight correlation between the stellar mass and the SFR of a galaxy: the more massive the galaxy, the higher the SFR of the galaxy, independent of its spectral type (Daddi et al. 2007; Elbaz et al. 2007; Noeske et al. 2007; Moustakas et al. 2013). In order to separate quiescent and star-forming populations (i.e. setting

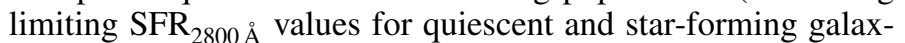
ies), we adapted the methodology detailed in Sect. 4.2, and repeated it. As a result, we obtain that the representative SFR values of quiescent galaxies, $\log _{10} \mathrm{SFR}_{2800 \AA}^{\mathrm{Q}}$, and their limiting values at a $3 \sigma$ level, $\log _{10} \mathrm{SFR}_{2800 \AA}^{\lim }$, are respectively expressed as

$$
\begin{aligned}
& \log _{10} \mathrm{SFR}_{2800 \AA}^{\mathrm{Q}}=0.75 \log _{10} M_{\star}-8.00 \\
& \log _{10} \mathrm{SFR}_{2800 \AA}^{\lim }=0.75\left(\log _{10} M_{\star}-10\right)-0.18(z-0.1)+0.11 .
\end{aligned}
$$

Our results point out that the correlation between $\log _{10} \mathrm{SFR}_{2800 \AA}$ and stellar mass is compatible with no evolution across cosmic time, and a relation of the form $\log _{10} \mathrm{SFR}_{2800 \AA} \propto$ $0.75 \log _{10} M_{\star}$ can be assumed for $\log _{10} \mathrm{SFR}_{2800 \AA}^{\mathrm{Q}}$. As in Sect. 4.2 , we took advantage of the MLE methodology presented in Appendix A to set the $3 \sigma$ limits of the distributions by the intrinsic scatter of SFR values, $\log _{10} \mathrm{SFR}_{2800 \AA}^{\text {rot }}$, defined as

$\log _{10} \mathrm{SFR}_{2800 \AA}^{\mathrm{rot}}=\log _{10} \mathrm{SFR}_{2800 \AA}-\log _{10} \mathrm{SFR}_{2800 \AA}^{\mathrm{Q}}$.

There are 828 DSF galaxies (number defined from the results obtained using BC03 SSP models) that were removed from the quiescent sample (complete in stellar mass and defined by the dust corrected $U V J$ diagram in Sect. 4.1) at redshift $0.3 \leq z \leq$ 1.1. Their $\mathrm{SFR}_{2800 \AA}$ measurements were compared with Eq. (10) to determine whether they present proper values of star-forming galaxies. We found that DSF galaxies mainly lie on the lower parts of the stellar mass-SFR plane of star-forming galaxies (see Fig. 5), that is, close to the SFR quiescent limit (Eq. (10)). Around $\sim 90 \%$ of the 828 DSF galaxies have $S^{2} R_{2800} \AA$ above Eq. (10), and consequently their SFR values are in the range of star-forming galaxies (see Fig. 5). This amount increases to $\sim 97 \%$ when we consider SFR errors ( $1 \sigma$ uncertainty level), which is almost the total of galaxies labelled as DSF galaxies. Moreover, we found that $\sim 98.2 \%$ of quiescent galaxies (defined by rest-frame colours in Sect. 4.1) exhibit a SFR below Eq. (10), which increases to $\sim 99.8 \%$ for a $1 \sigma$ uncertainty level. The same conclusion is obtained for EMILES SSP models.

These results additionally support MUFFIT predictions about DSF galaxies and the necessity of removing these galaxies, which comprise $\sim 10 \%$ of the sample when a $U V J$ selection is performed without removing the dust effects on colours (see details in Table 2). It should be noted that $\mathrm{SFR}_{2800 \AA}$ is also based on the SED fitting predictions carried out by MUFFIT because it is necessary to estimate the luminosity at $\sim 2800 \AA$ after removing dust effects, but it differs with respect to the selection process developed in Sect. 4.1, which is based on colour and not on a luminosity-based SFR tracer.

\section{Stellar populations of quiescent galaxies within the stellar mass-colour and UVJ colour-colour diagrams}

The empirical bimodality of galaxies in the stellar mass-colour and colour-colour diagrams is directly related to their stellar population properties (e.g. Bower et al. 1992; Gallazzi et al. 2006; Whitaker et al. 2010). This can be interpreted as a hint to explore whether within the quiescent sequence similar stellar population properties are constrained to lie on certain colour ranges. Colour predictions from models predict this, although there are also degeneracies and some stellar population properties are not observed in real galaxies. In this work we are in a privileged position because we are able to estimate the extinction, and thus to determine the main stellar population parameters that lead to the distribution of colours.

In this section, we explore the distribution of age, metallicity, extinction, and sSFR (Sects. 6.1-6.4, respectively) within the $U V J$ and stellar mass-colour diagrams (both corrected and noncorrected for extinction). A detailed analysis of the distributions 
Table 6. Parameters $\alpha, \beta, \gamma, \delta, \epsilon$, and $\zeta$ that more closely fit the distribution of stellar population parameters (extinction, age, metallicity, and sSFR) within the rest-frame $U V J$ colour-colour diagram corrected for extinction, see Eq. (12), for the SSP models of BC03 and EMILES (including BaSTI and Padova00 isochrones).

\begin{tabular}{|c|c|c|c|c|c|c|c|c|}
\hline & & $\alpha$ & $\beta$ & $\gamma$ & $\delta$ & $\epsilon$ & $\zeta$ & $\sigma_{\text {fit }}$ \\
\hline \multirow{4}{*}{ BC03 } & $A_{V}$ & $1.78 \pm 0.03$ & $2.59 \pm 0.06$ & $-2.70 \pm 0.07$ & $0.83 \pm 0.09$ & $-6.94 \pm 0.17$ & $6.55 \pm 0.14$ & 0.02 \\
\hline & $\log _{10}$ Age & $0.78 \pm 0.02$ & $1.93 \pm 0.03$ & $-1.88 \pm 0.04$ & $1.49 \pm 0.05$ & $-4.35 \pm 0.10$ & $12.34 \pm 0.08$ & 0.04 \\
\hline & {$[\mathrm{M} / \mathrm{H}]$} & $0.24 \pm 0.07$ & $-0.76 \pm 0.08$ & $-1.55 \pm 0.12$ & $3.48 \pm 0.15$ & $4.64 \pm 0.24$ & $-6.82 \pm 0.20$ & 0.09 \\
\hline & $\log _{10}$ sSFR & $0.42 \pm 0.06$ & $-1.20 \pm 0.14$ & $0.49 \pm 0.15$ & $-1.30 \pm 0.19$ & $1.39 \pm 0.41$ & $-9.41 \pm 0.30$ & 0.04 \\
\hline \multirow{4}{*}{ BaSTI } & $A_{V}$ & $1.25 \pm 0.05$ & $0.99 \pm 0.05$ & $-0.50 \pm 0.07$ & $-1.77 \pm 0.10$ & $-3.74 \pm 0.13$ & $5.13 \pm 0.11$ & 0.02 \\
\hline & $\log _{10}$ Age & $0.83 \pm 0.03$ & $2.23 \pm 0.03$ & $-2.79 \pm 0.04$ & $3.09 \pm 0.06$ & $-4.71 \pm 0.08$ & $12.15 \pm 0.07$ & 0.07 \\
\hline & {$[\mathrm{M} / \mathrm{H}]$} & $-0.37 \pm 0.15$ & $-0.56 \pm 0.08$ & $1.01 \pm 0.18$ & $2.35 \pm 0.24$ & $-0.45 \pm 0.21$ & $-1.34 \pm 0.21$ & 0.05 \\
\hline & $\log _{10}$ sSFR & $-0.83 \pm 0.07$ & $-0.79 \pm 0.09$ & $1.47 \pm 0.13$ & $-0.95 \pm 0.16$ & $-0.34 \pm 0.24$ & $-8.58 \pm 0.19$ & 0.07 \\
\hline \multirow{4}{*}{ Padova00 } & $A_{V}$ & $2.55 \pm 0.07$ & $2.70 \pm 0.07$ & $-3.60 \pm 0.11$ & $1.65 \pm 0.17$ & $-7.07 \pm 0.19$ & $6.44 \pm 0.16$ & 0.03 \\
\hline & $\log _{10}$ Age & $0.50 \pm 0.04$ & $2.05 \pm 0.03$ & $-2.74 \pm 0.07$ & $2.69 \pm 0.09$ & $-3.46 \pm 0.08$ & $11.12 \pm 0.07$ & 0.04 \\
\hline & {$[\mathrm{M} / \mathrm{H}]$} & $-6.23 \pm 0.13$ & $-0.12 \pm 0.07$ & $6.15 \pm 0.15$ & $4.66 \pm 0.25$ & $-6.94 \pm 0.22$ & $3.10 \pm 0.21$ & 0.06 \\
\hline & $\log _{10}$ sSFR & $-0.91 \pm 0.12$ & $-0.97 \pm 0.13$ & $2.59 \pm 0.22$ & $-1.52 \pm 0.28$ & $-1.94 \pm 0.31$ & $-6.50 \pm 0.23$ & 0.04 \\
\hline
\end{tabular}

Notes. The last column illustrates the average data scatter with respect to the plane fit (Eq. (12)).

Table 7. Same as Table 6, but for the rest-frame stellar mass-colour diagram corrected for extinction (MCDE).

\begin{tabular}{|c|c|c|c|c|c|c|c|c|}
\hline & & $\alpha$ & $\beta$ & $\gamma$ & $\delta$ & $\epsilon$ & $\zeta$ & $\sigma_{\text {fit }}$ \\
\hline \multirow{4}{*}{ ВC03 } & $A_{1}$ & $-0.037 \pm 0.003$ & $1.16 \pm 0.06$ & $-0.05 \pm 0.02$ & $0.90 \pm 0.06$ & $-4.44 \pm 0.22$ & $-0.03 \pm 0.35$ & 0.03 \\
\hline & $\log _{10}$ Age & $0.054 \pm 0.001$ & $0.56 \pm 0.02$ & $0.06 \pm 0.01$ & $-1.26 \pm 0.02$ & $-2.20 \pm 0.08$ & $18.07 \pm 0.14$ & 0.04 \\
\hline & {$[\mathrm{M} / \mathrm{H}]$} & $-0.058 \pm 0.005$ & $-0.49 \pm 0.08$ & $-0.40 \pm 0.04$ & $2.08 \pm 0.09$ & $7.29 \pm 0.33$ & $-19.37 \pm 0.59$ & 0.07 \\
\hline & $\log _{10}$ SSFR & $-0.037 \pm 0.007$ & $-0.21 \pm 0.11$ & $0.20 \pm 0.05$ & $0.46 \pm 0.12$ & $-3.55 \pm 0.40$ & $-8.28 \pm 0.66$ & 0.02 \\
\hline \multirow{4}{*}{ BaSTI } & $A_{V}$ & $-0.034 \pm 0.003$ & $0.80 \pm 0.04$ & $0.05 \pm 0.01$ & $0.68 \pm 0.05$ & $-4.16 \pm 0.18$ & $0.84 \pm 0.34$ & 0.02 \\
\hline & $\log _{10}$ Age & $0.012 \pm 0.001$ & $0.35 \pm 0.01$ & $0.02 \pm 0.01$ & $-0.35 \pm 0.02$ & $-1.30 \pm 0.06$ & $12.93 \pm 0.10$ & 0.05 \\
\hline & {$[\mathrm{M} / \mathrm{H}]$} & $-0.110 \pm 0.002$ & $0.71 \pm 0.02$ & $-0.21 \pm 0.01$ & $2.95 \pm 0.04$ & $0.05 \pm 0.11$ & $-17.51 \pm 0.26$ & 0.06 \\
\hline & $\log _{10}$ SSFR & $-0.021 \pm 0.005$ & $0.10 \pm 0.07$ & $0.05 \pm 0.03$ & $0.37 \pm 0.10$ & $-2.58 \pm 0.26$ & $-9.19 \pm 0.54$ & 0.03 \\
\hline \multirow{4}{*}{ Padova00 } & $A_{V}$ & $-0.033 \pm 0.003$ & $0.98 \pm 0.06$ & $0.05 \pm$ & $0.64 \pm 0.06$ & $-4.97 \pm 0.21$ & 1.82 & 0.02 \\
\hline & $\log _{10}$ Age & $0.037 \pm 0.001$ & $0.31 \pm 0.02$ & $0.00 \pm 0.01$ & $-0.85 \pm 0.02$ & $-0.72 \pm 0.07$ & $14.94 \pm 0.13$ & 0.03 \\
\hline & {$[\mathrm{M} / \mathrm{H}]$} & $-0.086 \pm 0.003$ & $0.93 \pm 0.04$ & $-0.40 \pm 0.02$ & $2.75 \pm 0.05$ & $1.57 \pm 0.15$ & $-18.01 \pm 0.29$ & 0.04 \\
\hline & $\log _{10}$ SSFR & $-0.041 \pm 0.007$ & $0.14 \pm 0.11$ & $0.14 \pm 0.05$ & $0.68 \pm 0.12$ & $-4.05 \pm 0.34$ & $-9.27 \pm 0.66$ & 0.02 \\
\hline
\end{tabular}

of stellar population properties of quiescent galaxies and their dependence on stellar mass, and their evolution with redshift, is beyond the scope of this work. We refer readers to the next papers in this series (Díaz-García et al. 2019a,b) where we will explore this topic in depth.

In all the cases instead of plotting the individual measurements, which include uncertainties, we carried out a bidimensional and locally weighted regression method called LOESS (Cleveland 1979; Cleveland \& Devlin 1988). This methodology finds a non-parametric plane to reveal trends on both the stellar mass-colour and $U V J$ diagrams through the distribution of points (stellar population parameters for this study) minimising the uncertainty effects on the diagrams by a regression technique. We note that the LOESS method does not assume a model or function to fit the distribution of stellar population properties to a plane, which in practice is similar to estimating average values of parameters across these diagrams (for further details, see Cleveland 1979; Cleveland \& Devlin 1988). Specifically, we used the LOESS implementation for the Python language ${ }^{13}$ (Cappellari et al. 2013), where the regularisation factor or smoothness is set to $f=0.6$ and a local linear approximation is assumed; this method yielded satisfactory results in previous works (e.g. Cappellari et al. 2013; McDermid et al. 2015). The uncertainties on stellar mass, extinction, age, metallicity, and

\footnotetext{
${ }^{13}$ http://www-astro.physics.ox . ac.uk/ mxc/software/
}

sSFR of individual galaxies were also taken into account during the regression process.

In addition, we carried out a bi-dimensional fit of the distributions obtained from the LOESS method to quantify the distributions of stellar population properties within the restframe $U V J$ and MCDE diagrams (both corrected for extinction). The distributions of extinction, age, metallicity, and sSFR were quadratically fit to a plane using

$\mathcal{P}=\alpha \cdot \mathcal{X}^{2}+\beta \cdot \mathcal{Y}^{2}+\gamma \cdot \mathcal{X} \cdot \boldsymbol{Y}+\delta \cdot \mathcal{X}+\epsilon \cdot \boldsymbol{Y}+\zeta$,

where $\mathcal{P}$ is the stellar population property (extinction, age, metallicity, and sSFR), $\mathcal{X}$ is either the intrinsic colour $\left(m_{\mathrm{F} 551}-\right.$ $J)_{\text {int }}$ or the stellar mass in dex units (for the dust corrected $U V J$ or MCDE diagrams, respectively), $\mathcal{Y}$ is the colour $\left(m_{\mathrm{F} 365}-\right.$ $\left.m_{\mathrm{F} 551}\right)_{\mathrm{int}}$ corrected for extinction, and $\alpha, \beta, \gamma, \delta, \epsilon$, and $\zeta$ are the parameters of the quadratically fit. The values of these parameters for the $U V J$ and MCDE diagrams are provided in Tables 6 and 7 , respectively. As a result, average differences between these planes and the distribution of points are almost null and the average scatter $\left(\sigma_{\text {fit }}\right)$ is below $\sim 0.05$ dex in most of the cases (see Tables 6 and 7). Independently of the SSP model set, the stellar population properties exhibiting lower $\sigma_{\text {fit }}$ values correspond to extinction and SSFR with values of $\sigma_{\text {fit }} \sim 0.02 \mathrm{dex}$, whereas the higher values are related to metallicity. We note that this parametrisation of the distributions may be very useful 
Table 8. Spearman correlation coefficients between the dust-corrected colours $\left(m_{\mathrm{F} 365}-m_{\mathrm{F} 551}\right)_{\text {int }}$ and $\left(m_{\mathrm{F} 551}-J\right)_{\mathrm{int}}$ and stellar population parameters $\left(\rho_{U V}\right.$ and $\rho_{V J}$, respectively).

\begin{tabular}{|c|c|c|c|c|c|c|}
\hline & \multicolumn{2}{|c|}{$\mathrm{BC} 03$} & \multicolumn{2}{|c|}{ BaSTI } & \multicolumn{2}{|c|}{ Padova00 } \\
\hline & $\rho_{\mathrm{UV}}$ & $\rho_{\mathrm{VJ}}$ & $\rho_{\mathrm{UV}}$ & $\rho_{\mathrm{VJ}}$ & $\rho_{\mathrm{UV}}$ & $\rho_{\mathrm{VJ}}$ \\
\hline$A_{V}$ & -0.90 & -0.80 & -0.95 & -0.77 & -0.91 & -0.67 \\
\hline $\log _{10}$ Age & 0.61 & 0.23 & 0.30 & 0.17 & 0.46 & 0.07 \\
\hline$[\mathrm{M} / \mathrm{H}]$ & 0.68 & 0.84 & 0.24 & 0.75 & 0.46 & 0.80 \\
\hline $\log _{10}$ sSFR & -0.98 & -0.55 & -0.98 & -0.75 & -0.96 & -0.67 \\
\hline
\end{tabular}

for future works to estimate these parameters only knowing the positions of quiescent galaxies within these diagrams. In addition, we quantified the correlations between the stellar population properties (extinction, age, metallicity, and sSFR) and both the $\left(m_{\mathrm{F} 365}-m_{\mathrm{F} 551}\right)_{\text {int }}$ and $\left(m_{\mathrm{F} 551}-J\right)_{\text {int }}$ colours via a Spearman correlation coefficient ( $\rho_{U V}$ and $\rho_{V J}$, respectively, see Table 8). This coefficient presents values in the range $-1 \leq \rho_{U V, V J} \leq 1$, where 1 is total positive correlation, 0 is no correlation, and -1 total anticorrelation.

In Figs. 6 and 7, we illustrate the distribution of stellar population parameters (non-dust corrected and intrinsic colours, respectively) for quiescent galaxies on the rest-frame $U V J$ diagram for BC03 SSP models. To guide the eye, we also plot the typical selection of quiescent galaxies (illustrated in Fig. 7, see Eq. (1) in this work and Moresco et al. 2013). Interestingly, the only selection criterion $\left(m_{\mathrm{F} 365}-m_{\mathrm{F} 551}\right)_{\text {int }} \geq 1.5$ (see Fig. 6) provides a quiescent sample whose observed colour ranges are well delimited by Eq. (1). In our sample of quiescent galaxies, we only find $1 \%$ of galaxies with $m_{\mathrm{F} 365}-$ $m_{\mathrm{F} 551}$ and $m_{\mathrm{F} 551}-J$ colours outside the box delimited by Eq. (1).

We illustrate the stellar population parameters of the quiescent galaxies selected by the MCDE (see Eq. (3) and Table 3) in Figs. 8 and 9 (corrected and non-corrected for reddening, respectively). The analysis of the distribution of stellar population properties of quiescent galaxies in the stellar mass-colour diagram complements and makes the interpretation of the analysis within the $U V J$-diagram easier. We detail the main features of the distribution of stellar population parameters below, which were equally obtained for the three SSP model sets used in this work (BC03 and EMILES, the latter including BaSTI and Padova00 isochrones).

\subsection{Distribution of extinction within the UVJ and stellar mass-colour diagrams}

In Figs. 6 and 7, we see that fewer massive quiescent galaxies tend to populate the bluest parts of the $U V J$-diagram, whereas at increasing red colours $\left(m_{\mathrm{F} 365}-m_{\mathrm{F} 551}\right)_{\text {int }}$ and $\left(m_{\mathrm{F} 551}-J\right)_{\text {int }}$ quiescent galaxies continuously present larger stellar masses. However, the most massive galaxies are concentrated in the upper part of the $U V J$-diagram at decreasing redshift, $\left(m_{\mathrm{F} 365}-m_{\mathrm{F} 551}\right)_{\text {int }} \sim$ 2.0. Nevertheless, most massive galaxies are scattered over a wider $\left(m_{\mathrm{F} 365}-m_{\mathrm{F} 551}\right)_{\text {int }}$ range when we explore the highest redshift panels, $1.5 \leq\left(m_{\mathrm{F} 365}-m_{\mathrm{F} 551}\right)_{\text {int }} \leq 2.0$. This has been extensively observed in the last years (e.g. Bower et al. 1992; Kauffmann et al. 2003; Gallazzi et al. 2005; Baldry et al. 2006; Peng et al. 2010). We note that each panel comprises different stellar mass ranges, as indicated in the upper labels and according to Fig. 3, hence the less massive galaxies are only present in the local redshift bins.
From our SED fitting analysis, we see in Fig. 6 that the whole sample shows an expected low dust content $(96 \%$ of galaxies present $\left.A_{V} \leq 0.6\right)$, where the more obscured quiescent galaxies lie on the bluer $\left(m_{\mathrm{F} 365}-m_{\mathrm{F} 551}\right)_{\mathrm{int}}$ and $\left(m_{\mathrm{F} 551}-J\right)_{\text {int }}$ intrinsic colour regions of the diagram. On the other hand, if dust effects on the colours are not corrected (observational rest-frame colours, Fig. 7), dusty galaxies populate the red parts of the diagram. Moreover, extinction is anticorrelated with both $\left(m_{\mathrm{F} 365}-m_{\mathrm{F} 551}\right)_{\text {int }}$ and $\left(m_{\mathrm{F} 551}-J\right)_{\text {int }}$ dust-corrected colours, with Spearman correlation coefficients of $\rho_{U V} \sim-0.92$ and $\rho_{V J} \sim-0.75$, respectively (see Table 8). As a sanity check, the colour changes owing to a dust reddening case with $A_{V}=0.5$ and $R_{V}=3.1$ and the extinction law of Fitzpatrick $(1999)$ are $\Delta\left(m_{\mathrm{F} 365}-m_{\mathrm{F} 551}\right) \sim 0.28$ and $\Delta\left(m_{\mathrm{F} 551}-J\right) \sim 0.29$ (illustrated in Figs. 6 and 7 ). Our results are in good agreement with the colour changes predicted by this extinction law and the observed change in the galaxy distribution between Figs. 6 and 7 at any redshift. We also note that the extinction values provided by MUFFIT are properly decoupled and are not significantly affected by degeneracies with the rest of stellar population parameters (age and metallicity). Otherwise, the extinction distribution in the $U V J$ diagram would be randomly distributed or it would show other colour dependences.

It is clear from Fig. 8 that the quiescent galaxies exhibiting the larger extinction are those that also present the bluest intrinsic colours. It is interesting that there is a clear correlation of extinction with the intrinsic colour $\left(m_{\mathrm{F} 365}-m_{\mathrm{F} 551}\right)_{\text {int }}$, but also with the stellar mass of the galaxy since $z \sim 1$. At fixed intrinsic colour $\left(m_{\mathrm{F} 365}-m_{\mathrm{F} 551}\right)_{\text {int }}$, the more massive the quiescent galaxy, the greater the reddening by dust. Whilst this correlation exists, discrepancies between extinction values are not very remarkable, since extinction is typically low for the quiescent population.

When we explore the same diagram but without correcting colours for extinction (see Fig. 9), the reddest galaxies are those with the greatest dust content (for $A_{V} \gtrsim 0.4, m_{\mathrm{F} 365}-m_{\mathrm{F} 551} \gtrsim 2$ ). Thus, at fixed colour $m_{\mathrm{F} 365}-m_{\mathrm{F} 551}$, more massive galaxies are less dust-reddened than less massive ones.

\subsection{Ages of quiescent galaxies within the UVJ and stellar mass-colour diagrams}

Older quiescent galaxies in the sample are concentrated in the upper part of the $U V J$ diagram (details in Figs. 6 and 7), and they therefore tend to populate the intrinsic redder colours $\left(m_{\mathrm{F} 365}-\right.$ $\left.m_{\mathrm{F} 551}\right)_{\text {int }}$. Likewise, young quiescent galaxies lie on the bluest colours in concordance with the less massive systems in the sample. In Fig. 6, we see that the variety of ages presented by quiescent galaxies is linked to the scatter of $\left(m_{\mathrm{F} 365}-m_{\mathrm{F} 551}\right)_{\text {int }}$ partly (correlation coefficient of $\rho_{U V} \sim 0.45$, see Table 8 ), but not fully linked to this colour. In the rest-frame $U V J$ diagram, we also find a non-negligible dependence of the age on $\left(m_{\mathrm{F} 551}-J\right)_{\text {int }}$, although milder than the other intrinsic colour (correlation coefficient of $\left.\rho_{\mathrm{VJ}} \sim 0.15\right)$. For the EMILES and BaSTI isochrones the age and $\left(m_{\mathrm{F} 551}-J\right)_{\text {int }}$ colour are not correlated with a value of $\rho_{V J} \sim-0.04$ (see Table 8). Consequently, our results match with previous findings (e.g. Whitaker et al. 2010) in which the $\left(m_{\mathrm{F} 365}-m_{\mathrm{F} 551}\right)_{\text {int }}$ colour is scattered by the ages in the quiescent population. This is not surprising because the $\left(m_{\mathrm{F} 365}-m_{\mathrm{F} 551}\right)_{\text {int }}$ colour ranges in the $4000 \AA$ break, which is sensitive to age (e.g. Bruzual 1983; Balogh et al. 1999), even though this also degenerates with the metallicity (Worthey et al. 1994; Peletier 2013). On the contrary, the non-dust corrected $U V J$ diagram (see Fig. 7) shows that the observed colour $m_{\mathrm{F} 365}-m_{\mathrm{F} 551}$ is not driven by the age. As 


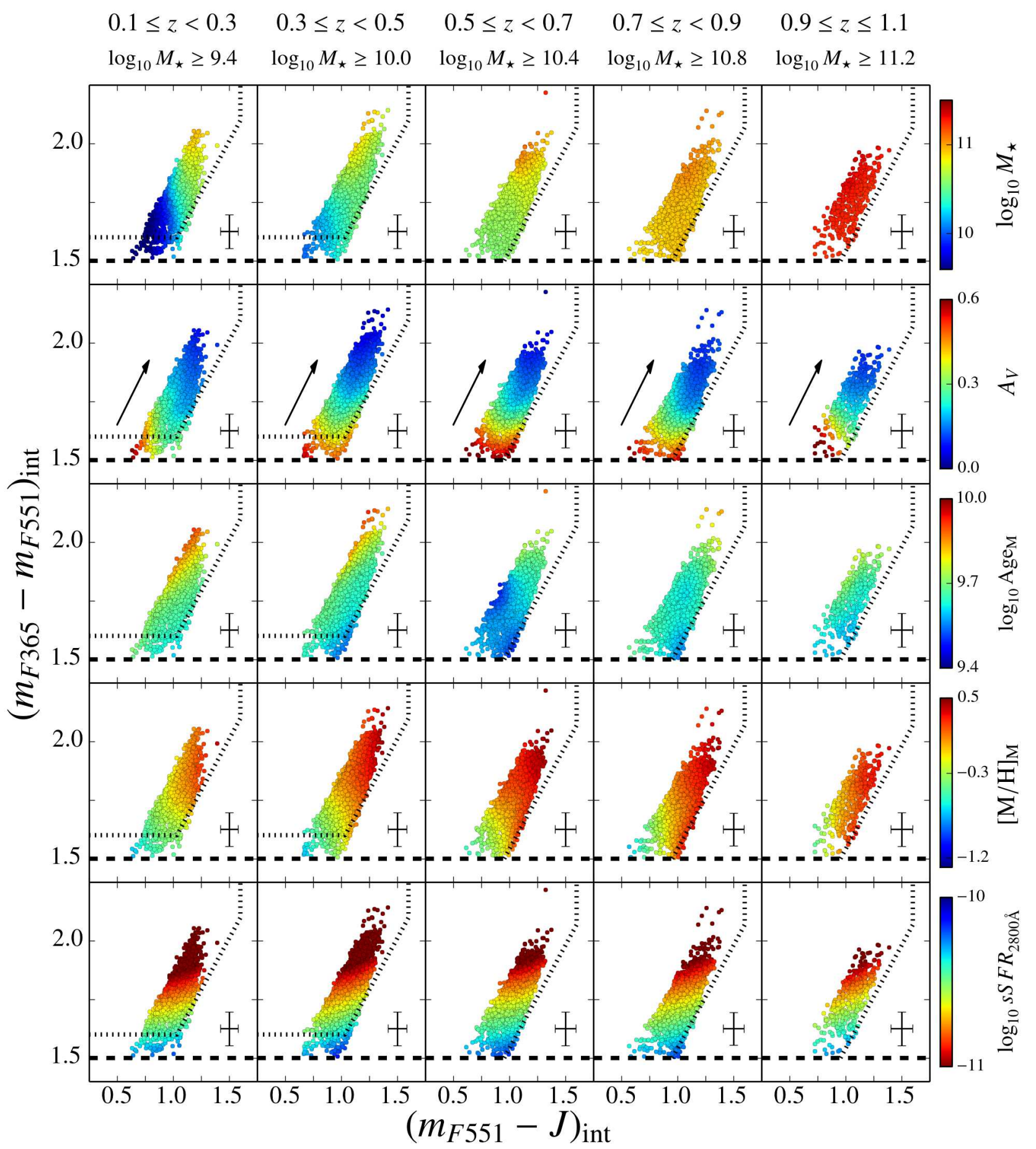

Fig. 6. Stellar population parameters in the rest-frame $U V J$ diagram. At different redshift bins, we present the intrinsic colours $\left(m_{\mathrm{F} 551}-J\right)_{\text {int }}(X-$ axis) and $\left(m_{\mathrm{F} 365}-m_{\mathrm{F} 551}\right)_{\text {int }}(Y$-axis) after correcting for extinction for the mass complete sample of quiescent galaxies (see stellar mass completeness at the top). The different stellar population parameters are colour-coded as a function of their values and obtained using BC03 SSP models (see the colour bars to the right of each row). From top to bottom: stellar mass, extinction, mass-weighted age and metallicity, and specific star formation rate. All the parameters were spatially averaged through a LOESS method. Black crosses illustrate the median uncertainties in both $\left(m_{\mathrm{F} 551}-J\right)_{\text {int }}$ and $\left(m_{\mathrm{F} 365}-m_{\mathrm{F} 551}\right)_{\mathrm{int}}$ intrinsic colours. The dotted black line encloses the rest-frame colour ranges assumed for selecting quiescent galaxies in Moresco et al. (2013, see Eq. (1)), while the dashed line illustrates our colour limit for selecting quiescent galaxies $\left(m_{\mathrm{F} 365}-m_{\mathrm{F} 551}\right)_{\text {int }}>1.5$. We illustrate the colour variations owing to a reddening of $A_{V}=0.5$ (black arrow), assuming the extinction law of Fitzpatrick (1999).

illustrated by Fig. 7, the trends of age with $m_{\mathrm{F} 365}-m_{\mathrm{F} 551}$ are not as clear as in the intrinsic colours, or they look inverted. Therefore, extinction also plays an important role in this aspect by masking and blurring the relation between $\left(m_{\mathrm{F} 365}-m_{\mathrm{F} 551}\right)_{\text {int }}$ and age.

In Fig. 8, we find that the most massive quiescent galaxies are also the oldest and intrinsically the reddest. This agrees with the "downsizing" scenario (Cowie et al. 1996), where the more massive galaxies were formed in earlier epochs of the Universe with respect to their less massive counterparts. These very evolved galaxies also present very low extinction. When studying the distribution of age in the stellar mass-colour diagram without correcting colours for dust (Fig. 9), the dependence 


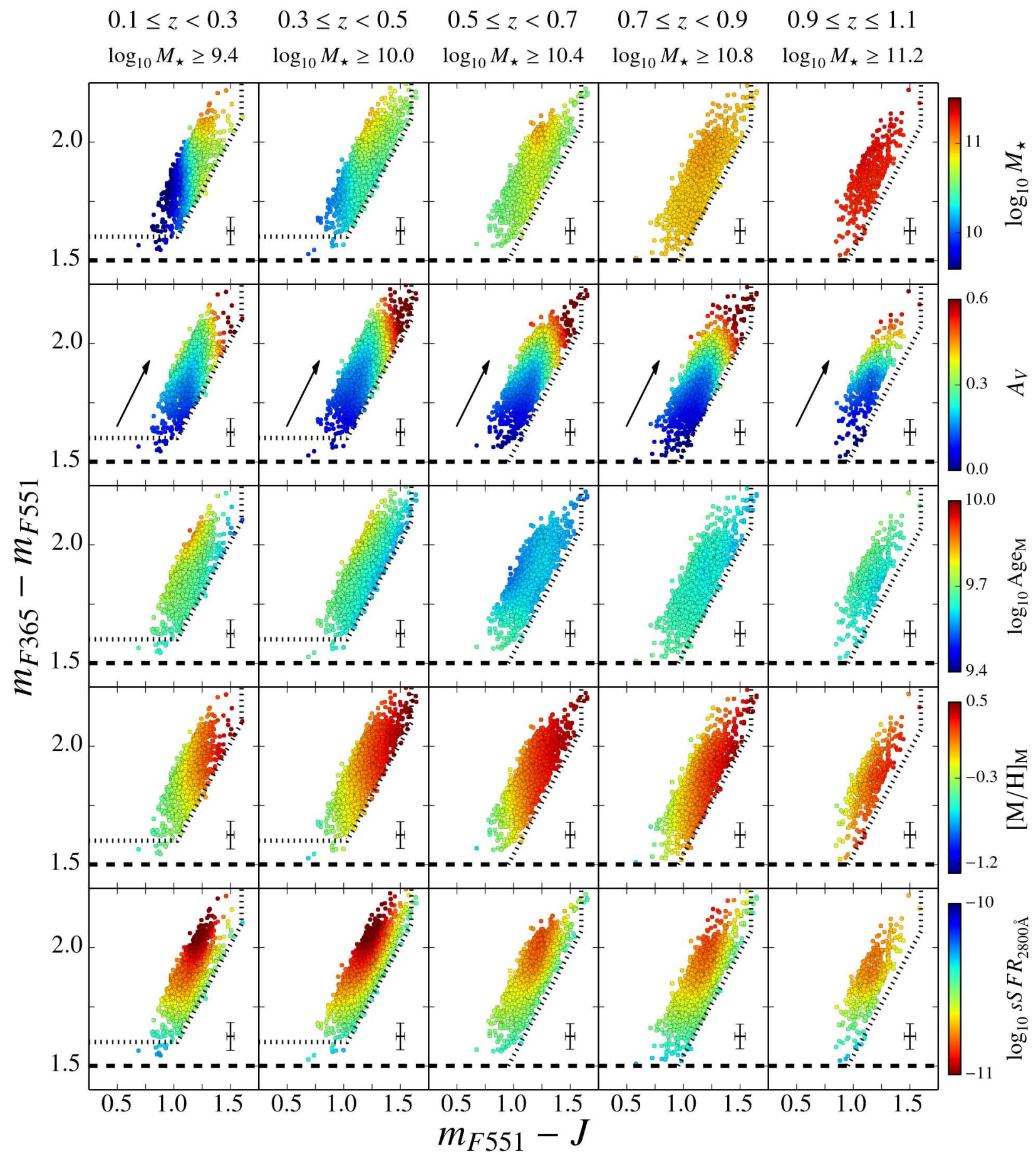

Fig. 7. Same as Fig. 6, but for the rest-frame colours $m_{\mathrm{F} 551}-J$ ( $X$-axis) and $m_{\mathrm{F} 365}-m_{\mathrm{F} 551}(Y$-axis), meaning non-dust corrected colours.

between $m_{\mathrm{F} 365}-m_{\mathrm{F} 551}$ and age is softly blurred, although the most massive and reddest $\left(m_{\mathrm{F} 365}-m_{\mathrm{F} 551} \geq 1.8\right)$ are also the oldest.

\subsection{Distributions of metallicity}

Exploring the metallicity distribution in the $U V J$ diagram (see Fig. 6) we find that there is a tight correlation between metallicity and the intrinsic $\left(m_{\mathrm{F} 551}-J\right)_{\text {int }}$ colour, where the correlation coefficient is $\rho_{V J} \sim 0.85$ (see Table 8). There is a clear trend in which higher metallicity content $\left([\mathrm{M} / \mathrm{H}]_{\mathrm{M}}>0.1 \mathrm{dex}\right)$ is present with redder $\left(m_{\mathrm{F} 551}-J\right)_{\text {int }}>1.1$ colours. Hence, the most metal-rich quiescent galaxies lie on the right-hand side of the $U V J$ diagram, and this trend is maintained at least up to redshift $z \sim 1$. For a fixed $\left(m_{\mathrm{F} 551}-J\right)_{\mathrm{int}}$ colour, the influence of the metal content on the $\left(m_{\mathrm{F} 365}-m_{\mathrm{F} 551}\right)_{\text {int }}$ is almost negligible in a wide range of the colour $\left(m_{\mathrm{F} 551}-J\right)_{\mathrm{int}}$, butitis not null. Whilst the age distribution is distorted by extinction, the metallicity trend with the colour $m_{\mathrm{F} 551}-J$ is still prominent (see Fig. 7). The main extinction effect over metallicity distribution is that at the high-redshift panels (or the most massive galaxies, $\log _{10} M_{\star}>10.8 \mathrm{dex}$ ), the metallicity exhibits a more remarkable dependence on the $m_{\mathrm{F} 365}-m_{\mathrm{F} 551}$ colour with respect to its intrinsic counterpart (see Fig. 6) and it is substantially less affected than the age. 


\section{$0.1 \leq z<0.3 \quad 0.3 \leq z<0.5 \quad 0.5 \leq z<0.7 \quad 0.7 \leq z<0.9 \quad 0.9 \leq z \leq 1.1$}

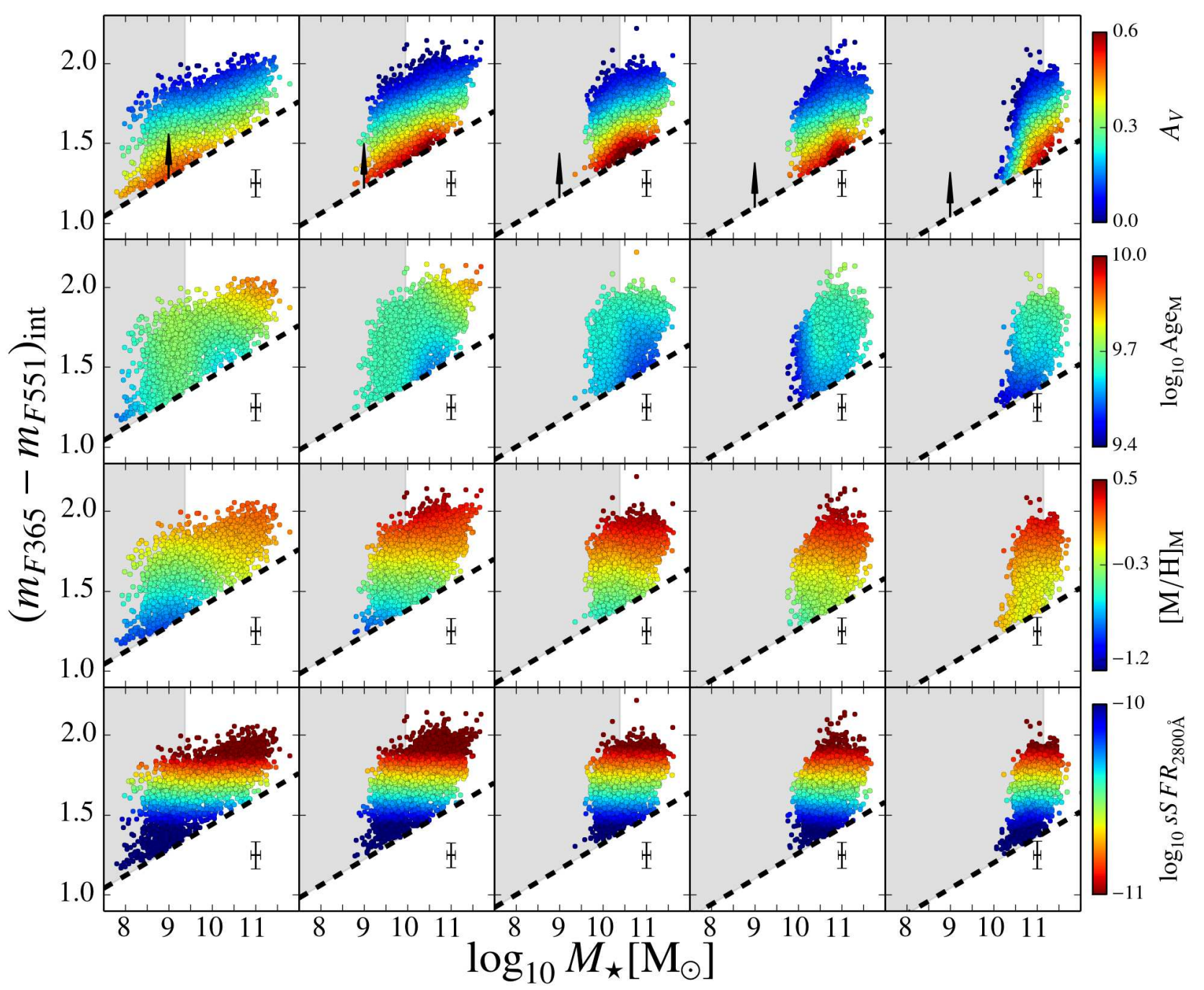

Fig. 8. Stellar population parameters in the rest-frame stellar mass-colour diagram. At different redshift bins, we present the stellar mass $(X$-axis) and intrinsic colour $\left(m_{\mathrm{F} 365}-m_{\mathrm{F} 551}\right)_{\mathrm{int}}(Y$-axis $)$ after correcting for extinction. The stellar population parameters are colour-coded according to their values using BC03 SSP models (see colour bars). From top to bottom: extinction, mass-weighted age and metallicity, and specific star formation rate. All the parameters were spatially averaged through a LOESS method. Black crosses illustrate the median uncertainties in both stellar mass and $\left(m_{\mathrm{F} 365}-m_{\mathrm{F} 551}\right)_{\mathrm{int}}$ intrinsic colour. The dashed line illustrates the colour limit for selecting quiescent galaxies in the MCDE, see Eq. (3) and Table 3, for this work. The shaded regions show the stellar mass range in which our quiescent sample is not complete in stellar mass. We illustrate the colour variations owing to a reddening of $A_{V}=0.5$ (black arrow), assuming the extinction law of Fitzpatrick (1999).

In Fig. 8, we observe that the stellar mass-metallicity relation is also present, reinforcing the reliability of the stellar population properties obtained by MUFFIT using ALHAMBRA photometry of galaxies. The more massive the galaxy, the more metal rich it is. This is usually referred to as the stellar massmetallicity relation (MZR), which has been studied previously (Trager et al. 2000; Tremonti et al. 2004; Gallazzi et al. 2005; Panter et al. 2008; González Delgado et al. 2014a). When we focus on the diagram without the dust correction (see Fig. 9), metallicity values populate well-defined regions of the stellar mass-colour diagrams.

\section{4. sSFR within the UVJ and stellar mass-colour diagrams}

Like the other stellar population properties explored in this work, lower and higher sSFRs of quiescent galaxies populate different colour ranges in dust-corrected $U V J$ diagrams (details in Fig. 6). Our results indicate that the lowest sSFRs lie on the upper parts of this diagram, independently of the stellar mass and redshift. This is not surprising because at increasing stellar mass, quiescent galaxies exhibit lower sSFR and they are also redder. The correlation between SSFR and the intrinsic colour $\left(m_{\mathrm{F} 365}-m_{\mathrm{F} 551}\right)_{\text {int }}$ is really remarkable, with a value $\rho_{U V}$ close to -1 (see Table 8). Furthermore, there is also an anticorrelation with $\left(m_{\mathrm{F} 551}-J\right)_{\text {int }}$, although milder $\left(\rho_{V J} \sim-0.65\right)$. It should be noted that sSFRs were obtained from the luminosity at $2800 \AA$, Eq. (8), but we find evidence to propose the intrinsic colour $\left(m_{\mathrm{F} 365}-m_{\mathrm{F} 551}\right)_{\text {int }}$ as an alternative sSFR tracer, at least for quiescent galaxies. Nevertheless, this tracer should be treated carefully. When there is no star formation, Eq. (8) provides non-null SFRs because there is a non-null continuum at $2800 \AA$, although these values can be treated as upper sSFR limits. In Fig. 7, we find that the correlation between $\left(m_{\mathrm{F} 365}-m_{\mathrm{F} 551}\right)$ and sSFR is not as clear as the correlation with intrinsic colour. However, certain range colours present predominantly lower sSFRs. The lowest sSFR values lie on the upper parts of classic $U V J$ diagrams 


\section{$0.1 \leq z<0.3 \quad 0.3 \leq z<0.5 \quad 0.5 \leq z<0.7 \quad 0.7 \leq z<0.9 \quad 0.9 \leq z \leq 1.1$}

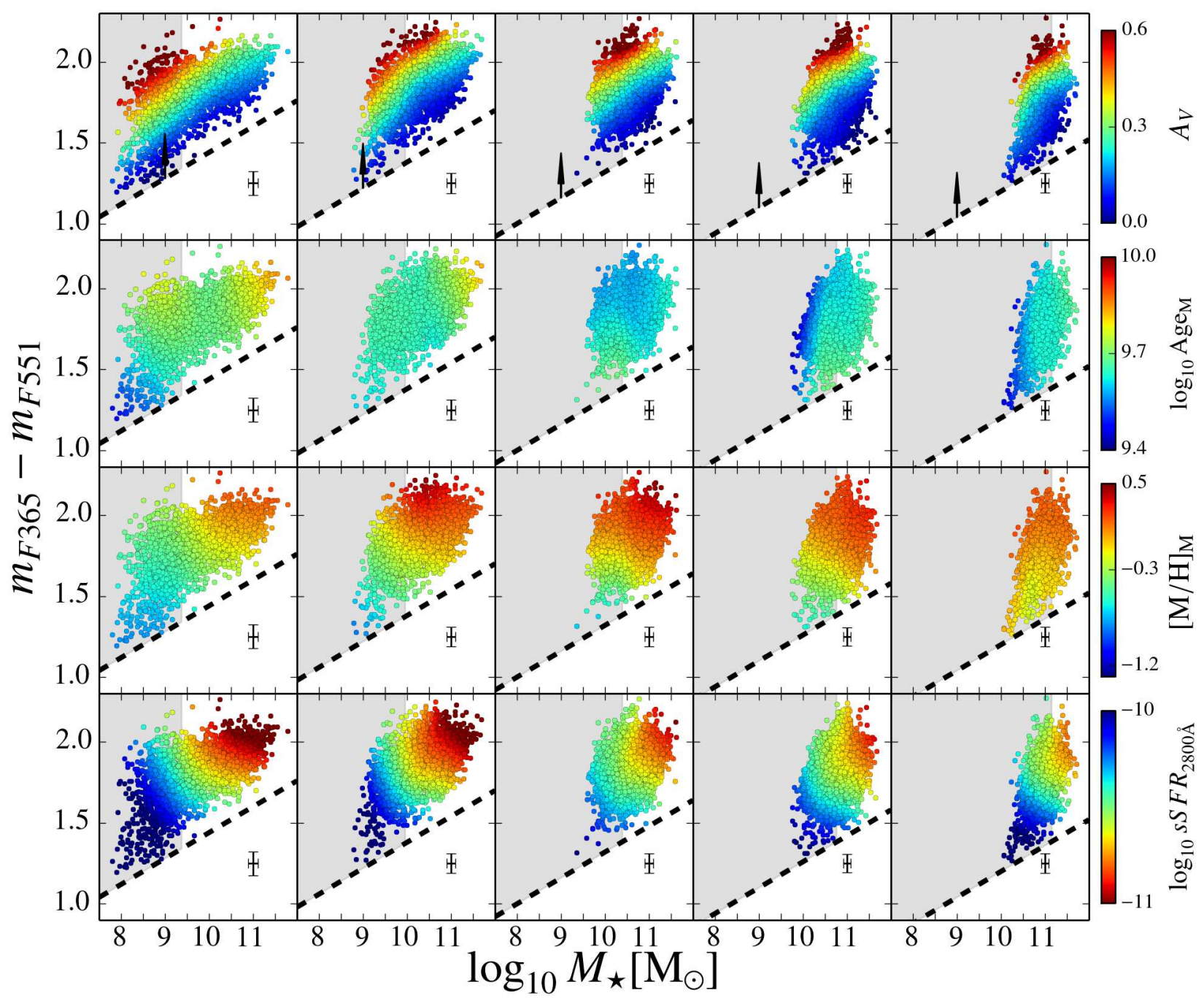

Fig. 9. Same as Fig. 8, but for the rest-frame colour $m_{\mathrm{F} 365}-m_{\mathrm{F} 551}$ ( $X$-axis, non-dust corrected colour).

(non-dust corrected), whereas the highest values present bluer colours. This matches with the distribution of stellar mass in these diagrams (see Fig. 7), which is not surprising. As above, extinction displaces galaxies within these diagrams, which means that dust-obscured quiescent galaxies with higher sSFRs also lie on the upper parts of $U V J$ diagrams.

The sSFR values of quiescent galaxies also present welldefined loci in the MCDE (see lower panels in Fig. 8). At increasing stellar mass, lower sSFR values are obtained. It is interesting that the intrinsic colour $\left(m_{\mathrm{F} 365}-m_{\mathrm{F} 551}\right)_{\text {int }}$ can be interpreted as an alternative sSFR tracer, also motivated by the high anticorrelation that presents with this colour, $\rho_{U V} \sim-1$ (see Table 8). However, to constrain the sSFR of quiescent galaxies using $m_{\mathrm{F} 365}-m_{\mathrm{F} 551}$, it is necessary to include the stellar mass. As in previous stellar population properties, extinction is an important parameter that dilutes the correlations and trends revealed in this work (see Fig. 9).

Although it is well known that the use of different stellar population models yields different quantitative results, we find a great qualitative agreement between all the predictions obtained using the BC03 and EMILES SSP models. All the conclusions drawn in this section can be extrapolated when EMILES SSP models (both BaSTI and Padova00 isochrones) are used.

\section{Discussion}

\subsection{New insights into the green valley}

Dust corrections play an important role in understanding how quiescent galaxies are distributed across $U V J$ diagrams as a function of their parameters: stellar mass, age, metallicity, and extinction. One of the most important results in this paper is that the green valley is largely populated by DSF galaxies $(\sim 65 \%)$, and therefore the number of galaxies in the "real" green valley is much lower than previously claimed. This implies that the transition of galaxies from the blue cloud to the red sequence, and hence the related mechanisms for quenching, should be much more efficient and faster than previously considered.

Any successful mechanisms proposed for shutting down the star formation should account for a less populated green valley and a shorter transition timescale. For instance, the common presence of AGNs in galaxies with intermediate hostgalaxy colours at $z \lesssim 1$ (Nandra et al. 2007; Bundy et al. 2008; Georgakakis et al. 2008; Silverman et al. 2008; Hickox et al. 2009; Schawinski et al. 2009; Pović et al. 2012) was interpreted as evidence that AGNs heat up the gas in the host-galaxy (Silk \& Rees 1998; Di Matteo et al. 2008) and shut down the star formation rapidly (Bower et al. 2006; Croton et al. 2006; 
Table 9. Values $\rho_{0}$ and $\gamma$ that best fit our co-moving number density calculation (see Eq. (13)) of green valley galaxies at different stellar mass bins and SSP models.

\begin{tabular}{|c|c|c|c|c|c|c|}
\hline & \multicolumn{2}{|c|}{$\mathrm{BC} 03$} & \multicolumn{2}{|c|}{ EMILES+BaSTI } & \multicolumn{2}{|c|}{ EMILES+Padova00 } \\
\hline & $\log _{10} \rho_{0}$ & $\gamma$ & $\log _{10} \rho_{0}$ & $\gamma$ & $\log _{10} \rho_{0}$ & $\gamma$ \\
\hline $10.0 \leq \log _{10} M_{\star}<10.4$ & $-1.42_{-0.53}^{+0.29}$ & $2.64_{-0.77}^{+0.76}$ & $-1.49_{-0.31}^{+0.22}$ & $2.14_{-0.40}^{+0.41}$ & $-1.16_{-0.21}^{+0.15}$ & $2.94_{-0.49}^{+0.52}$ \\
\hline $10.4 \leq \log _{10} M_{\star}<10.8$ & $-2.09_{-0.52}^{+0.34}$ & $1.85_{-0.40}^{+0.40}$ & $-2.13_{-0.39}^{+0.27}$ & $1.61_{-0.27}^{-0.40}$ & $-1.96_{-0.34}^{-0.21}$ & $\begin{array}{r}-0.49 \\
1.79_{-0.29}^{+0.29}\end{array}$ \\
\hline $10.8 \leq \log _{10} M_{\star}<11.2$ & $-1.64_{-0.18}^{+0.15}$ & $2.64_{-0.30}^{+0.31}$ & $-1.46_{-0.10}^{+0.08}$ & $2.72_{-0.20}^{+0.20}$ & $-1.90_{-0.19}^{+0.16}$ & $2.06_{-0.21}^{+0.29}$ \\
\hline $\log _{10} M_{\star} \geq 11.2$ & $-2.23_{-0.64}^{+0.18}$ & $2.35_{-0.58}^{+0.58}$ & $-4.18_{-1.99}^{+0.10}$ & $1.09_{-0.37}^{-0.20}$ & $-2.57_{-0.71}^{+0.19}$ & $1.87_{-0.44}^{-0.21}$ \\
\hline
\end{tabular}

Notes. There are no $\rho_{0}$ and $\gamma$ fitting values for the lowest stellar mass bin, $9.6 \leq \log _{10} M_{\star}<10$, because this subsample is only available at the lowest redshift bin, $0.1 \leq z<0.3$. All the values were obtained at setting $h=1$.

Faber et al. 2007; Schawinski et al. 2007, 2014). In light of our results, AGN feedback should be more efficient than previously expected as a quenching mechanism. Although the detailed study of the green valley galaxies and its evolution across cosmic time is beyond the scope of this work, we provide the co-moving number density $\left(\rho_{\mathrm{N}}\right)$ of this kind of galaxy up to $z \sim 1$ assuming a power-law function of the form

$\rho_{\mathrm{N}}=\rho_{0}(1+z)^{\gamma}$

For this work and by the MCDE, we define the green valley as the loci between the limiting values of quiescent galaxies (see Eq. (3) and Table 3) and a bluer intrinsic colour of $\Delta\left(m_{\mathrm{F} 365}-m_{\mathrm{F} 551}\right)_{\text {int }} \sim 0.2$ with respect to these relations. It should be noted that this definition strictly involves galaxies transiting from the main to the quiescent sequence, but in a more general case green valley galaxies can be treated as third and overlapping galaxy group between quiescent and star-forming galaxies (see e.g. Ilbert et al. 2010). In Table 9 we list the values of $\rho_{0}$ and $\gamma$ that best fit Eq. (13) at different mass bins and SSP models (see also Fig. 10). To associate uncertainties with the comoving number densities, we assumed Poisson errors. Independently of the SSP model set used for the analysis, the number density of green valley galaxies decreases at increasing stellar mass. It is also interesting that the number of massive galaxies $\left(\log _{10} M_{\star} \gtrsim 10.8\right)$ in the green valley decreases at lower redshifts. However, we note that our results at $\log _{10} M_{\star} \lesssim 10.8$ only involve a few points $(z \lesssim 0.5)$ and cosmic variance is not included in the error budget.

Previous studies (Bell et al. 2005; Cowie \& Barger 2008; Brammer et al. 2009; Cardamone et al. 2010; Mahoro et al. 2017) also supported that the population of galaxies in the green valley has bluer intrinsic colours and it is largely dominated by DSF galaxies. Based on the results by Cardamone et al. (2010), $\sim 75 \%$ of the galaxies in the green valley have intrinsic blue colours in good agreement with our work $(\sim 65 \%)$. In the same work and after a dust correction of the $U-V$ colour, most of the galaxies hosting an AGN belong to the red sequence, $(U-V)_{\text {int }} \gtrsim 1.5$, or the blue cloud, $(U-V)_{\text {int }} \lesssim 0.8$, with a poor presence of AGNs at intermediate colours, favouring again the idea of either a faster or less frequent quenching mechanism due to AGNs.

\subsection{Lower $U-V$ colour limit of UVJ-like diagrams}

Even though the rest-frame $U V J$ diagram has been extensively used for the selection of quiescent galaxies, the lower limit of colour $U-V$ is still uncertain or fixed arbitrarily. Some authors propose different definitions of the colour limits to minimise

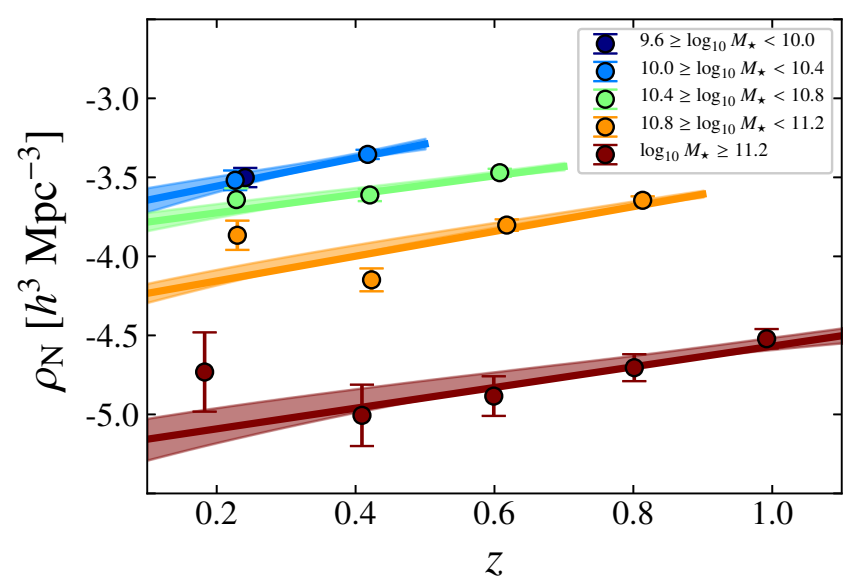

Fig. 10. Evolution of co-moving number density, $\rho_{\mathrm{N}}(z)$, of green valley galaxies with redshift ( $X$-axis) at different stellar mass bins (see inset) using BC03 SSP models. Coloured markers illustrate co-moving number densities at redshift bins and vertical bars are their uncertainties. Solid lines illustrate the best fit to Eq. (13), whereas the shaded regions show the $1 \sigma$ uncertainty of the fit.

the impact of DSF galaxies (see e.g. Moresco et al. 2013). In addition, $U V J$ diagrams are only defined by two colours, where parameters such as stellar mass are not directly accounted for. The detailed analysis of the distribution of stellar population properties in colour-colour diagrams is key to revealing the likely bias that the different colour selections can introduce.

Our results in Sect. 6 point out that low-mass quiescent galaxies lie at the lower limits of the box classically defined for selecting the quiescent population. Therefore, this kind of colour-colour diagram should be built accordingly with the specific features of each photometric survey. Taking advantage of the MCDE (see Fig. 11) we can state that the less massive quiescent galaxies can present $U-V$ colours as blue as the ones of massive star-forming galaxies. These criteria change with redshift because the intrinsic colours of galaxies also vary with cosmic time (see Eq. (3) and Table 3). This is clearly illustrated in Fig. 11 at $0.1 \leq z<0.3$, where a bluer colour limit of $\left(m_{\mathrm{F} 365}-m_{\mathrm{F} 551}<1.5\right)_{\text {int }}$ is necessary to not bias the sample in the low-mass regime. However, this limit must be set in conjunction with the stellar mass to avoid the inclusion of massive star-forming galaxies in the quiescent sample.

In this work, we obtain that the quiescent sample from ALHAMBRA (complete in magnitude down to $m_{\mathrm{F} 814}=23$ ) defined via the dust-corrected $U V J$ colour-colour diagram and the MCDE is equivalent in the stellar mass range for which the quiescent sample is complete in stellar mass (see details in 


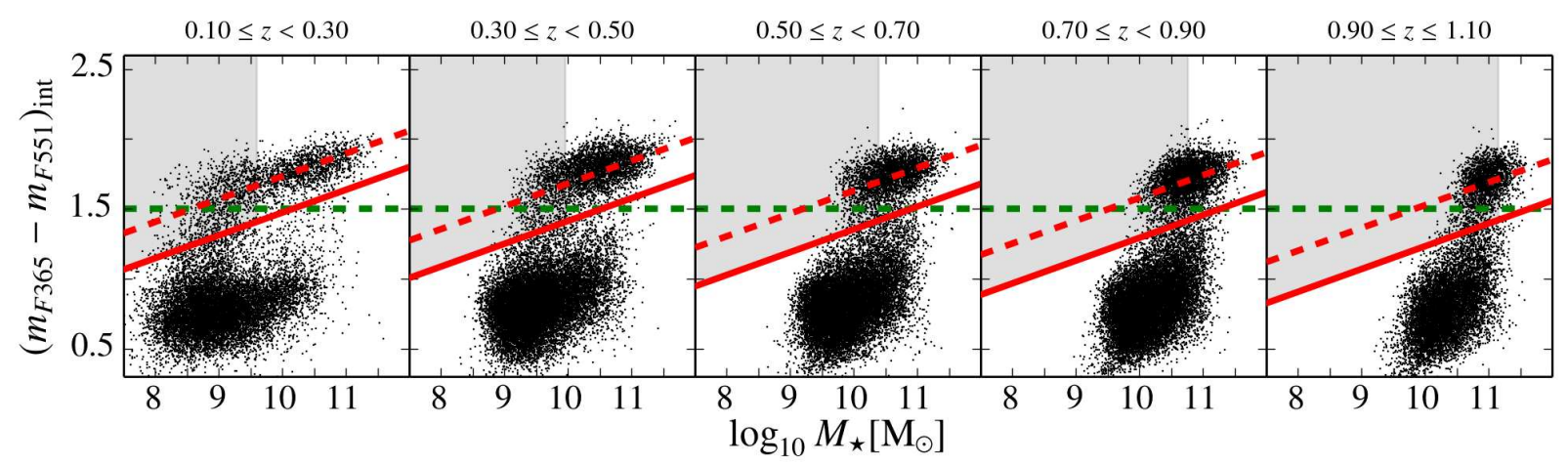

Fig. 11. Galaxies from the ALHAMBRA survey (black dots) in the rest-frame stellar mass-colour diagram corrected for extinction (MCDE) at different redshift bins. The shaded region illustrates the stellar mass range in which our sample of quiescent galaxies is not complete in stellar mass (see Sect. 4.5) at each redshift bin. The dashed green line shows the limiting value $\left(m_{\mathrm{F} 365}-m_{\mathrm{F} 551}\right)_{\text {int }}=1.5$ used to select quiescent galaxies in Sect. 4.1. The dashed red line exhibits the main sequence of quiescent galaxies in the MCDE, see Eq. (3) and Table 3, and the solid line is the limiting colour value used to select quiescent galaxies in this diagram, see Eq. (3) and Table 3. The stellar population predictions were obtained through BC03 SSP models.

Fig. 11). For stellar masses below completeness, the only criteria $\left(m_{\mathrm{F} 365}-m_{\mathrm{F} 551}\right)_{\text {int }}>1.5$ is not enough to select quiescent galaxies. Although dust corrections exhibit a clear bimodality (see also Fig. 1), at $\left(m_{\mathrm{F} 365}-m_{\mathrm{F} 551}\right)_{\text {int }} \sim 1.5$ there is not a welldefined border limiting the two populations in the $U V J$ diagrams. This is because these galaxies present similar colours, even after correcting colours for dust-reddening, and the key to split both populations is the inclusion of stellar mass. This would favour the use of the MCDE with respect to the dust corrected $U V J$ diagrams. Again, we note that in our case the two methods are equivalent to building a quiescent sample complete in stellar mass and luminosity (down to $m_{\mathrm{F} 814}=23$, see Fig. 11).

For deeper surveys, $I>23 \mathrm{mag}$, the selection bias can be even larger if these issues are not taken into account. Less massive quiescent galaxies will be detected, and therefore the $U-V$ lower colour limit should be bluer to not bias them. On the other hand, deeper surveys allow us to explore wider redshift ranges and the use of a unique definition like $\left(m_{\mathrm{F} 365}-m_{\mathrm{F} 551}\right)_{\mathrm{int}}>1.5$ is not strictly adequate because galaxy colours evolve with cosmic time. In a general case and taking the explored dust-corrected diagrams in this work into account, a pure non-biased selection of quiescent galaxies via colour diagrams should include dust corrections of the involved colours and galaxy stellar masses. This discussion can be extended to the classical $U V J$ diagrams in which the colour correction for extinction is not included. Otherwise, samples of red $U V J$ galaxies at low-mass regimes $\left(\log _{10} M_{\star} \sim 9.4\right)$ may include up to $40 \%$ of DSF galaxies. However, the expected contamination for massive quiescent galaxies selected via the non-dust corrected $U V J$ diagram is less affected by this fact (a $2-8 \%$ fraction at $\log _{10} M_{\star}>11$ ), especially at lower redshifts.

\subsection{Dusty star-forming galaxies and flux emission at $24 \mu \mathrm{m}$}

The dust emission at $24 \mu \mathrm{m}$ is an additional SFR tracer (see e.g. Kennicutt 1998) that can be used to confirm the results provided by MUFFIT about DSF galaxies (see Sect. 5). It should be noted that the SFR obtained from the $24 \mu \mathrm{m}$ flux is independent of those provided by MUFFIT using ALHAMBRA data.

The ALHAMBRA field number four (hereafter ALHAMBRA-4) partly overlaps with the S-COSMOS field (Sanders et al. 2007), which allowed us to explore the SFRs of both DSF and quiescent galaxies in a shared subsample. In brief, the S-COSMOS is a deep infrared survey acquired with the Spitzer Space Telescope at the COSMOS field making use of the the Infrared Array Camera (IRAC) and the Multiband Imaging Photometer (MIPS) cameras. We adopted the S-COSMOS MIPS $24 \mu \mathrm{m}$ Photometry Catalog October $2008^{14}$ as a reference data set. This $24 \mu \mathrm{m}$ catalogue is $\sim 90 \%(\sim 75 \%)$ complete above $S_{24 \mu \mathrm{m}} \sim 80 \mu \mathrm{Jy}\left(S_{24 \mu \mathrm{m}} \sim 60 \mu \mathrm{Jy}\right)$, where the full width at half maximum of the MIPS $24 \mu \mathrm{m}$ PSF is $\sim 6^{\prime \prime}$ (Le Floc'h et al. 2009).

To convert the observed flux at $24 \mu \mathrm{m}$ to rest-frame luminosities $\left(L_{24 \mu \mathrm{m}}^{z=0}\right)$, we adopted the interstellar medium (ISM) model proposed by Dale \& Helou (2002) and the photo- $z$ constraints provided by MUFFIT. Briefly, Dale \& Helou (2002) built a SED model of the ISM from combinations of different dust masses assuming a power-law distribution, where the relative contribution of each component in the model is parametrised by a parameter $\alpha$ (see Eq. (1) and Table 2 in Dale \& Helou 2002). Finally, we converted this rest-frame flux to SFRs through the relation provided by Calzetti et al. (2007):

$\mathrm{SFR}_{24 \mu \mathrm{m}}=1.31 \times 10^{-38} \times L_{24 \mu \mathrm{m}}^{z=0} \quad 0.885$

where $\mathrm{SFR}_{24 \mu \mathrm{m}}$ is quoted in $M_{\odot} \mathrm{yr}^{-1}$ units.

After cross-correlating the ALHAMBRA and $24 \mu \mathrm{m}$ MIPS catalogues, we found a total of 1261 common galaxies at $0.2 \leq$ $z \leq 1.2$, where 58 galaxies were labelled as DSF galaxies during our SED fitting analysis. It should be noted that the low number of shared DSF galaxies is mainly due to the reduced overlapping area between the two surveys $\left(\sim 0.25 \mathrm{deg}^{2}\right)$. As a result, $90 \pm 10 \%$ of them (51 DSF galaxies) exhibit $\mathrm{SFR}_{24 \mu \mathrm{m}}$ values higher than the lower limit established by Eq. (10), that is, in the range of star-forming galaxies and reinforcing that these galaxies are reddened by dust. Nevertheless, we find that the subsample of DSF galaxies detected by MIPS is biased to the brightest galaxies, whereas the fainter DSF galaxies may remain under the MIPS detection limit. It is also worth mentioning that discrepancies between the ALHAMBRA and MIPS PSFs $\left(\sim 1^{\prime \prime}\right.$ and $6^{\prime \prime}$, respectively) may be an extra factor to consider for a fair comparison. This may introduce a detection bias because some DSF galaxies would not be deblended and subsequently included in the MIPS catalogue owing to a lower resolution. In this regard, some of the DSF galaxies may show an enhanced $24 \mu \mathrm{m}$ flux due to nearby projected sources. Interestingly, $91 \%$ of the quiescent galaxies in ALHAMBRA-4 were not detected by MIPS. However, this result might be biased owing to the reasons discussed above.

${ }^{14}$ http://irsa.ipac.caltech.edu/data/Cosmos/ 


\subsection{Consistency of the results using alternative attenuation laws}

As our definition of the quiescent sample is based on colours corrected for extinction, the choice of an extinction law may have an impact on our results. To explore whether the choice of another extinction law may vary the main results of this research, we repeated our SED fitting analysis using MUFFIT and the attenuation law of Calzetti (2001) instead of the Fitzpatrick (1999) law.

After running MUFFIT with the attenuation law of Calzetti (2001), we checked the number of quiescent galaxies that we would obtain using the same criteria as presented in Sects. 4.1 and 4.2. As a result, we find that the number of quiescent galaxies differs by around 3\%. These discrepancies in number are slightly bigger for DSF galaxies. The number of DSF galaxies is $15 \%$ larger when using the Calzetti (2001) than the Fitzpatrick (1999) extinction law. Therefore, our results using the Calzetti (2001) law point out that the level of contamination of the quiescent sample owing to the presence of DSF galaxies in $U V J$ diagrams non-corrected for extinction is around $25 \%$, in agreement with the results presented in this work using the Fitzpatrick (1999) extinction law. If we explore the amount of contamination of DSF galaxies in the most and least massive quiescent galaxies of the sample, we obtain again that less massive samples of quiescent galaxies are more affected by this contamination. In particular, quiescent galaxies with stellar masses around $\log _{10} M_{\star}=9.6$ dex present a $30 \%$ fraction of DSF galaxies when they are selected through a classical $U V J$ diagram, whereas for $\log _{10} M_{\star}>11.2$ dex this number ranges from $5 \%$ to $15 \%$ at $0.1<z<0.3$ and $0.9<z<1.1$, respectively.

If we explore the presence of DSF galaxies in the green valley using the Calzetti (2001) attenuation law, we obtain the same result as using the Fitzpatrick (1999) extinction law. A relevant number of the galaxies that populate the green valley are actually obscured star-forming galaxies. Assuming the same limits for the green valley as in Sects. 4.1 and 4.2 (i.e. $0.1 \mathrm{mag}$ above and below the limiting values expressed by Eqs. (1) and (3) for the $U V J$ and stellar mass-colour diagrams, respectively), the number of DSF galaxies in the green valley is in the range of 25-60\%.

Finally, we confront one-by-one the stellar population properties obtained using the Calzetti (2001) and Fitzpatrick (1999) extinction laws for the subsample of quiescent galaxies complete in stellar mass. Our analysis reveals a good agreement between the two predictions and without the presence of systematics. The main consequence of using different attenuation assumptions during the stellar population analysis of quiescent galaxies via SED fitting is the scatter. From this test, we quantify that the scatter is typically below 0.05 dex for all the parameters explored in this work, except for metallicity that amounts to $0.15 \mathrm{dex}$, but without systematics. This result is not surprising because, as revealed in this study, the values of extinction in quiescent galaxies are typically low (average value of $A_{V} \sim 0.3$ ). We find that the use of different synthesis models, such as BC03 and EMILES, is a much larger source of systematics than the use of different extinction or attenuation laws (see also Díaz-García et al. 2019a).

\section{Summary and conclusions}

Using the data set provided by the ALHAMBRA multi-filter photometric survey and our optimised SED fitting tool MUFFIT (extensively detailed in Díaz-García et al. 2015) with the BC03 and EMILES SSP models, we explored the stellar content of quiescent galaxies since $z=1.1$, corresponding to the last 8 Gyr ( $60 \%$ of the age of the Universe), and their distributions in the dust-corrected stellar mass-colour and the $U V J$ colourcolour diagrams.

The selection of the quiescent sample was carefully performed to minimise as much as possible the number of contaminants, mainly faint cool stars and dusty star-forming galaxies. In brief, we improved the colour-colour $U V J$ diagram taking the extinctions provided by MUFFIT into account to generate a dust-corrected $U V J$ diagram, which minimises the contamination by dusty star-forming galaxies. We also explored the use of stellar mass-colour diagrams corrected for extinction (MCDE), an alternative diagram for the selection of quiescent galaxies, and compared the results with to those from the $U V J$ diagram. Moreover, we carried out a one-by-one visual inspection to remove those sources whose photometry might be compromised (e.g. with bad CCD regions or bad photometric apertures) and spurious detections. Based on SED fitting techniques, we removed cool stars from the ALHAMBRA catalogue with magnitudes ranging $22.5 \leq m_{\mathrm{F} 814 \mathrm{~W}} \leq 23$. After comparison with the morphological classification of COSMOS for a common sample of sources, the contamination due to cool stars was reduced from $24 \%$ to $4 \%$. Finally, we estimated the stellar mass completeness of our sample of quiescent galaxies in ALHAMBRA by applying a novel method for defining an analytic function for the stellar mass completeness that yields a final sample of $\sim 8500$ quiescent galaxies at $0.1 \leq z \leq 1.1$ with a photo- $z$ accuracy of $\sigma_{\mathrm{NMAD}}=0.006$.

We also developed a reliable methodology for taking advantage of our SED fitting results (based on a mixture of two SSPs) and provide SFR and sSFR values taking the luminosity at $2800 \AA$ as SFR tracer. The exploration of the distribution of SFR and SSFR values in the stellar mass plane reveals a bimodality, where the main sequence galaxies (star-forming galaxies) populate the upper SFR and sSFR values. In addition, the more massive quiescent galaxies exhibit higher SFRs than their lower mass counterparts. However, the most efficient process of star formation in the quiescent population resides in the low-mass systems, whereas the massive ones present the lowest sSFRs of the quiescent population.

From the dust-corrected $U V J$-diagram, we find a number of striking results:

- A relevant number of the galaxies that reside in the green valley are actually obscured star-forming galaxies $(\sim 30-65 \%)$. These reveal an intrinsic colour $\left(m_{\mathrm{F} 365}-m_{\mathrm{F} 551}\right)_{\text {int }}$ characteristic of the star-forming population, although their great dust content significantly reddens their colours. This also implies that the green valley is less populated than expected and the transition of galaxies from the blue cloud to the red sequence, and hence the related mechanisms for quenching, seems to be much more efficient and faster than previously considered. As this result may be very important to derive the quenching timescales of galaxies in future papers, we also provide the co-moving number densities of green valley galaxies after accounting for dust effects.

- Down to $m_{\mathrm{F} 814 \mathrm{~W}}=23$, the histogram of the $\left(m_{\mathrm{F} 365}-m_{\mathrm{F} 551}\right)_{\text {int }}$ colour exhibits a local minimum at $\sim 1.5$ that can be imposed as the bluest colour limit to fairly select the quiescent sample, which remains roughly constant since $z \leq 1.1$.

- Red galaxies that belong to the star-forming sample after the dust correction (intrinsic $m_{\mathrm{F} 365}-m_{\mathrm{F} 551}<1.5$ ) are typically concentrated close to the edges of $U V J$ diagrams (e.g. Williams et al. 2009; Moresco et al. 2013), supporting the reliability of the extinction values provided by MUFFIT. 
- Quiescent galaxies selected through a classical $U V J$ diagram (not corrected for dust effects) are typically contaminated by a $\sim 20 \%$ fraction of dusty star-forming galaxies. Our results clearly establish that this contamination is less severe for massive galaxies $\left(\log _{10} M_{\star} \geq 11,2-8 \%\right.$ from $z \sim 0.1$ to $z \sim 1.1)$ than for the less massive ones $(40 \%$, for $9.2 \leq \log _{10} M_{\star} \leq 9.6$ at $0.1 \leq z \leq 0.3$ ).

The analysis of the distribution of stellar population parameters of quiescent galaxies on a dust-corrected $U V J$-diagram and MCDE reveals that there is a close correlation between the position of each galaxy in these diagrams and its age, metallicity, extinction, and stellar mass. We conclude the following:

- The more massive quiescent galaxies lie on the redder parts of the $U V J$ diagram at greater cosmic times. At higher redshifts, $z \sim 1$, massive quiescent galaxies present a wider spread of $\left(m_{\mathrm{F} 365}-m_{\mathrm{F} 551}\right)_{\text {int }}$ colours, $1.5 \leq\left(m_{\mathrm{F} 365}-m_{\mathrm{F} 551}\right)_{\text {int }} \leq$ 2.0 , than at $z \sim 0.2$, where they lie on the upper and redder parts of the $U V J$ diagram, $\left(m_{\mathrm{F} 365}-m_{\mathrm{F} 551}\right)_{\text {int }} \sim 2.0$.

- The whole quiescent sample shows an expected low dust content ( $96 \%$ of the galaxies present $A_{V} \leq 0.6$ ), where quiescent galaxies with bluer intrinsic colours, $\left(m_{\mathrm{F} 365}-m_{\mathrm{F} 551}\right)_{\text {int }} \lesssim$ 1.7 and $\left(m_{\mathrm{F} 551}-J\right)_{\text {int }} \lesssim 1.2$, are also the galaxies with higher extinction values $\left(A_{V} \gtrsim 0.4\right)$. The dust-corrected colours $\left(m_{\mathrm{F} 365}-m_{\mathrm{F} 551}\right)_{\text {int }}$ and $\left(m_{\mathrm{F} 551}-J\right)_{\text {int }}$ exhibit a remarkable anticorrelation with extinction with Spearman correlation coefficients of $\rho_{U V} \sim-0.92$ and $\rho_{V J} \sim-0.75$, respectively.

- We find a correlation between the colour $\left(m_{\mathrm{F} 365}-m_{\mathrm{F} 551}\right)_{\text {int }}$ and the age of quiescent galaxies with a value of $\rho_{U V} \sim 0.45$. The older ages present redder $\left(m_{\mathrm{F} 365}-m_{\mathrm{F} 551}\right)_{\text {int }}$ colours, populating the upper parts of the $U V J$ diagram, $\left(m_{\mathrm{F} 365}-\right.$ $\left.m_{\mathrm{F} 551}\right)_{\text {int }}>1.8$. On the other hand, the younger quiescent galaxies present the bluer colours of the diagram. Although there is a correlation with the $\left(m_{\mathrm{F} 365}-m_{\mathrm{F} 551}\right)$ int colour, age is also slightly dependent on the $\left(m_{\mathrm{F} 551}-J\right)_{\text {int }}$ colour $\left(\rho_{V J} \sim 0.15\right)$. The older quiescent galaxies lie on the highmass end of the MCDE and these are the reddest ones (both $\left(m_{\mathrm{F} 365}-m_{\mathrm{F} 551}\right)_{\text {int }}$ and $\left.m_{\mathrm{F} 365}-m_{\mathrm{F} 551}\right)$. The less massive quiescent galaxies are younger and bluer, in good agreement with the downsizing scenario.

- The metallicity distribution in the $U V J$ diagram is tightly correlated with the colour $\left(m_{\mathrm{F} 551}-J\right)_{\text {int }}$ and less remarkably with $\left(m_{\mathrm{F} 365}-m_{\mathrm{F} 551}\right)_{\text {int }}$, with correlation coefficients $\rho_{V J} \sim 0.85$ and $\rho_{U V} \sim 0.45$, respectively. The most metalrich quiescent galaxies $\left([\mathrm{M} / \mathrm{H}]_{\mathrm{M}}>0.1 \mathrm{dex}\right)$ also present the reddest colours $\left(m_{\mathrm{F} 551}-J\right)_{\mathrm{int}}>1.1$. The more massive the quiescent galaxy, the more metal-rich it is, as revealed by the MCDE. Metallicity values lie on very well-defined positions in the $U V J$ and the MCDE diagrams.

- For quiescent galaxies, very low sSFR $\left(\log _{10} \mathrm{SSFR}_{2800 \AA} \sim\right.$ $\left.-11 \mathrm{yr}^{-1}\right)$ lie on the upper parts of rest-frame and dust corrected $U V J$ diagrams $\left(\left(m_{\mathrm{F} 365}-m_{\mathrm{F} 551}\right)_{\text {int }} \gtrsim 1.8\right)$, while values of $\log _{10} \mathrm{SSFR}_{2800 \AA} \sim-10.5$ are at $\left(m_{\mathrm{F} 365}-m_{\mathrm{F} 551}\right)_{\text {int }} \lesssim 1.6$. An anticorrelation between sSFR and the intrinsic colour $\left(m_{\mathrm{F} 365}-m_{\mathrm{F} 551}\right)_{\mathrm{int}}$ is found close to -1 , which may be used as an sSFR tracer at least for quiescent galaxies. Actually, this tracer should be treated carefully as the sSFRs are computed via a SFR tracer, which is linked to the $2800 \AA$ luminosity, and the continuum yields a non-null sSFR value even when a star formation process is not present.

From both stellar mass-colour and UVJ colour-colour diagrams, we conclude that dust corrections play an important role in understanding how quiescent galaxies distribute inside these diagrams as a function of their parameters: mass, age, metallicity, extinction, and sSFR. Without dust corrections, quiescent galaxies with high dust content are in the upper parts of the $U V J$ diagram and CMD as predicted by extinction laws. The correlation between age and colour $\left(m_{\mathrm{F} 365}-m_{\mathrm{F} 551}\right)$ is weaker than with $\left(m_{\mathrm{F} 365}-m_{\mathrm{F} 551}\right)_{\text {int }}$, whereas metallicity still correlates with $\left(m_{\mathrm{F} 551}-J\right)$, but also with $\left(m_{\mathrm{F} 365}-m_{\mathrm{F} 551}\right)$.

After exploring the MCDE and the rest-frame $U V J$ colourcolour diagram, we note that to perform a pure non-biased selection of quiescent galaxies from these diagrams, we should include dust corrections of the involved colours and also the galaxy stellar mass. This will allow us to develop future and more reliable stellar population studies without biasing the quiescent galaxy sample, and to reveal the evolution of related properties with cosmic time.

We also quantify the distributions of stellar population properties (extinction, age, metallicity, and sSFR) within the restframe $U V J$ and MCDE diagrams corrected for extinction, which may be very useful for future works to estimate stellar population parameters by the positions of quiescent galaxies within these diagrams.

As this research involves dust-corrected colours, the choice of an extinction law during the SED fitting analysis may have an impact on the sample definition and results obtained in this work. However, we find that the main results in this work are consistent when we use the extinction laws of Fitzpatrick (1999) and Calzetti (2001) separately.

Acknowledgements. The authors are grateful to the referee for their fruitful comments, which contributed to improving the present research. This work has been supported by the Programa Nacional de Astronomía y Astrofísica of the Spanish Ministry of Economy and Competitiveness (MINECO, grants AYA2012-30789 and AYA2015-66211-C2-1-P), by the Ministry of Science and Technology of Taiwan (grant MOST 106-2628-M-001-003-MY3), by the Academia Sinica (grant AS-IA-107-M01), and by the Government of Aragón (Research Group E103). L. A. D. G. acknowledges support from the Caja Rural de Teruel. L. A. D. G. also thanks I. F. for offering the opportunity to develop part of this research at the Mullard Space Science Laboratory (MSSL). We also acknowledge support from the Spanish Ministry for Economy and Competitiveness and FEDER funds through grants AYA2010-15081, AYA2010-15169, AYA2010-22111-C03-01, AYA2010-22111-C03-02, AYA2011-29517-C03-01, AYA2012-39620, AYA2013-40611-P, AYA2013-42227-P, AYA2013-43188-P, AYA2013-48623-C2-1, AYA2013-48623-C2-2, ESP2013-48274, AYA201457490-P, AYA2014-58861-C3-1, AYA2016-76682-C3-1-P, AYA2016-77846-P, AYA2016-81065-C2-1, AYA2016-81065-C2-2; Generalitat Valenciana projects Prometeo 2009/064 and PROMETEOII/2014/060; Junta de Andalucía grants TIC114, JA2828, P10-FQM-6444; and Generalitat de Catalunya project SGR1398. Throughout this research, we made use of the Matplotlib package (Hunter 2007), a 2D graphics package used for Python that is designed for interactive scripting and quality image generation. This paper is dedicated to Marian León Canalejo for being there when L. A. D. G. needed her most and for her patience and continuous encouragement he was finishing his $\mathrm{PhD}$.

\section{References}

Aparicio Villegas, T., Alfaro, E. J., Cabrera-Caño, J., et al. 2010, AJ, 139, 1242 Arnouts, S., Le Floc'h, E., Chevallard, J., et al. 2013, A\&A, 558, A67 Arzner, K., Güdel, M., Briggs, K., et al. 2007, A\&A, 468, 501 Baldry, I. K., Glazebrook, K., Brinkmann, J., et al. 2004, ApJ, 600, 681 Baldry, I. K., Balogh, M. L., Bower, R. G., et al. 2006, MNRAS, 373, 469 Balogh, M. L., Morris, S. L., Yee, H. K. C., Carlberg, R. G., \& Ellingson, E. 1999, ApJ, 527, 54

Bell, E. F., Wolf, C., Meisenheimer, K., et al. 2004, ApJ, 608, 752

Bell, E. F., Papovich, C., Wolf, C., et al. 2005, ApJ, 625, 23

Belli, S., Newman, A. B., \& Ellis, R. S. 2015, ApJ, 799, 206

Benítez, N. 2000, ApJ, 536, 571

Bower, R. G., Lucey, J. R., \& Ellis, R. S. 1992, MNRAS, 254, 601

Bower, R. G., Benson, A. J., Malbon, R., et al. 2006, MNRAS, 370, 645

Brammer, G. B., van Dokkum, P. G., \& Coppi, P. 2008, ApJ, 686, 1503

Brammer, G. B., Whitaker, K. E., van Dokkum, P. G., et al. 2009, ApJ, 706, L173

Brown, M. J. I., Dey, A., Jannuzi, B. T., et al. 2007, ApJ, 654, 858

Bruzual, A. G. 1983, ApJ, 273, 105

Bruzual, G., \& Charlot, S. 2003, MNRAS, 344, 1000 
Bundy, K., Georgakakis, A., Nandra, K., et al. 2008, ApJ, 681, 931 Calzetti, D. 2001, PASP, 113, 1449

Calzetti, D., Kennicutt, R. C., Engelbracht, C. W., et al. 2007, ApJ, 666, 870

Capak, P., Aussel, H., Ajiki, M., et al. 2007, ApJS, 172, 99

Cappellari, M., McDermid, R. M., Alatalo, K., et al. 2013, MNRAS, 432, 1862

Cardamone, C. N., Urry, C. M., Schawinski, K., et al. 2010, ApJ, 721, L38

Chabrier, G. 2003, PASP, 115, 763

Choi, J., Conroy, C., Moustakas, J., et al. 2014, ApJ, 792, 95

Cid Fernandes, R., Mateus, A., Sodré, L., Stasińska, G., \& Gomes, J. M. 2005 , MNRAS, 358, 363

Cleveland, W. S. 1979, J. Am. Stat. Assoc., 74, 829

Cleveland, W., \& Devlin, S. 1988, J. Am. Stat. Assoc., 83, 596

Coe, D., Benítez, N., Sánchez, S. F., et al. 2006, AJ, 132, 926

Coelho, P., Barbuy, B., Meléndez, J., Schiavon, R. P., \& Castilho, B. V. 2005, A\&A, 443, 735

Conroy, C. 2013, ARA\&A, 51, 393

Cooper, M. C., Aird, J. A., Coil, A. L., et al. 2011, ApJS, 193, 14

Cowie, L. L., \& Barger, A. J. 2008, ApJ, 686, 72

Cowie, L. L., Songaila, A., Hu, E. M., \& Cohen, J. G. 1996, AJ, 112, 839

Cristóbal-Hornillos, D., Aguerri, J. A. L., Moles, M., et al. 2009, ApJ, 696, 1554

Croton, D. J., Springel, V., White, S. D. M., et al. 2006, MNRAS, 365, 11

Daddi, E., Cimatti, A., Renzini, A., et al. 2004, ApJ, 617, 746

Daddi, E., Dickinson, M., Morrison, G., et al. 2007, ApJ, 670, 156

Dale, D. A., \& Helou, G. 2002, ApJ, 576, 159

Davis, M., Guhathakurta, P., Konidaris, N. P., et al. 2007, ApJ, 660, L1

Di Matteo, T., Colberg, J., Springel, V., Hernquist, L., \& Sijacki, D. 2008, ApJ 676,33

Díaz-García, L. A., Cenarro, A. J., López-Sanjuan, C., et al. 2015, A\&A, 582 A14

Díaz-García, L. A., Cenarro, A. J., López-Sanjuan, C., et al. 2019a, A\&A, 631, A157

Díaz-García, L. A., Cenarro, A. J., López-Sanjuan, C., et al. 2019b, A\&A, 631, A158

Domínguez Sánchez, H., Pozzi, F., Gruppioni, C., et al. 2011, MNRAS, 417, 900

Domínguez Sánchez, H., Pérez-González, P. G., Esquej, P., et al. 2016, MNRAS 457,3743

Elbaz, D., Daddi, E., Le Borgne, D., et al. 2007, A\&A, 468, 33

Faber, S. M., Willmer, C. N. A., Wolf, C., et al. 2007, ApJ, 665, 265

Fang, J. J., Faber, S. M., Koo, D. C., et al. 2018, ApJ, 858, 100

Fitzpatrick, E. L. 1999, PASP, 111, 63

Fontana, A., Pozzetti, L., Donnarumma, I., et al. 2004, A\&A, 424, 23

Foreman-Mackey, D., Hogg, D. W., Lang, D., \& Goodman, J. 2013, PASP, 125 306

Fritz, A., Scodeggio, M., Ilbert, O., et al. 2014, A\&A, 563, A92

Fumagalli, M., Labbé, I., Patel, S. G., et al. 2014, ApJ, 796, 35

Gallazzi, A., Charlot, S., Brinchmann, J., White, S. D. M., \& Tremonti, C. A 2005, MNRAS, 362, 41

Gallazzi, A., Charlot, S., Brinchmann, J., \& White, S. D. M. 2006, MNRAS, 370,1106

Georgakakis, A., Nandra, K., Yan, R., et al. 2008, MNRAS, 385, 2049

Girardi, L., Bressan, A., Bertelli, G., \& Chiosi, C. 2000, A\&AS, 141, 371

González Delgado, R. M., Cid Fernandes, R., García-Benito, R., et al. 2014a, ApJ, 791, L16

González Delgado, R. M., Pérez, E., Cid Fernandes, R., et al. 2014b, A\&A, 562, A47

Hickox, R. C., Jones, C., Forman, W. R., et al. 2009, ApJ, 696, 891

Hunter, J. D. 2007, Comput. Sci. Eng., 9, 90

Ilbert, O., Salvato, M., Le Floc'h, E., et al. 2010, ApJ, 709, 644

Ilbert, O., McCracken, H. J., Le Fèvre, O., et al. 2013, A\&A, 556, A55

Jimenez, R., Bernardi, M., Haiman, Z., Panter, B., \& Heavens, A. F. 2007, ApJ 669,947

Kauffmann, G., Heckman, T. M., White, S. D. M., et al. 2003, MNRAS, 341, 54

Kennicutt, Jr., R. C. 1998, ARA\&A, 36, 189

Koleva, M., \& Vazdekis, A. 2012, A\&A, 538, A143

Kroupa, P. 2001, MNRAS, 322, 231

Le Floc'h, E., Aussel, H., Ilbert, O., et al. 2009, ApJ, 703, 222

Leauthaud, A., Massey, R., Kneib, J.-P., et al. 2007, ApJS, 172, 219

Lilly, S. J., Le Brun, V., Maier, C., et al. 2009, ApJS, 184, 218

López-Sanjuan, C., García-Dabó, C. E., \& Balcells, M. 2008, PASP, 120, 571

López-Sanjuan, C., Cenarro, A. J., Hernández-Monteagudo, C., et al. 2014, A\&A, 564, A127

López-Sanjuan, C., Cenarro, A. J., Hernández-Monteagudo, C., et al. 2015 A\&A, 582, A16

Madau, P., Pozzetti, L., \& Dickinson, M. 1998, ApJ, 498, 106
Mahoro, A., Pović, M., \& Nkundabakura, P. 2017, MNRAS, 471, 3226 Makarov, D., Makarova, L., Rizzi, L., et al. 2006, AJ, 132, 2729

Martis, N. S., Marchesini, D., Brammer, G. B., et al. 2016, ApJ, 827, L25 McDermid, R. M., Alatalo, K., Blitz, L., et al. 2015, MNRAS, 448, 3484

Moles, M., Benítez, N., Aguerri, J. A. L., et al. 2008, AJ, 136, 1325

Molino, A., Benítez, N., Moles, M., et al. 2014, MNRAS, 441, 2891

Moresco, M., Pozzetti, L., Cimatti, A., et al. 2013, A\&A, 558, A61

Moustakas, J., Coil, A. L., Aird, J., et al. 2013, ApJ, 767, 50

Nandra, K., Georgakakis, A., Willmer, C. N. A., et al. 2007, ApJ, 660, L11

Naylor, T., \& Jeffries, R. D. 2006, MNRAS, 373, 1251

Noeske, K. G., Weiner, B. J., Faber, S. M., et al. 2007, ApJ, 660, L43

Oke, J. B., \& Gunn, J. E. 1983, ApJ, 266, 713

Pacifici, C., Kassin, S. A., Weiner, B. J., et al. 2016, ApJ, 832, 79

Panter, B., Jimenez, R., Heavens, A. F., \& Charlot, S. 2008, MNRAS, 391, 1117

Peletier, R. F. 2013, XXIII Canary Islands Winter School of Astrophysics. Stellar Populations, 353

Peng, Y.-J., Lilly, S. J., Kovač, K., et al. 2010, ApJ, 721, 193

Peng, Y., Maiolino, R., \& Cochrane, R. 2015, Nature, 521, 192

Pérez-González, P. G., Rieke, G. H., Villar, V., et al. 2008, ApJ, 675, 234

Pietrinferni, A., Cassisi, S., Salaris, M., \& Castelli, F. 2004, ApJ, 612, 168

Pović, M., Huertas-Company, M., Aguerri, J. A. L., et al. 2013, MNRAS, 435, 3444

Pović, M., Sánchez-Portal, M., Pérez García, A. M., et al. 2012, A\&A, 541, A118

Pozzetti, L., Bolzonella, M., Zucca, E., et al. 2010, A\&A, 523, A13

Ricciardelli, E., Vazdekis, A., Cenarro, A. J., \& Falcón-Barroso, J. 2012, MNRAS, 424, 172

Röck, B., Vazdekis, A., Peletier, R. F., Knapen, J. H., \& Falcón-Barroso, J. 2015, MNRAS, 449, 2853

Salpeter, E. E. 1955, ApJ, 121, 161

Sandage, A., Tammann, G. A., \& Yahil, A. 1979, ApJ, 232, 352

Sanders, D. B., Salvato, M., Aussel, H., et al. 2007, ApJS, 172, 86

Schawinski, K., Thomas, D., Sarzi, M., et al. 2007, MNRAS, 382, 1415

Schawinski, K., Virani, S., Simmons, B., et al. 2009, ApJ, 692, L19

Schawinski, K., Urry, C. M., Simmons, B. D., et al. 2014, MNRAS, 440, 889

Schechter, P. 1976, ApJ, 203, 297

Schlegel, D. J., Finkbeiner, D. P., \& Davis, M. 1998, ApJ, 500, 525

Silk, J., \& Rees, M. J. 1998, A\&A, 331, L1

Silverman, J. D., Mainieri, V., Lehmer, B. D., et al. 2008, ApJ, 675, 1025

Trager, S. C., Faber, S. M., Worthey, G., \& González, J. J. 2000, AJ, 120, 165

Tremonti, C. A., Heckman, T. M., Kauffmann, G., et al. 2004, ApJ, 613, 898

Vazdekis, A., Cenarro, A. J., Gorgas, J., Cardiel, N., \& Peletier, R. F. 2003, MNRAS, 340, 1317

Vazdekis, A., Sánchez-Blázquez, P., Falcón-Barroso, J., et al. 2010, MNRAS, 404, 1639

Vazdekis, A., Ricciardelli, E., Cenarro, A. J., et al. 2012, MNRAS, 424, 157

Vazdekis, A., Koleva, M., Ricciardelli, E., Röck, B., \& Falcón-Barroso, J. 2016, MNRAS, 463, 3409

Vergani, D., Scodeggio, M., Pozzetti, L., et al. 2008, A\&A, 487, 89

Whitaker, K. E., van Dokkum, P. G., Brammer, G., et al. 2010, ApJ, 719, 1715

Whitaker, K. E., van Dokkum, P. G., Brammer, G., \& Franx, M. 2012, ApJ, 754 L29

Williams, R. J., Quadri, R. F., Franx, M., van Dokkum, P., \& Labbé, I. 2009, ApJ, 691, 1879

Worthey, G., Faber, S. M., Gonzalez, J. J., \& Burstein, D. 1994, ApJS, 94, 687

Wyder, T. K., Martin, D. C., Schiminovich, D., et al. 2007, ApJS, 173, 293

Yano, M., Kriek, M., van der Wel, A., \& Whitaker, K. E. 2016, ApJ, 817, L21

1 Centro de Estudios de Física del Cosmos de Aragón (CEFCA), Plaza San Juan 1, Floor 2, 44001 Teruel, Spain

2 Academia Sinica Institute of Astronomy \& Astrophysics (ASIAA), 11F of Astronomy-Mathematics Building, AS/NTU, No. 1, Section 4, Roosevelt Road, Taipei 10617, Taiwan

e-mail: ladiaz@asiaa.sinica.edu.tw, ladg84@gmail.com

3 Centro de Estudios de Física del Cosmos de Aragón (CEFCA) Unidad Asociada al CSIC, Plaza San Juan 1, Floor 2, 44001 Teruel, Spain

4 Mullard Space Science Laboratory, University College London, Holmbury St Mary, Dorking, Surrey RH5 6NT, UK

5 IAA-CSIC, Glorieta de la Astronomía s/n, 18008 Granada, Spain

6 Instituto de Astrofísica de Canarias, Vía Láctea s/n, 38200 La Laguna, Tenerife, Spain 
L. A. Díaz-García et al.: Stellar populations of galaxies within diagrams involving colours

7 Instituto de Física de Cantabria (CSIC-UC), 39005 Santander, Spain

${ }^{8}$ Unidad Asociada Observatorio Astronómico (IFCA-UV), 46980 Paterna, Spain

9 Observatório Nacional-MCT, Rua José Cristino, 77. CEP, 20921400 Rio de Janeiro-RJ, Brazil

10 Department of Theoretical Physics, University of the Basque Country UPV/EHU, 48080 Bilbao, Spain

11 IKERBASQUE, Basque Foundation for Science, Bilbao, Spain

12 Departamento de Física Atómica, Molecular y Nuclear, Facultad de Física, Universidad de Sevilla, 41012 Sevilla, Spain

13 Institut de Ciències de l'Espai (IEEC-CSIC), Facultat de Ciències, Campus UAB, 08193 Bellaterra, Spain
14 Departamento de Astrofísica, Facultad de Física, Universidad de La Laguna, 38206 La Laguna, Spain

15 Instituto de Astrofísica, Universidad Católica de Chile, Av. Vicuna Mackenna 4860, 782-0436 Macul, Santiago, Chile

16 Centro de Astro-Ingeniería, Universidad Católica de Chile, Av. Vicuna Mackenna 4860, 782-0436 Macul, Santiago, Chile

17 Observatori Astronòmic, Universitat de València, C/ Catedràtic José Beltrán 2, 46980 Paterna, Spain

18 Departament d'Astronomia i Astrofísica, Universitat de València, 46100 Burjassot, Spain

19 Instituto de Astronomía, Geofísica e Ciéncias Atmosféricas, Universidade de São Paulo, São Paulo, Brazil 


\section{Appendix A: Removal of uncertainty effects from distributions of stellar population properties}

During the last decades, maximum likelihood estimator (MLE) methods have been used for various aims in astronomy (e.g. Naylor \& Jeffries 2006; Makarov et al. 2006; Arzner et al. 2007; López-Sanjuan et al. 2008, 2015). For this work, we performed an MLE to remove uncertainty effects of individual galaxies from distributions of stellar population properties, that is, a deconvolution to recover the intrinsic distributions of stellar population parameters. In Sects. 4.2 and 5, the distributions of $\left(m_{\mathrm{F} 365}-m_{\mathrm{F} 551}\right)_{\text {int }}^{\mathrm{rot}}$ and $\log _{10} \mathrm{SFR}_{2800 \AA}^{\mathrm{rot}}$ values (Eqs. (2) and (11), respectively) presented a challenge to determine the limiting values for quiescent galaxies because these distributions are affected by the redshift-dependent uncertainties of individual galaxies.

Our starting point is the MLE method detailed by LópezSanjuan et al. (2014), which was initially developed to disentangle cosmic variance effects. In López-Sanjuan et al. (2014), the authors assumed that intrinsic distributions are properly described by Gaussian-like probability distributions in the logspace, that is, log-normal distributions in the real space. In addition, for uncertainties in the stellar population parameters of individual galaxies a Gaussian distribution was also assumed. Therefore, uncertainty effects would increase distribution widths with little impact on the distribution median. Under these assumptions, the likelihood to maximise is

$\mathcal{L}=-\frac{1}{2} \sum_{j}\left[\ln \left(p_{\mathrm{e}, j}{ }^{2}+\sigma_{\mathrm{p}}^{\mathrm{int}^{2}}\right)+\frac{\left(\mu_{\mathrm{p}}-p_{j}\right)^{2}}{p_{\mathrm{e}, j}^{2}+\sigma_{\mathrm{p}}^{\mathrm{int}^{2}}}\right]$,

where $p_{j}$ is the stellar population parameter (in this work $\left(m_{\mathrm{F} 365}-m_{\mathrm{F} 551}\right)_{\text {int }}^{\text {rot }}$ or $\left.\log _{10} \mathrm{SFR}_{2800 \AA}^{\mathrm{rot}}\right)$ of the $j$ th galaxy in the distribution, $p_{\mathrm{e}, j}$ is its $1 \sigma$ error, $\mu_{\mathrm{p}}$ the median of the real or intrinsic distribution (without uncertainty effects), and $\sigma_{\mathrm{p}}^{\text {int }}$ its width or intrinsic dispersion (for details, see López-Sanjuan et al. 2014). For the maximisation of Eq. (A.1), we made the most of the emcee $^{15}$ algorithm (Foreman-Mackey et al. 2013). This tool is an affine invariant sampling algorithm for a Markov chain Monte Carlo method (MCMC) that can be easily adapted to any function for maximisation.

Figure A.1 shows the distribution of $\left(m_{\mathrm{F} 365}-m_{\mathrm{F} 551}\right)_{\text {int }}^{\text {rot }}$ values of quiescent galaxies (for further details, see Sect. 4.2). It should be noted that Eq. (A.1) is defined for Gaussian distributions, and $\left(m_{\mathrm{F} 365}-m_{\mathrm{F} 551}\right)_{\text {int }}^{\mathrm{rot}}$ is a log-normal distribution (see Fig. A.1). Therefore, we maximised Eq. (A.1) for the distribution of values in the log-space, that is, $\ln \left[1-\left(m_{\mathrm{F} 365}-m_{\mathrm{F} 551}\right)_{\mathrm{int}}^{\mathrm{rot}}\right]$, where a shift in the distribution is necessary to avoid negative values. As expected, medians almost remain unaltered after the MLE deconvolution, whereas the intrinsic distributions are narrower than the observed values (see Fig. A.1). To illustrate, close to the $3 \sigma$ limit there is an overdensity in the number of galaxies, which may be partly interpreted as the transient population of galaxies from the blue cloud to the red sequence (green valley, see Fig. A.1), but also by uncertainties.

The intrinsic distribution of $\log _{10} \mathrm{SFR}_{2800 \AA}^{\mathrm{rot}}$ can be fitted by Gaussian functions at any redshift bin properly (see Fig. A.2). Once again, the most remarkable impact of uncertainties is the widening of the distributions. After removing uncertainties and parametrising the distribution, it is straightforward to set the limiting sSFR values of quiescent galaxies. As mentioned above, we set this limit at a $3 \sigma$ level (see Fig. A.2).

15 http://dan.iel.fm/emcee

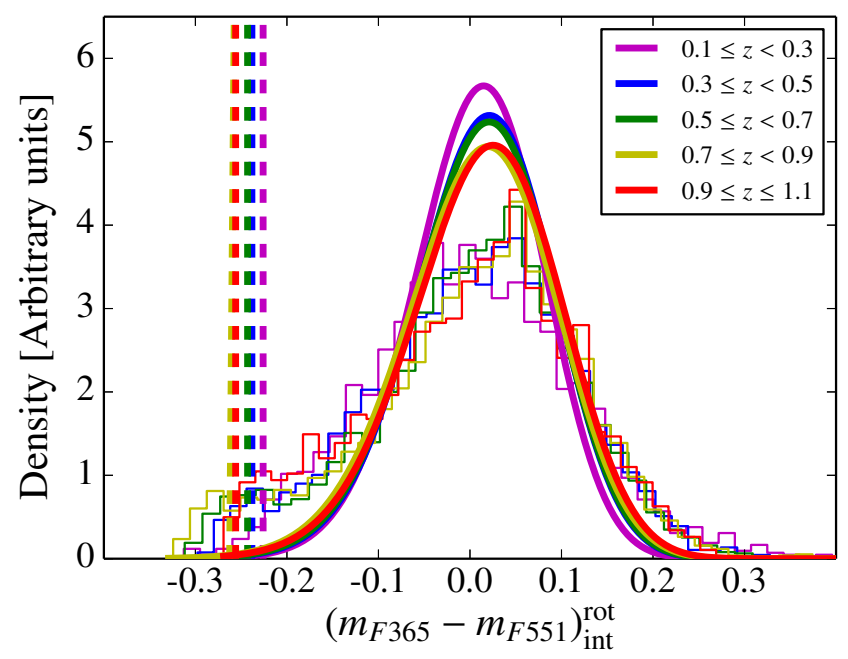

Fig. A.1. Histogram of the $\left(m_{\mathrm{F} 365}-m_{\mathrm{F} 551}\right)_{\text {int }}^{\text {rot }}$ values (see Eq. (2)) for the quiescent sample (intrinsic red galaxies) at different redshifts using the BC03 SSP models. Solid lines illustrate the MLE best fit of the $\left(m_{\mathrm{F} 365}\right.$ $\left.m_{\mathrm{F} 551}\right)_{\text {int }}^{\text {rot }}$ distribution at different redshifts. Dashed coloured lines show the upper $3 \sigma$ limit of the distributions provided by the MLE method.

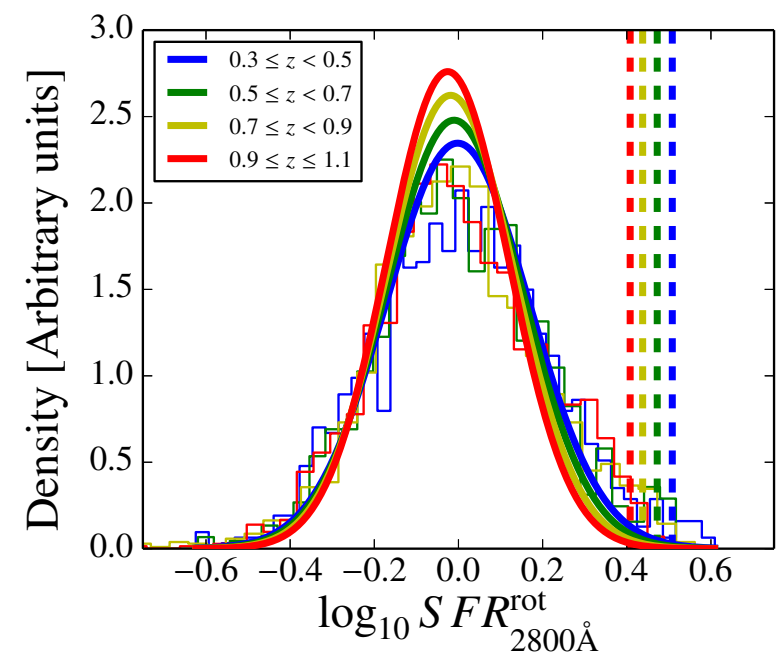

Fig. A.2. Histogram of the $\log _{10} \mathrm{SFR}_{2800 \AA}^{\text {rot }}$ values (see Eq. (11)) for the quiescent sample defined via star formation rates $\mathrm{SFR}_{2800 \AA}$ (see Eq. (8)) at different redshifts and using the BC03 SSP models. Solid lines illustrate the MLE best fit of the $\log _{10} \mathrm{SFR}_{2800 \AA}^{\text {rot }}$ distributions. Dashed coloured lines show the upper $3 \sigma$ limit of the distributions stated by the MLE method.

\section{Appendix B: Detection and removal of cool stars in the quiescent sample}

The performance of the MUFFIT module devoted to analysing stars is basically the same as for the galaxy version, but instead of using a mixture of SSP models it takes templates of stars to provide stellar parameters (effective temperature, metallicity, surface gravity, chemical composition, and extinction).

We ran the stellar version of MUFFIT with all the sources in the ALHAMBRA catalogue that present an apparent magnitude in the range $22.5 \leq m_{\mathrm{F} 814 \mathrm{~W}}<23$, using as input models the stellar library of Coelho et al. (2005) with the main grid of parameters: effective temperatures in steps of $250 \mathrm{~K}$, $3500 \leq T_{\text {eff }} \leq 7000 \mathrm{~K}$; surfaces gravities with steps of 0.5 , $0.0 \leq \log _{10} \leq 5.0$; metallicities in the range $[\mathrm{Fe} / \mathrm{H}]=-2.5$ to +0.5 ; and chemical compositions $[\alpha / \mathrm{Fe}]=0.0$ and 0.4 . In addition, we added extinction values in the range $A_{V}=0.0-3.1$ 
to the star models taking $R_{V}=3.1$ and assuming a Fitzpatrick (1999) extinction law.

After the SED fitting analysis and thanks to the Monte Carlo process performed by MUFFIT, we obtained two sets of $\chi^{2}$ values for each source. One set from the SED fitting treating the source as a galaxy and another one as a star. Therefore, the $\chi^{2}$ distribution with the lower values (the most likely set of templates, galaxy or star) will determine whether the source is a galaxy. Although this method takes advantage of the photometry in all the bands, all the sources show apparent magnitudes in the range $22.5 \leq m_{\mathrm{F} 814 \mathrm{~W}}<23$, with a reasonably low signal-to-noise ratio that makes the distinction between distributions more difficult in several cases. To solve this drawback, we carried out a Kolmogorov-Smirnov (KS) test that allowed us to determine whether the two $\chi^{2}$ distributions are different and what the probability is. We assumed that a source in the range $22.5 \leq m_{\mathrm{F} 814 \mathrm{~W}}<23$ is a star if the following criterion is satisfied: the median of $\chi^{2}$ values (from the $\chi^{2}$ distribution) is lower using star templates than SSP templates, and that the KS test additionally shows that the two distributions of $\chi^{2}$ values (star and galaxy) are not equivalent at a significance level of $1 \sigma$. Under these constraints, we found that in the redshift range $0.1 \leq z \leq 1.1$ there are 439 star candidates in the quiescent sample with $22.5 \leq m_{\mathrm{F} 814 \mathrm{~W}}<23$. Taking into account that there are 2284 quiescent galaxies in our sample at the same magnitude and redshift bin before the faint star/galaxy classification, we removed $\sim 19 \%$ of the sample at $22.5 \leq m_{\mathrm{F} 814 \mathrm{~W}}<23$.

To check whether the method developed for ALHAMBRA for removing stars in the range $22.5 \leq m_{\mathrm{F} 814 \mathrm{~W}}<23$ is reliable, we cross-matched the ALHAMBRA and COSMOS photometric catalogues (Capak et al. 2007) to build a subset of shared sources in the two surveys. Making the most of the ACS camera in COSMOS, we compared the stars or point sources detected in COSMOS and those detected by our method to estimate the degree of accuracy. There are 230 sources in common with our sample of quiescent candidates with apparent magnitudes $22.5 \leq m_{\mathrm{F} 814 \mathrm{~W}}<23$, in which we found 50 star candidates with apparent magnitudes $22.5 \leq m_{\mathrm{F} 814 \mathrm{~W}}<23$ using the ALHAMBRA photometry and MUFFIT. From the star/galaxy classification of COSMOS (Leauthaud et al. 2007) in this subsample of 230 sources, we checked that 47 (94\%) of them are classified as point sources in COSMOS. Nevertheless, there are nine stars that were not detected in the common subset with $22.5 \leq m_{\mathrm{F} 814 \mathrm{~W}}<23$ following the COSMOS classification. This indicates that about $24 \%$ of the faint cool stars should be removed. In the case where we can extrapolate these percentages from the subsample in common with COSMOS to our sample of quiescent galaxies in ALHAMBRA, we would remove $84 \%$ of faint cool stars in ALHAMBRA. Furthermore, we would expect a contamination of around $4 \%$ of the stars in our sample of quiescent galaxies at $22.5 \leq m_{\mathrm{F} 814 \mathrm{~W}}<23$.

\section{Appendix C: Stellar mass completeness determination}

Our aim is to develop a method for determining and parametrising the stellar mass completeness of the ALHAMBRA galaxies. This method must be applicable to the sample of quiescent galaxies in this work and also easily calculable for any completeness level $C$.

Our methodology is based on taking advantage of stellar mass functions, $\Phi(M)$, from deeper surveys. In particular, we took the stellar mass functions from the COSMOS survey for quiescent galaxies (Ilbert et al. 2010). We tried to measure how the low-mass end of the ALHAMBRA sample differs from the one derived from this much deeper survey, assuming that the differences are dominated by the stellar mass completeness. To include the impact of stellar mass completeness in stellar mass functions, we followed a process similar to the work described in Sandage et al. (1979), which relies on a likelihood maximisation. This likelihood, $\mathcal{L}$, encompasses the probability of observing a galaxy accounting for both the selection and observational effects. Formally,

$\ln \mathcal{L}=\sum_{i=1}^{N_{\mathrm{g}}} \frac{\Phi\left(M_{i}\right) f\left(M_{i}\right)}{\int_{M_{i, \min }}^{M_{i, \mathrm{ax}}} \Phi\left(M^{\prime}\right) f\left(M^{\prime}\right) \mathrm{d} M^{\prime}}$,

where $N_{\mathrm{g}}$ is the number of galaxies in the subsample, $f\left(M_{i}\right)$ is the stellar mass completeness for a galaxy with stellar mass $M_{i}$ at certain redshift, $M_{i, \min }$ and $M_{i, \max }$ are respectively the minimum and maximum stellar masses at the redshift in which the $i$ th galaxy resides, and $\Phi(M)$ the stellar mass function characterised by a Schechter-like function (Schechter 1976) through three parameters $\left(\alpha, \mathcal{M}_{*}\right.$, and the normalisation $\Phi^{*}$; see Fontana et al. 2004; Pérez-González et al. 2008; Vergani et al. 2008; Ilbert et al. 2010, 2013) expressed as

$\Phi(M) \mathrm{d} M=\Phi^{*}\left(\frac{M}{\mathcal{M}_{*}}\right)^{\alpha} \exp \left(-\frac{M}{\mathcal{M}_{*}}\right) \mathrm{d}\left(\frac{M}{\mathcal{M}_{*}}\right)$.

As in Sandage et al. (1979), we used a Fermi-Dirac distribution function, $f_{\mathrm{FD}}$, to parametrise the stellar mass completeness, formally expressed as

$f_{\mathrm{FD}}\left(z, M_{\star}\right)=\frac{1}{\exp \left[\left(M_{F}(z)-\log _{10} M_{\star}\right) / \Delta_{F}(z)\right]+1}$,

where $M_{F}(z)$ is the stellar mass value (in dex) for which the completeness reaches $50 \%(C=0.5)$ and $\Delta_{F}(z)$ is related to the decrease rate on the number of galaxies. It should be noted that both $M_{F}(z)$ and $\Delta_{F}(z)$ are redshift dependent. The stellar mass value limit for a given completeness level and redshift, $M_{C}(z)$, can be easily derived from Eq. (C.3) as

$\log _{10} M_{C}(z)=M_{F}(z)+\Delta_{F}(z) \ln \left[\frac{C}{1-C}\right]$.

After fixing both $\alpha$ and $\mathcal{M}_{*}$ to the values obtained by Ilbert et al. (2010, the term $\Phi^{*}$ is not relevant because it is cancelled in Eq. (C.1)), the maximisation of Eq. (C.1) provides us $M_{F}$ and $\Delta_{F}$. For the maximisation of the likelihood, we used again the emcee algorithm, for which we set limiting values of $5 \leq M_{F}(z) \leq 14$ and $0.02 \leq \Delta_{F}(z)<2$.

As a result, we show in Table 4 the values $M_{F}$ and $\Delta_{F}$ that maximise Eq. (C.1), and also show the stellar mass limits for different completeness levels for the quiescent galaxies in ALHAMBRA down to $m_{\mathrm{F} 814 \mathrm{~W}}=23$. These values were obtained for BC03 SSP models, that is, the same model set used by Ilbert et al. (2010) for the COSMOS survey. We checked to see if there were systematic discrepancies between the stellar masses obtained using EMILES and those obtained using BC03 SSP models. Systematically, stellar masses for quiescent galaxies are about 0.15 dex $(0.11 \mathrm{dex})$ more massive using the EMILES SSP models with BaSTI (Padova00) isochrones in comparison with the BC03 ones. For EMILES, the completeness is reproduced well when we add this systematic difference ( 0.15 and $0.11 \mathrm{dex})$ in $M_{F}$ without altering $\Delta_{F}$. As a sanity check, we compared our predictions of mass limits at $C=0.95$ with those obtained following the methodology proposed by Pozzetti et al. (2010). We found good agreement between the two methods, with mean differences of $\sim 0.03$ dex at $0.1<z<1.1$. 


\section{Appendix D: Stellar population properties of quiescent galaxies using EMILES SSP models}

As detailed in Sect. 3, we assessed potential systematics from the use of different population synthesis models by building sets of composite stellar population models through two separate sets of SSP models. Below, we illustrate some of the results presented and discussed in Sects. 4-7, but using the EMILES SSP models for BaSTI (Figs. D.1-D.8) and Padova00 isochrones (Figs. D.9D.16).
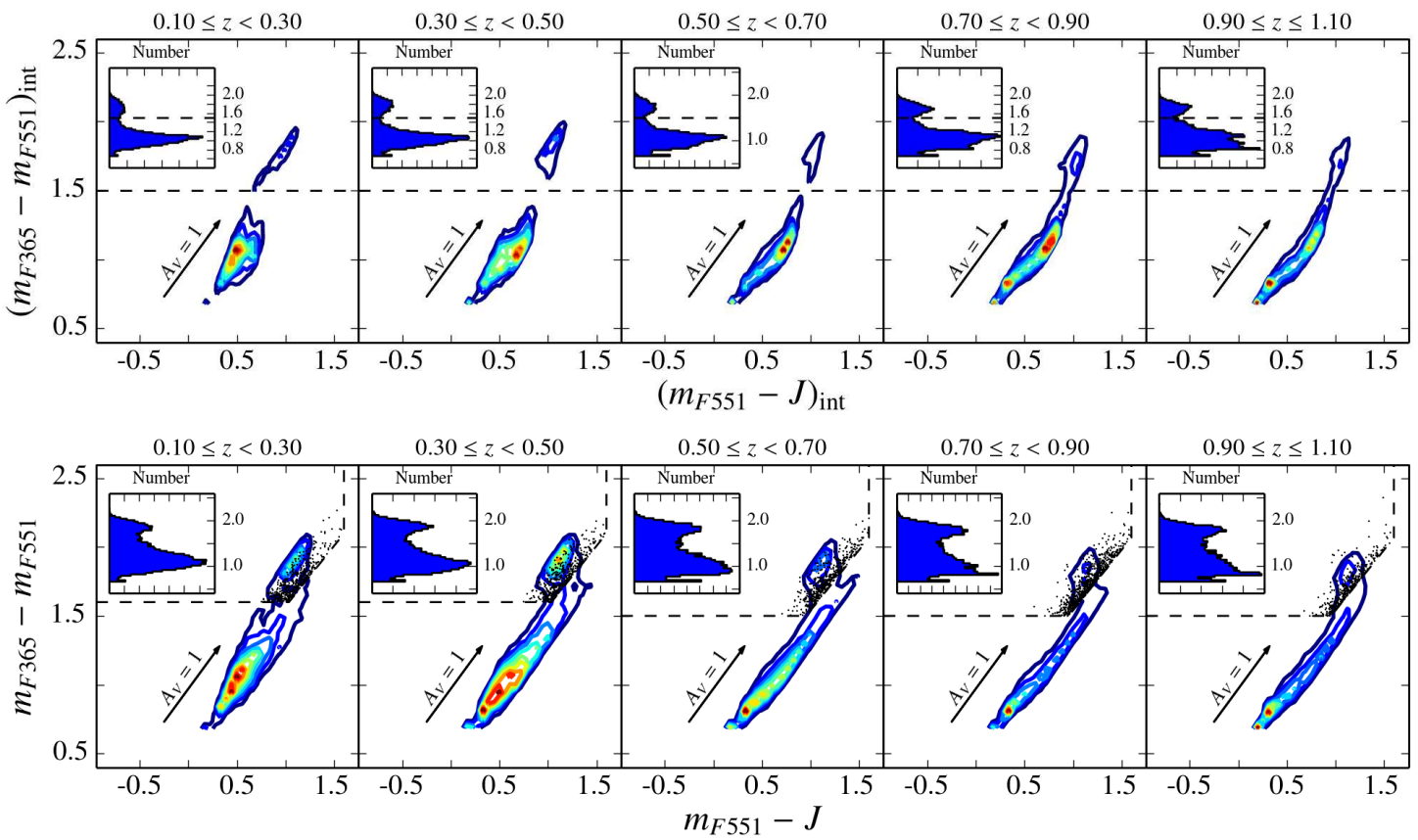

Fig. D.1. Density surface and distribution of rest-frame colours for galaxies from the ALHAMBRA survey obtained using EMILES+BaSTI SSP models. Top: rest-frame intrinsic colours $\left(m_{\mathrm{F} 551}-J\right)_{\mathrm{int}}(X$-axis $)$ and $\left(m_{\mathrm{F} 365}-m_{\mathrm{F} 551}\right)_{\text {int }}(Y$-axis $)$ after correcting for extinction at different redshifts. Bottom: rest-frame colours without removing dust effects. Redder (bluer) density-curve colours are related to higher (lower) densities. Inner panels, histograms of the intrinsic (top) and observed (bottom) rest-frame colour $m_{\mathrm{F} 365}-m_{\mathrm{F} 551}$. Dashed lines in the top panels illustrate our limiting value $\left(m_{\mathrm{F} 365}-m_{\mathrm{F} 551}\right)_{\mathrm{int}}=1.5$ for quiescent galaxies, and in the bottom panels the quiescent $U V J$ sample defined by Moresco et al. (2013, Eq. (1)). Black dots are galaxies labelled as quiescent by the $U V J$ criteria of Moresco et al. (2013) that lie in the star-forming region after removing extinction effects. We illustrate the colour variations due to a reddening of $A_{V}=1$ (black arrow), assuming the extinction law of Fitzpatrick (1999).

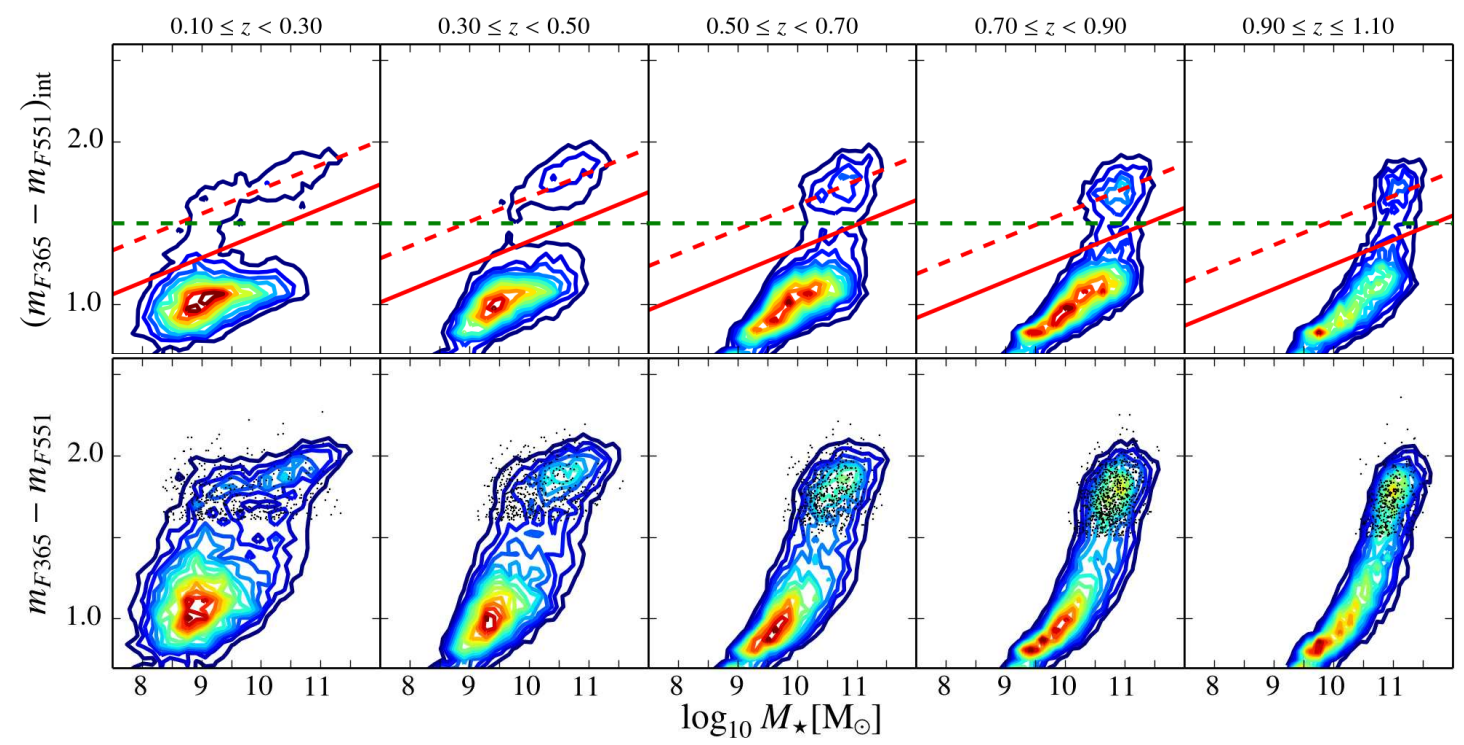

Fig. D.2. Density surface and distribution of stellar mass vs. rest-frame colour $\left(m_{\mathrm{F} 365}-m_{\mathrm{F} 551}\right)$ for galaxies from the ALHAMBRA survey using EMILES+BaSTI SSP models. Top: rest-frame intrinsic colour $\left(m_{\mathrm{F} 365}-m_{\mathrm{F} 551}\right)_{\text {int }}(Y$-axis $)$ after correcting for extinction at different redshift. Bottom: rest-frame colours without removing dust effects. Redder (bluer) density-curve colours are related to high (low) densities. Dashed green lines in the top panels illustrate the limiting value $\left(m_{\mathrm{F} 365}-m_{\mathrm{F} 551}\right)_{\text {int }}=1.5$ used for selecting quiescent galaxies in Sect. 4.1. Dashed and solid red lines show the fit to the quiescent sequence, $\left(m_{\mathrm{F} 365}-m_{\mathrm{F} 551}\right)_{\mathrm{int}}^{\mathrm{Q}}$, and the limiting intrinsic colours, $\left(m_{\mathrm{F} 365}-m_{\mathrm{F} 551}\right)_{\text {int }}^{\lim }$, values of quiescent galaxies respectively (see details in Sect. 4.2). Black dots in the bottom panels are galaxies labelled as quiescent with the $U V J$ criteria of Moresco et al. (2013) that lie in the star-forming region after removing extinction effects. 


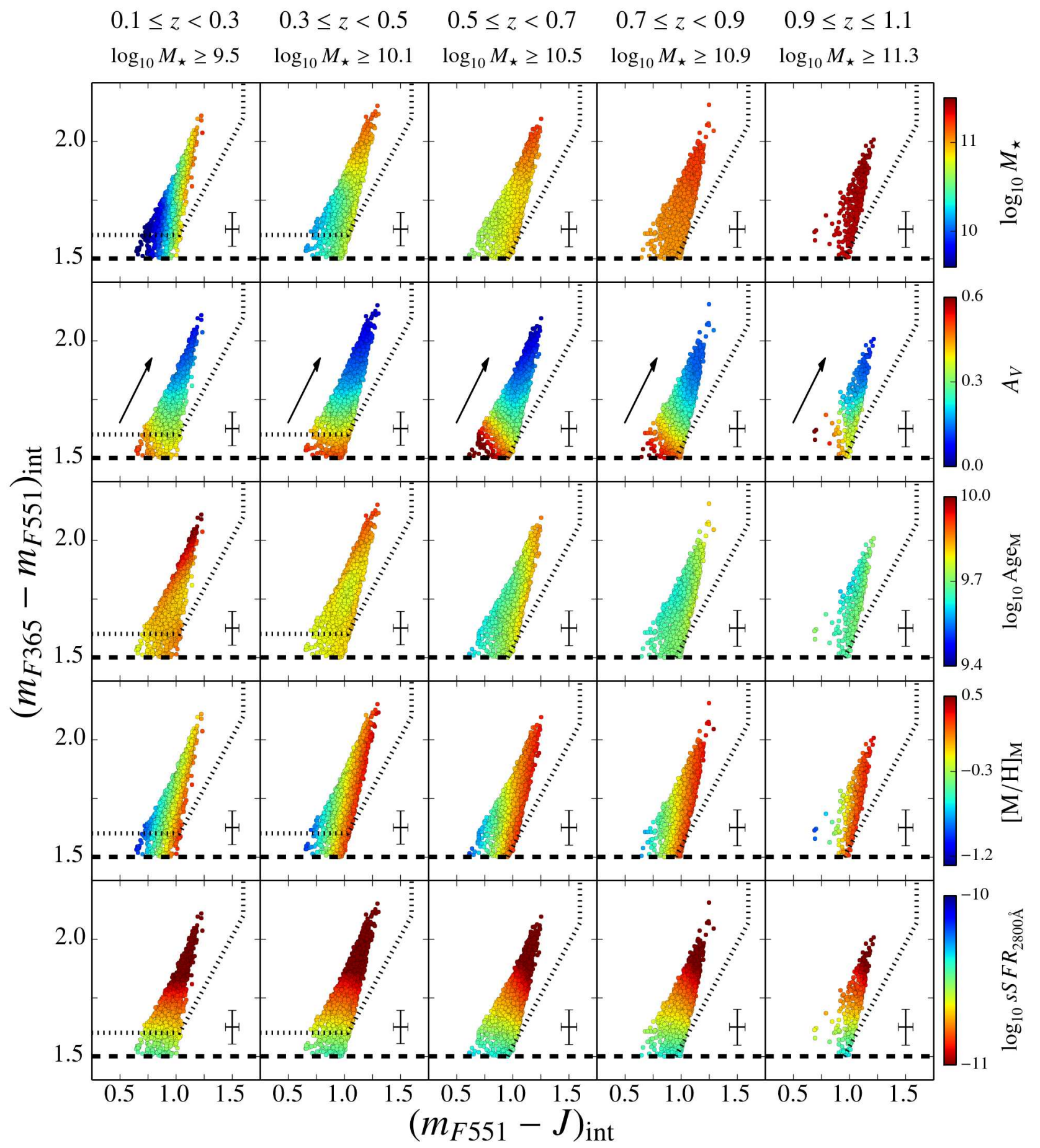

Fig. D.3. Stellar population parameters in the rest-frame $U V J$ diagram. At different redshift bins, we present the intrinsic colours $\left(m_{\mathrm{F} 551}-J\right)_{\text {int }}(X-$ axis) and $\left(m_{\mathrm{F} 365}-m_{\mathrm{F} 551}\right)_{\text {int }}(Y$-axis $)$ after correcting for extinction for the mass complete sample of quiescent galaxies (see stellar mass completeness at the top). The different stellar population parameters are colour-coded as a function of their values and obtained using EMILES+BaSTI SSP models (see the colour bars to the right of each row). From top to bottom: stellar mass, extinction, mass-weighted age and metallicity, and specific star formation rate. All the parameters were spatially averaged through a LOESS method. Black crosses illustrate the median uncertainties in both $\left(m_{\mathrm{F} 551}-J\right)_{\text {int }}$ and $\left(m_{\mathrm{F} 365}-m_{\mathrm{F} 551}\right)_{\text {int }}$ intrinsic colours. The dotted black line encloses the rest-frame colour ranges assumed for selecting quiescent galaxies in Moresco et al. (2013, see Eq. (1)), while the dashed line illustrates our colour limit for selecting quiescent galaxies $\left(m_{\mathrm{F} 365}-m_{\mathrm{F} 551}\right)_{\mathrm{int}}>$ 1.5. We illustrate the colour variations owing to a reddening of $A_{V}=0.5$ (black arrow), assuming the extinction law of Fitzpatrick (1999). 


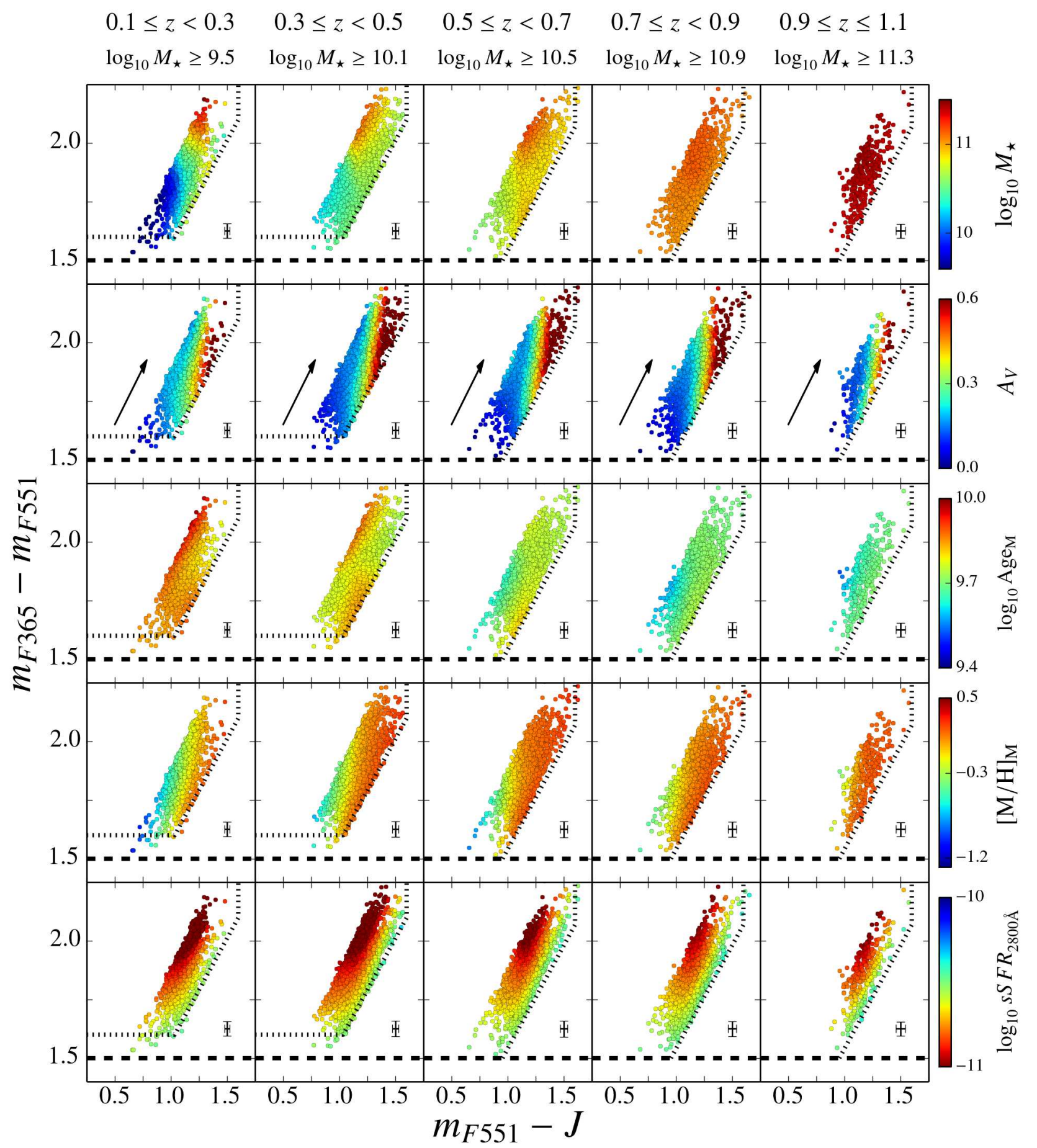

Fig. D.4. Same as Fig. D.3, but for the rest-frame colours $m_{\mathrm{F} 551}-J$ (X-axis) and $m_{\mathrm{F} 365}-m_{\mathrm{F} 551}(Y$-axis), meaning non-dust corrected colours. 


\section{$0.1 \leq z<0.3 \quad 0.3 \leq z<0.5 \quad 0.5 \leq z<0.7 \quad 0.7 \leq z<0.9 \quad 0.9 \leq z \leq 1.1$}

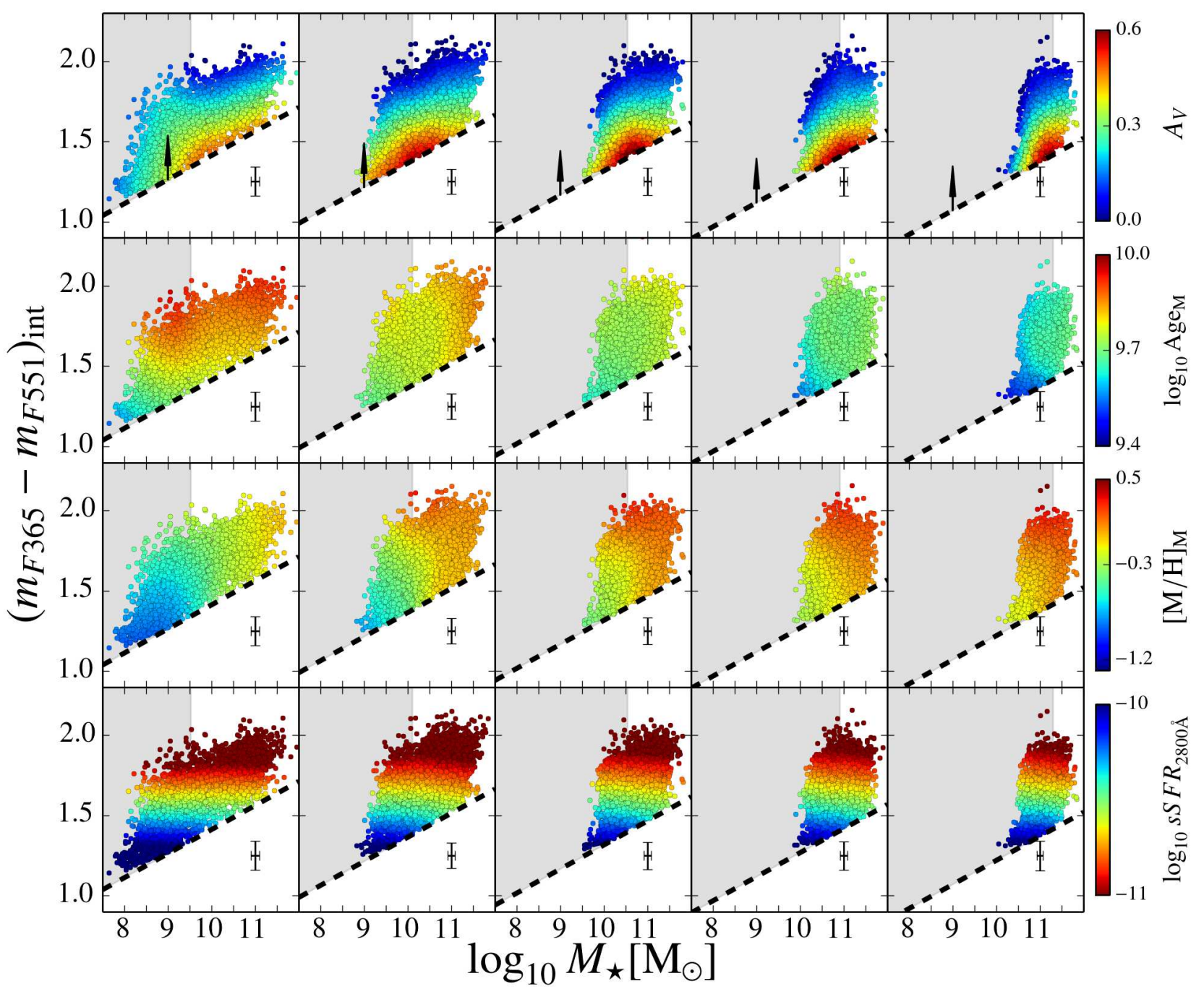

Fig. D.5. Stellar population parameters in the rest-frame stellar mass-colour diagram. At different redshift bins, we present the stellar mass $(X$ axis) and intrinsic colour $\left(m_{\mathrm{F} 365}-m_{\mathrm{F} 551}\right)_{\text {int }}(Y$-axis) after correcting for extinction. The stellar population parameters are colour-coded according to their values using EMILES+BaSTI SSP models (see colour bars). From top to bottom: extinction, mass-weighted age and metallicity, and specific star formation rate. All the parameters were spatially averaged through a LOESS method. Black crosses illustrate the median uncertainties in both stellar mass and $\left(m_{\mathrm{F} 365}-m_{\mathrm{F} 551}\right)_{\text {int }}$ intrinsic colour. The dashed line illustrates the colour limit for selecting quiescent galaxies in the MCDE, see Eq. (3) and Table 3, for this work. The shaded regions show the stellar mass range in which our quiescent sample is not complete in stellar mass. We illustrate the colour variations owing to a reddening of $A_{V}=0.5$ (black arrow), assuming the extinction law of Fitzpatrick (1999). 


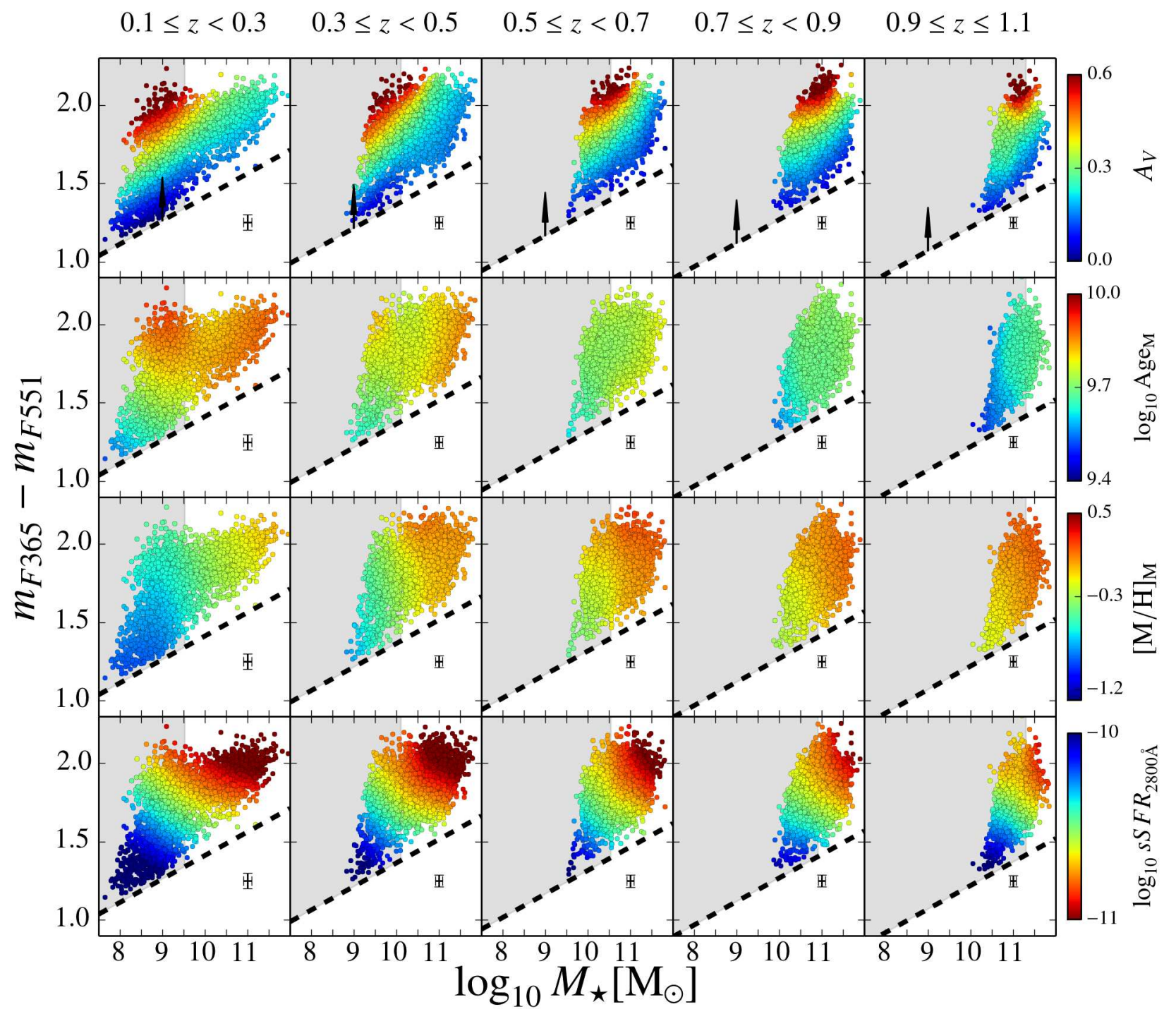

Fig. D.6. Same as Fig. D.5, but for the rest-frame colour $m_{\mathrm{F} 365}-m_{\mathrm{F} 551}(X$-axis, non-dust corrected colour).

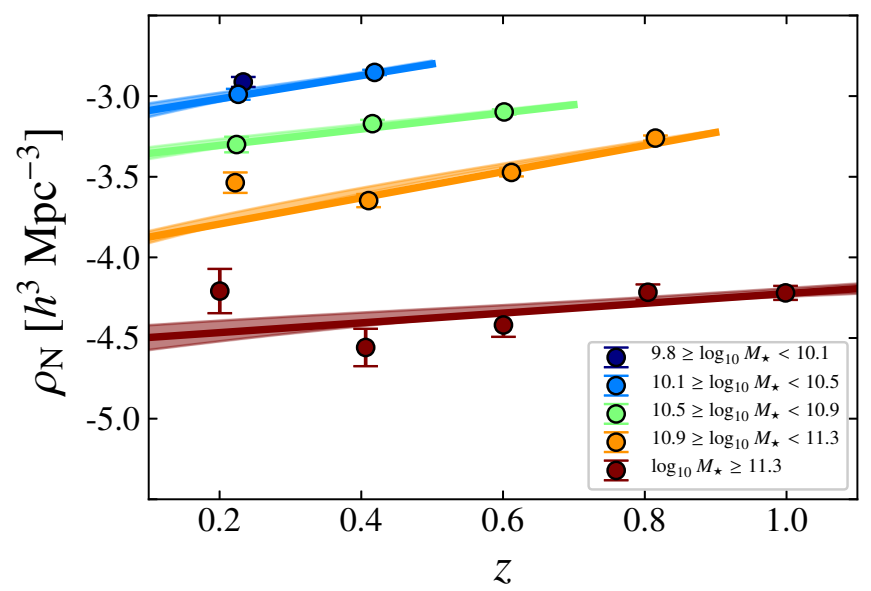

Fig. D.7. Evolution of co-moving number density, $\rho_{\mathrm{N}}(z)$, of green valley galaxies with redshift ( $X$-axis) at different stellar mass bins (see inset) using EMILES+BaSTI SSP models. Coloured markers illustrate co-moving number densities at redshift bins and vertical bars are their uncertainties. Solid lines illustrate the best fit to Eq. (13), whereas the shaded regions show the $1 \sigma$ uncertainty of the fit. 


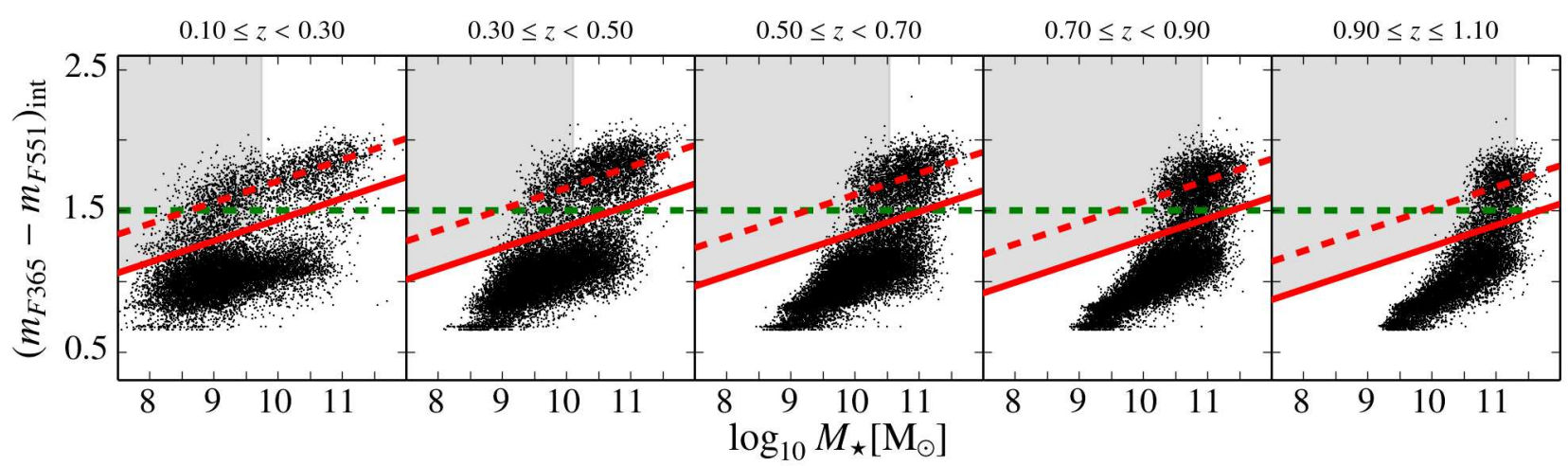

Fig. D.8. Galaxies from the ALHAMBRA survey (black dots) in the rest-frame stellar mass-colour diagram corrected for extinction (MCDE) at different redshift bins. The shaded region illustrates the stellar mass range in which our sample of quiescent galaxies is not complete in stellar mass (see Sect. 4.5) at each redshift bin. The dashed green line shows the limiting value $\left(m_{\mathrm{F} 365}-m_{\mathrm{F} 551}\right)_{\text {int }}=1.5$ used to select quiescent galaxies in Sect. 4.1. The dashed red line exhibits the main sequence of quiescent galaxies in the MCDE, see Eq. (3) and Table 3, and the solid line is the limiting colour value used to select quiescent galaxies in this diagram, see Eq. (3) and Table 3. The stellar population predictions were obtained through EMILES+BaSTI SSP models.
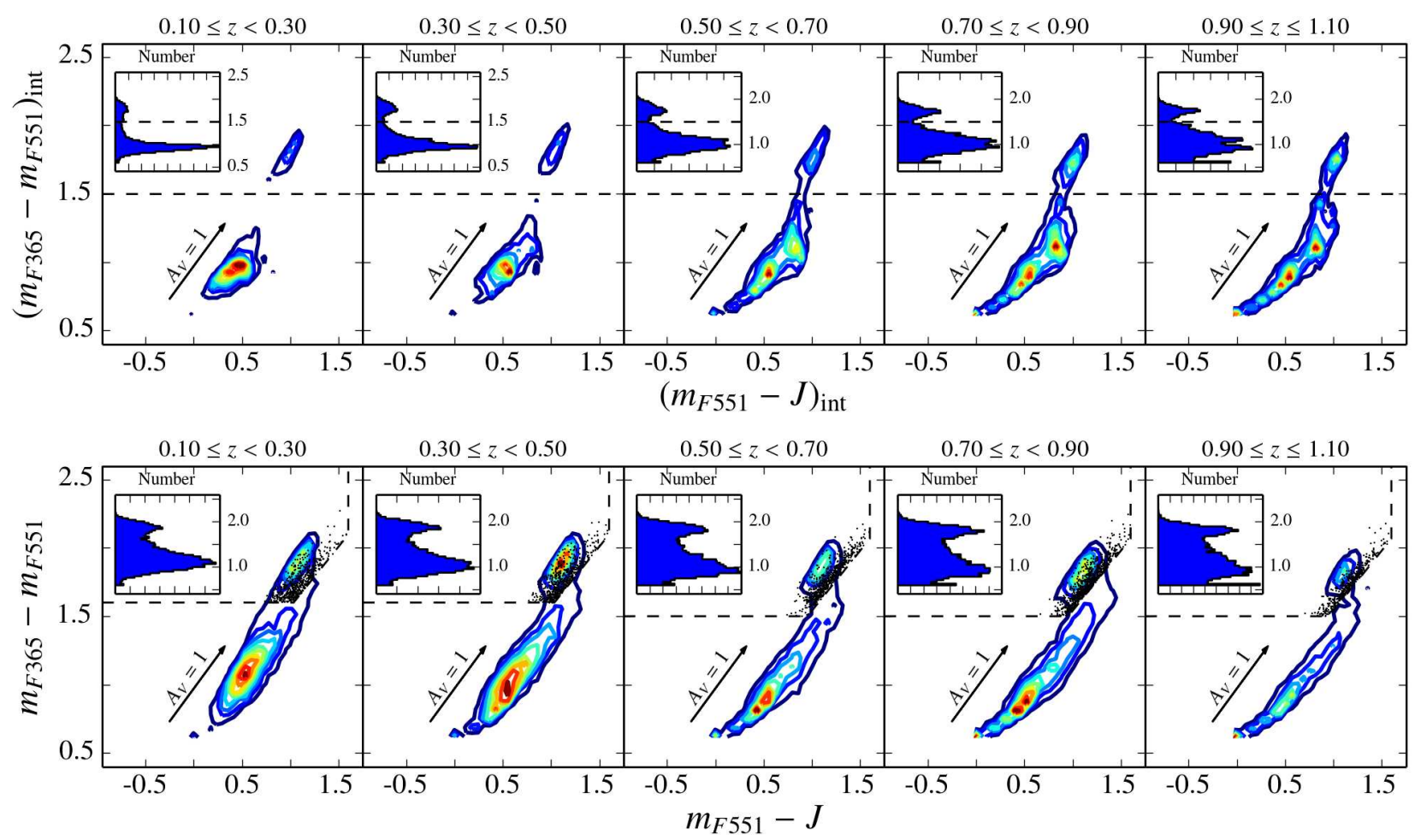

Fig. D.9. Same as Fig. D.1, but for Padova00 isochrones. 


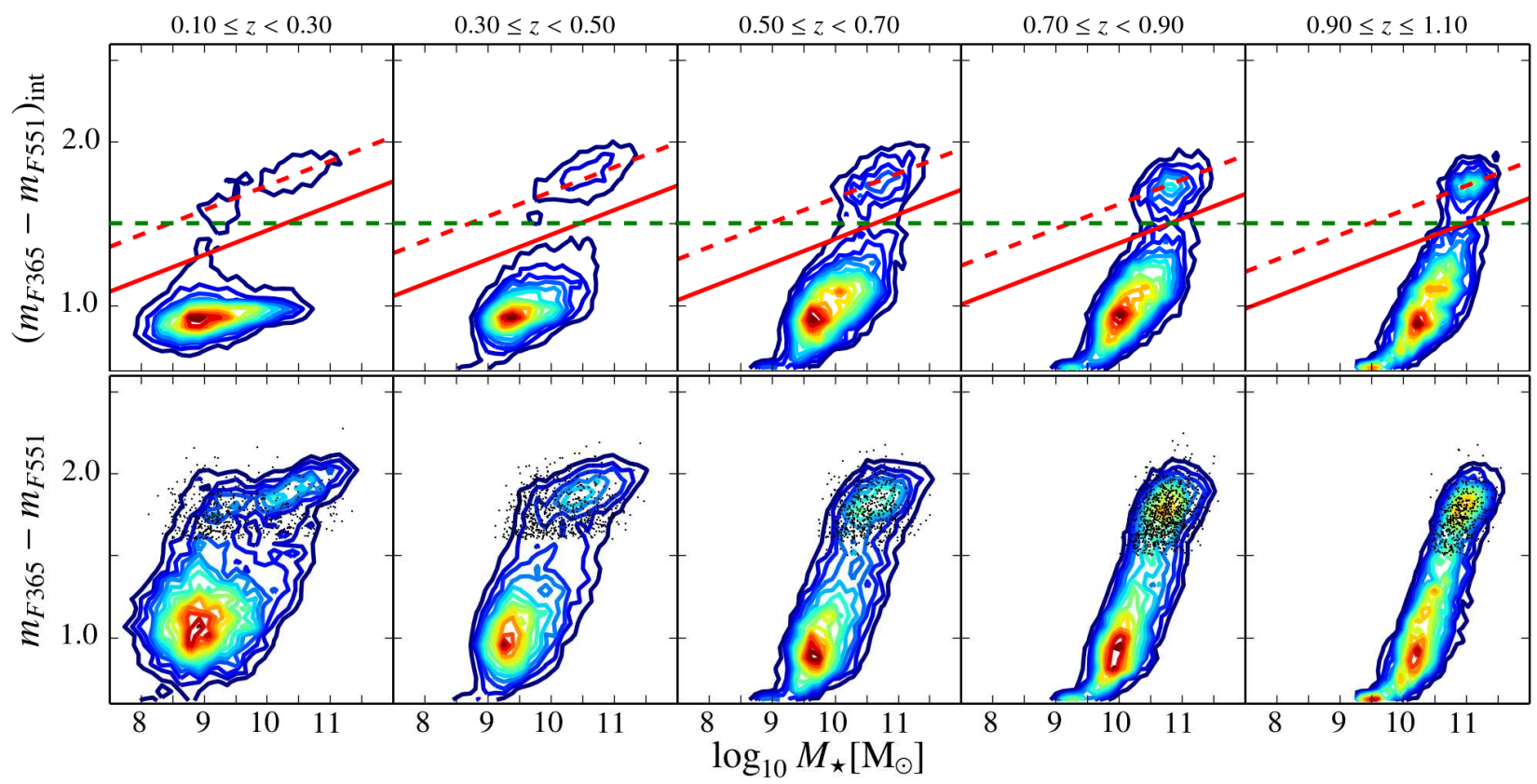

Fig. D.10. Same as Fig. D.2, but using Padova00 isochrones. 
L. A. Díaz-García et al.: Stellar populations of galaxies within diagrams involving colours

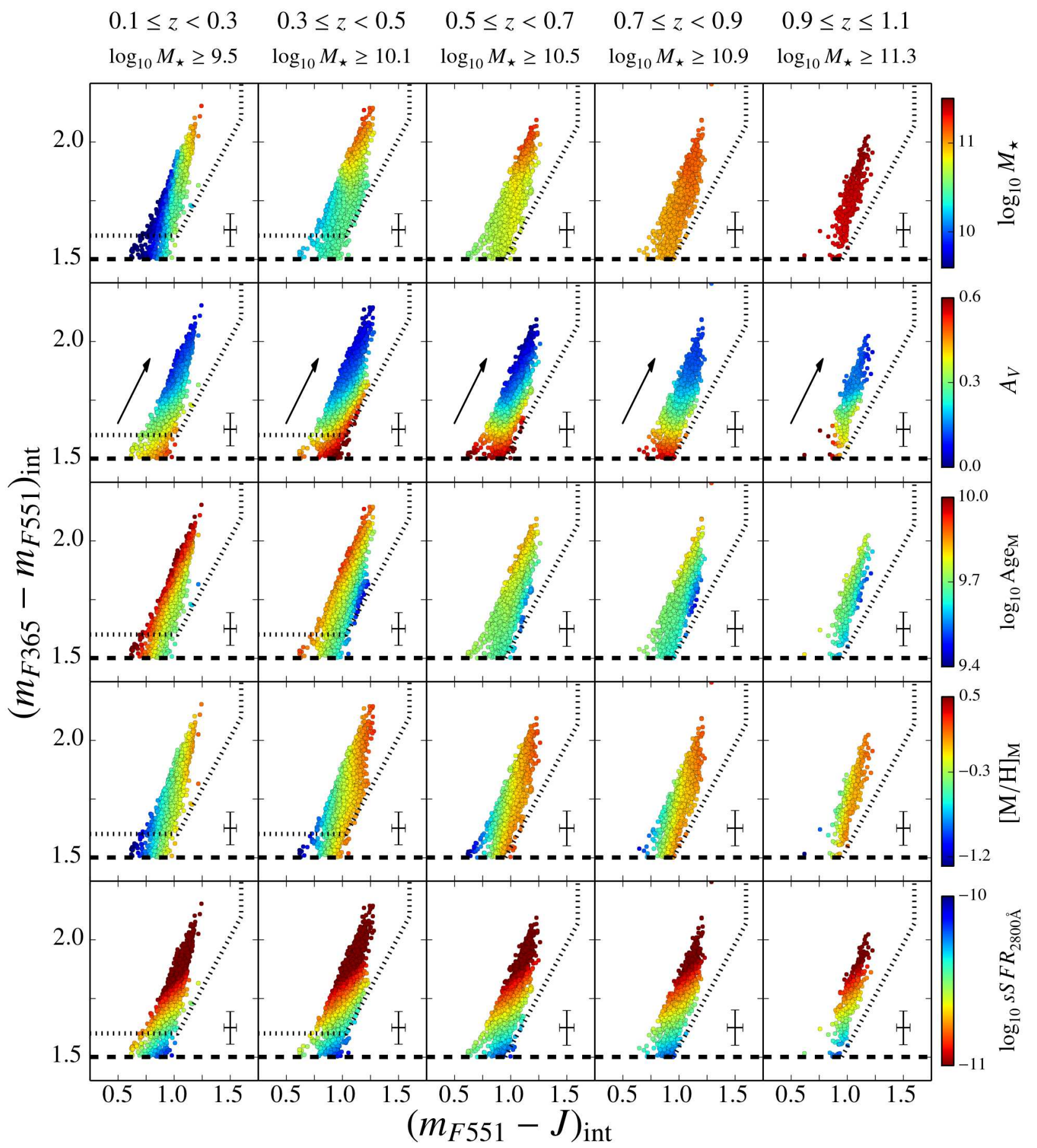

Fig. D.11. Same as Fig. D.3, but using EMILES+Padova00 SSP models. 


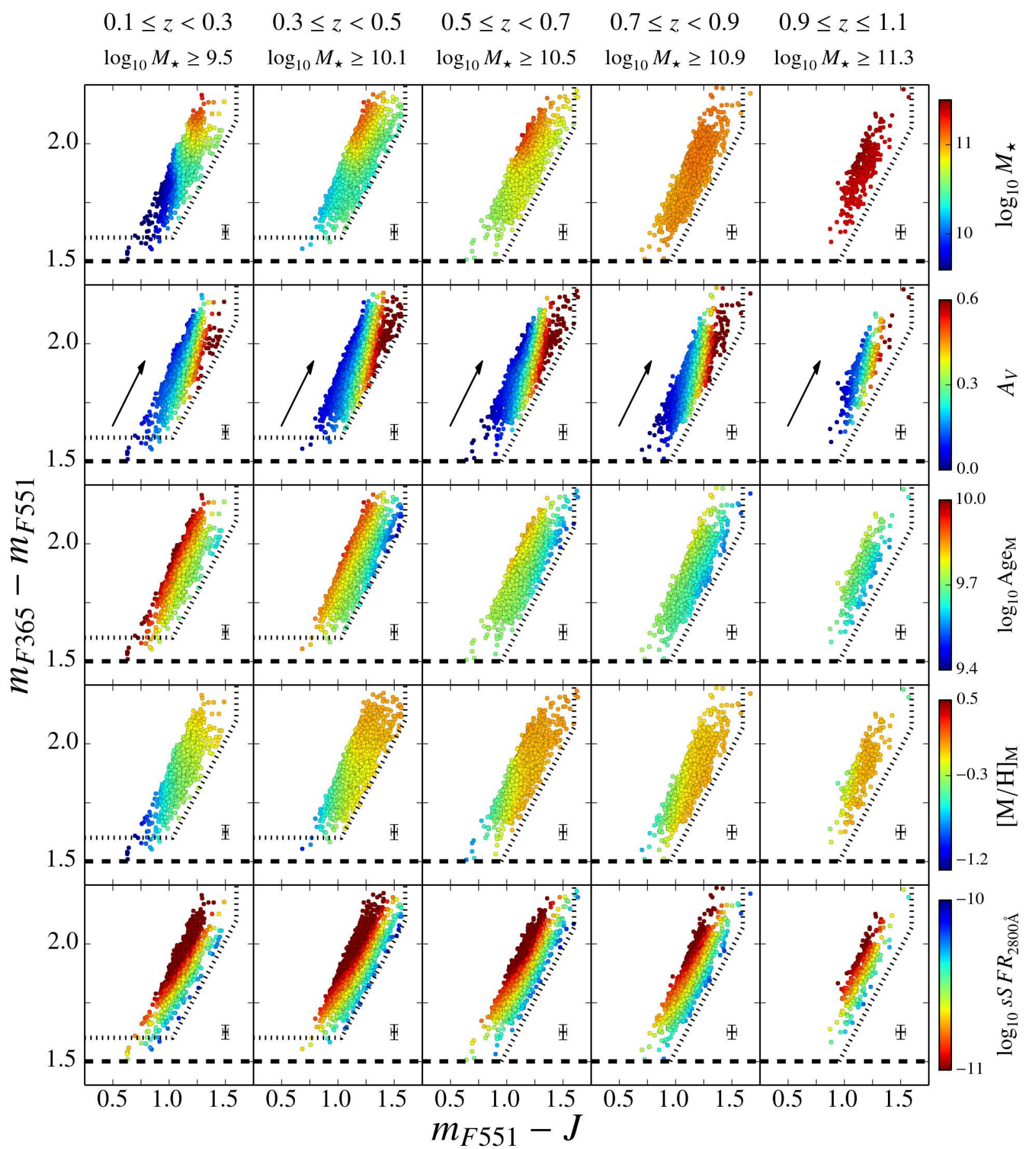

Fig. D.12. Same as Fig. D.4, but using EMILES+Padova00 SSP models. 
L. A. Díaz-García et al.: Stellar populations of galaxies within diagrams involving colours

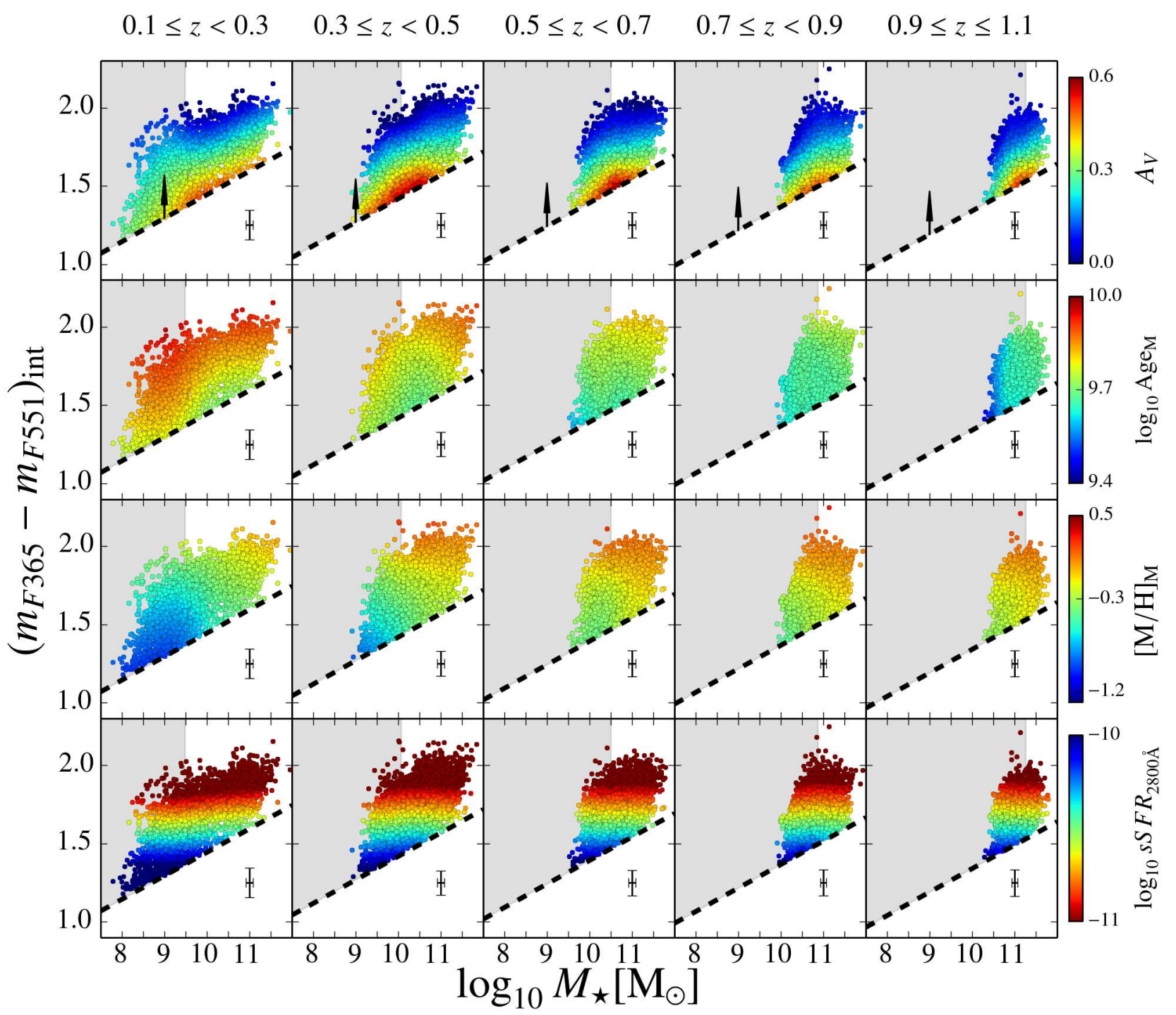

Fig. D.13. Same as Fig. D.5, but using EMILES+Padova00 SSP models. 


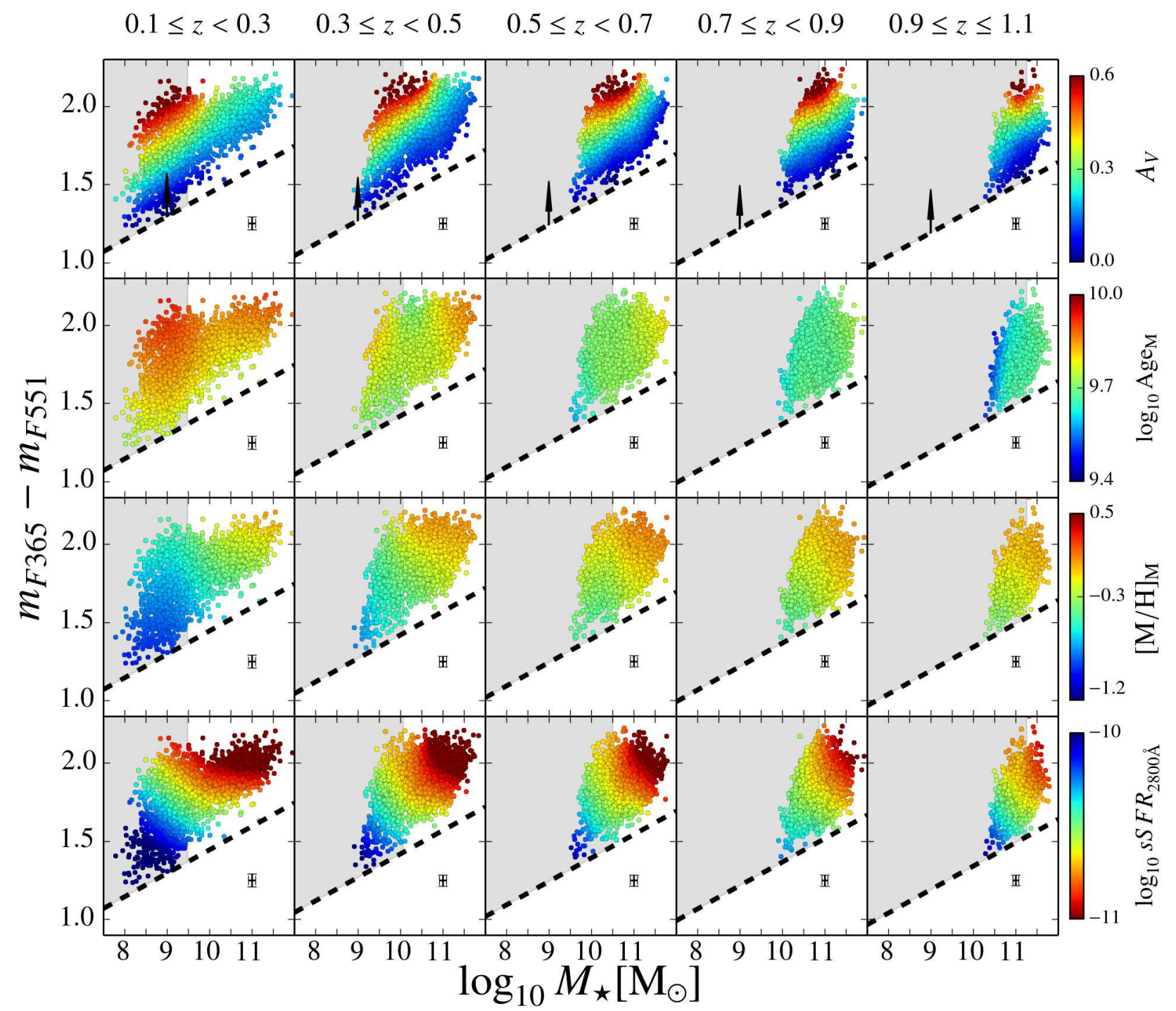

Fig. D.14. Same as Fig. D.6, but using EMILES+Padova00 SSP models.

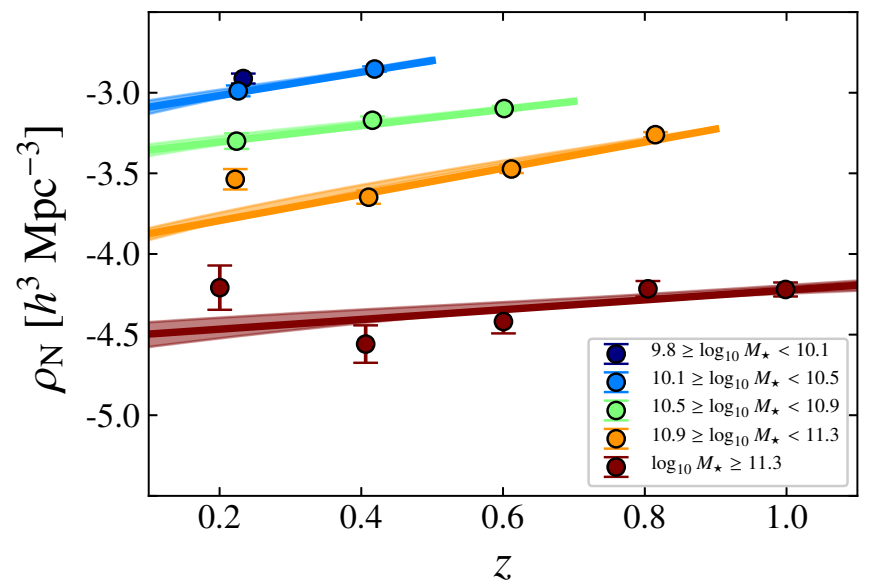

Fig. D.15. Same as Fig. D.7, but using EMILES+Padova00 SSP models. 
L. A. Díaz-García et al.: Stellar populations of galaxies within diagrams involving colours

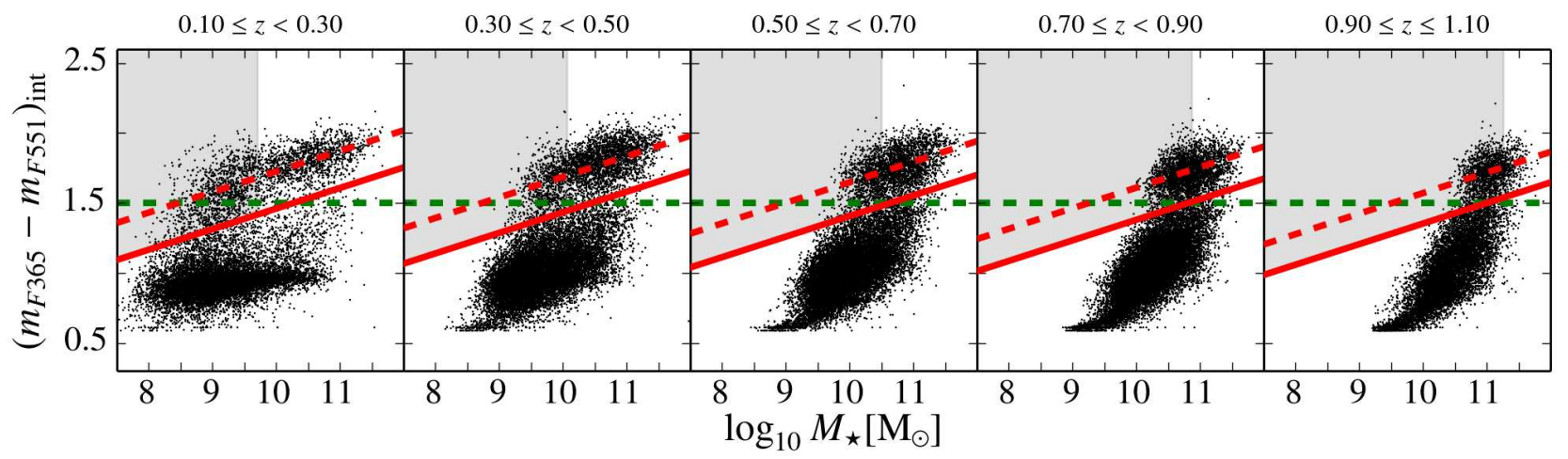

Fig. D.16. Same as Fig. D.8, but using EMILES+Padova00 SSP models. 